\title{
Regular cell complexes in total positivity
}

\section{Patricia Hersh}

Received: 3 May 2011 / Accepted: 5 July 2013 / Published online: 3 August 2013

(C) The Author(s) 2013. This article is published with open access at Springerlink.com

\begin{abstract}
Fomin and Shapiro conjectured that the link of the identity in the Bruhat stratification of the totally nonnegative real part of the unipotent radical of a Borel subgroup in a semisimple, simply connected algebraic group defined and split over $\mathbb{R}$ is a regular CW complex homeomorphic to a ball. The main result of this paper is a proof of this conjecture. This completes the solution of the question of Bernstein of identifying regular $\mathrm{CW}$ complexes arising naturally from representation theory having the (lower) intervals of Bruhat order as their closure posets. A key ingredient is a new criterion for determining whether a finite $\mathrm{CW}$ complex is regular with respect to a choice of characteristic maps; it most naturally applies to images of maps from regular CW complexes and is based on an interplay of combinatorics of the closure poset with codimension one topology.
\end{abstract}

\section{Introduction}

In this paper, the following conjecture of Sergey Fomin and Michael Shapiro from [11] is proven.

Conjecture 1.1 Let $Y$ be the link of the identity in the totally nonnegative real part of the unipotent radical of a Borel subgroup $B$ in a semisimple, simply connected algebraic group defined and split over $\mathbb{R}$. Let $B_{u}=B^{-} u B^{-}$for $u$ in the Weyl group $W$. Then the stratification of $Y$ into Bruhat cells $Y \cap B_{u}$ is a regular $C W$ decomposition. Moreover, for each $w \in W, Y_{w}=\bigcup_{u \leq w} Y \cap B_{u}$

P. Hersh ( $\square)$

North Carolina State University, Raleigh, NC 27695-8205, USA

e-mail: plhersh@ncsu.edu 
is a regular CW complex homeomorphic to a ball, as is the link of each of its cells.

This is done in Theorem 6.1. The result includes, for instance, the special case that the link of the identity in the Bruhat stratification of the space of upper triangular matrices with 1's on the diagonal whose minors are all nonnegative is a regular CW complex homeomorphic to a ball; more specifically, this is the collection of upper triangular, totally nonnegative matrices with 1's on the diagonal and entries immediately above the diagonal summing to a positive constant, stratified according to which minors are strictly positive and which are 0 . The poset (partially ordered set) of closure relations is Bruhat order.

This positively answers the question below regarding synthetic Schubert varieties which appeared in a paper of Björner (see [4]), but was actually posed by Joseph Bernstein (personal communication, Anders Björner):

Question 1.2 It would be of considerable interest to know which $(\mathrm{CW})$ posets can be reasonably interpreted as face posets of cellular decompositions of complex algebraic varieties, and whether there is a synthetic construction for doing so. In particular, can 'synthetic Schubert varieties' be naturally associated with the (lower) Bruhat intervals of any Coxeter group.

Results of Björner [4] combine with results of Björner and Wachs [6] to imply that each interval of Bruhat order is the closure poset of a regular CW complex. This is what led to Question 1.2. Fomin and Shapiro proved in [11] that the closure relations for $Y_{w}=\bigcup_{u \leq w} Y \cap B_{u}$ are exactly those of the lower interval $(1, w)$ in Bruhat order, they obtained substantial homological results regarding this space (especially in type A), and they formulated Conjecture 1.1. Lusztig interpreted $Y_{w}$ in [20] as the image of a continuous map $f_{\left(i_{1}, \ldots, i_{d}\right)}$ given by a reduced word $\left(i_{1}, \ldots, i_{d}\right)$ for $w$, which is the viewpoint we will take as well. The tools we develop in order to prove Conjecture 1.1 give a new approach to the general question of how to prove that the image of a map from a polytope (or slightly more general regular CW complex) which restricts to a homeomorphism on the interior but not necessarily on the boundary is a regular CW complex homeomorphic to a ball.

Motivation for studying these totally nonnegative parts $Y_{w}$ of varieties comes from a relationship observed by Lusztig to his theory of canonical bases. The change of coordinates map resulting from applying a braid move to a reduced word specifying a canonical basis is a tropicalized version of the corresponding change of coordinates for a totally nonnegative variety (cf. [20-22]). Trying to understand such changes of coordinates was also an inspiration for the theory of cluster algebras (see [2,13]). Our topological approach seems to give a somewhat new perspective on these changes of coordinates. The explicit collapsing maps we develop and use later will give quite 
explicit information about fibers of a map whose inverse has been the subject of considerable study (see e.g. [13] and the preprint [25]). For instance, we deduce connectedness of fibers indirectly, using our Theorem 1.3 below.

Our starting point was the following new criterion for determining whether a finite CW complex is regular with respect to a choice of characteristic maps. See Sect. 2 for a review of the requisite definitions. This result below gave us a route through which to approach Conjecture 1.1.

Conditions 1 and 2 below imply that the closure poset is graded by cell dimension, ensuring that the subsequent conditions make sense. Condition 3 is a combinatorial condition which (together with Condition 2) enables injectivity of attaching maps to be proven by an induction on difference in dimensions. Condition 4 gives the base case for this induction. Notably absent is a more general requirement of injectivity for the attaching maps.

Theorem 1.3 Let $K$ be a finite $C W$ complex with characteristic maps $f_{\alpha}$ : $B^{\operatorname{dim} e_{\alpha}} \rightarrow \overline{e_{\alpha}}$. Then $K$ is regular with respect to these characteristic maps $\left\{f_{\alpha}\right\}$ if and only if the following conditions hold:

(1) For each $\alpha, f_{\alpha}\left(B^{\operatorname{dim} e_{\alpha}}\right)$ is a union of open cells.

(2) For each $f_{\alpha}$, the preimages of the open cells of dimension $\operatorname{dim} e_{\alpha}-1$ form a dense subset of the boundary of $B^{\operatorname{dim} e_{\alpha}}$.

(3) The closure poset of $K$ is thin, i.e., each closed interval $[u, v]$ with $r k(v)-r k(u)=2$ has exactly four elements. Additionally, each open interval $(u, v)$ with $r k(v)-r k(u)>2$ is connected.

(4) For each $\alpha$, the restriction of $f_{\alpha}$ to the preimages of the open cells of dimension exactly one less than $e_{\alpha}$ is an injection.

(5) For each $e_{\sigma} \subseteq \overline{e_{\alpha}}, f_{\sigma}$ factors as an embedding $\iota: B^{\operatorname{dim} e_{\sigma}} \rightarrow B^{\operatorname{dim} e_{\alpha}}$ followed by $f_{\alpha}$.

Theorem 1.3 is proven in Sect. 3. Examples are also given in Sect. 3 demonstrating that each of Conditions 2, 3, 4, and 5 is not redundant. Condition 5 makes Theorem 1.3 seem likely to be applicable primarily to images of maps from regular CW complexes, which is indeed how we will use Theorem 1.3.

Our proof of Conjecture 1.1 relies critically upon the fact that condition 4, codimension one injectivity, follows easily in our setting from the exchange axiom for Coxeter groups (which is reviewed in Sect. 2). The analogous Coxeter-theoretic statement is not true in higher codimensions, seemingly demonstrating the efficacy of Theorem 1.3. One reason for interest in proving stratified spaces to be regular CW complexes is the appealing feature of regular CW complexes that their topological structure (homeomorphism type) is determined by the combinatorics of their posets of closure relations.

The proof of Conjecture 1.1 also involves the development of a combinatorial topological toolkit for performing a series of collapses on a convex 
polytope (which in our case is a simplex) in a manner that preserves regularity and homeomorphism type at each step. Each collapse reduces the number of cells by eliminating some cells and identifying other cells with each other. These collapses that we introduce in Theorem 4.21 (and its extension in Corollary 4.24) are much in the spirit of elementary collapses, but with a tighter control on the maps which enables us to preserve not just homotopy type but homeomorphism type as well. They resemble the process of Bing shrinking (cf. [3]) in that we extend collapsing maps across collars by giving paths of homeomorphisms deforming each collapsing map to the identity map. We specifically needed to develop a class of collapses that would only identify points having the same image under $f_{\left(i_{1}, \ldots, i_{d}\right)}$, while restricting ourselves to operations where we could control homeomorphism type and regularity. To this end, we collapse cells across families of curves which seem typical enough of fibers of maps of interest arising e.g. in combinatorial representation theory to be likely to be useful for other examples of interest as well.

While these collapses are topological in nature, we have gone to considerable effort to make the criteria one must check in order to use them as combinatorial as possible. This is done not only to help us with the proof of Conjecture 1.1, but also to facilitate possible future applications to other stratified spaces of interest in combinatorics and representation theory such as the double Bruhat decomposition for the totally nonnegative part of the Grassmannian or the totally nonnegative part of the flag variety, as discussed briefly in Sect. 1.2. Taken together, Theorem 1.3 and Theorem 4.21 provide a fairly combinatorial general approach to proving that images of sufficiently nice maps from polytopes are regular $\mathrm{CW}$ complexes homeomorphic to balls.

Another crucial ingredient in the proof of Conjecture 1.1 is the 0 -Hecke algebra associated to a Coxeter group $W$. The relations of the 0-Hecke algebra capture in a completely natural way which faces of a simplex (indexed by subwords of a reduced word) map to the same cell under $f_{\left(i_{1}, \ldots, i_{d}\right)}$, and in fact provide a dictionary from the topology of point identifications to the combinatorics of cell identifications through suppression of a parameter. Checking the requirements for our collapses thereby translates to an analysis of properties of reduced and nonreduced words in this 0-Hecke algebra.

While $f_{\left(i_{1}, \ldots, i_{d}\right)}$ itself is not a homeomorphism, we use the aforementioned collapses to construct a quotient space upon which the induced map $\overline{f_{\left(i_{1}, \ldots, i_{d}\right)}}$ will act homeomorphically, allowing us to understand its image based upon our understanding of this quotient space. These collapses eliminate exactly the faces of a simplex indexed by the subwords of a reduced word $\left(i_{1}, \ldots, i_{d}\right)$ that are themselves non-reduced. Theorem 1.3 gives a way then to prove that the induced map $\overline{f_{\left(i_{1}, \ldots, i_{d}\right)}}$ on the resulting quotient space is a homeomorphism. This is what guarantees that no further identification is necessary once 
we have performed all of the identifications which the non-reduced subwords necessitate.

The remainder of the introduction gives a more thorough overview of the main ideas going into the proof of the Fomin-Shapiro Conjecture, including the new tools leading up to it, then briefly discusses other possible future applications of our approach. Section 2 provides background and terminology in topology, topological combinatorics, Coxeter groups and their 0-Hecke algebras, and in total positivity theory, respectively; readers might find it useful to read one or more of these background sections even prior to reading the proof overview. Sections 3 and 4 establish the key topological tools, namely Theorem 1.3 (our regularity criterion for $\mathrm{CW}$ complexes) and Theorem 4.21 (our method for collapsing cells), respectively. Section 5 develops combinatorial properties of the 0 -Hecke algebra.

Then Sect. 6 pulls this all together in the (mainly combinatorial) proof of Conjecture 1.1, with the most difficult combinatorics appearing in Lemma 6.30. Theorem 6.32 assembles the various lemmas which together imply that the complex resulting from our series of collapses is indeed regular and homeomorphic to a ball. Finally, the Fomin-Shapiro Conjecture is proven in Theorem 6.34. Throughout the paper, we deliberately include a high level of detail, so as to help readers bridge between the combinatorics, topology, and representation theory.

\subsection{Proof overview}

Following Lusztig [20], we realize the stratified spaces $Y_{w}$ from Conjecture 1.1 as images of maps $f_{\left(i_{1}, \ldots, i_{d}\right)}$ from polytopes (which in our case are simplices) to spaces of matrices.

Let $\left(i_{1}, \ldots, i_{d}\right)$ be a reduced word for $w \in W$. Consider the surjective map $f_{\left(i_{1}, \ldots, i_{d}\right)}: \mathbb{R}_{>0}^{d} \rightarrow Y_{w}$ sending $\left(t_{1}, \ldots, t_{d}\right)$ to the product of matrices $x_{i_{1}}\left(t_{1}\right) \cdots x_{i_{d}}\left(t_{d}\right)$ where $x_{i}(t)=I_{n}+t E_{i, i+1}$ in type A, and more generally $x_{i}(t)=\exp \left(t e_{i}\right)$ for $e_{i}$ a Chevalley generator. Lusztig proved that $f_{\left(i_{1}, \ldots, i_{d}\right)}$ applied to $\mathbb{R}_{>0}^{d}$ is a homeomorphism. On the other hand, $f_{\left(i_{1}, \ldots, i_{d}\right)}$ is far from injective on $\mathbb{R}_{\geq 0}^{d}$, due to the relations (a) $x_{i}(u) x_{i}(v)=x_{i}(u+v)$ and (b) the type A braid relations

(1) $x_{i}(u) x_{j}(v)=x_{j}(v) x_{i}(u)$ for $|j-i|>1$

(2) $x_{i}(a) x_{i+1}(b) x_{i}(c)=x_{i+1}\left(\frac{b c}{a+c}\right) x_{i}(a+c) x_{i+1}\left(\frac{a b}{a+c}\right)$ for $a, b, c>0$

and similar relations $x_{i}\left(t_{1}\right) x_{j}\left(t_{2}\right) x_{i}\left(t_{3}\right) \cdots=x_{j}\left(t_{1}^{\prime}\right) x_{i}\left(t_{2}^{\prime}\right) x_{j}\left(t_{3}^{\prime}\right) \cdots$ of degree $m(i, j)$ in other types, where $m(i, j)$ is the order of $s_{i} s_{j}$ and $\left(t_{1}^{\prime}, \ldots, t_{m(i, j)}^{\prime}\right)$ is obtained from $\left(t_{1}, \ldots, t_{m(i, j)}\right)$ by a change of coordinates map as in [20]. We study the image of $f_{\left(i_{1}, \ldots, i_{d}\right)}$ restricted to the intersection of $\mathbb{R}_{\geq 0}^{d}$ with the hyperplane $\sum t_{i}=1$, denoting this domain by $\mathbb{R}_{\geq 0}^{d} \cap S_{1}^{d-1}$. This has the 
benefit of being compact while already reflecting the full structure of the image of $f_{\left(i_{1}, \ldots, i_{d}\right)}$ on domain $\mathbb{R}_{\geq 0}^{d}$. This domain is a simplex, with faces specified by which parameters $t_{i}$ are positive and which are 0 . Since $x_{i}(0)$ is the identity, it is natural to index the faces of the simplex by the subwords of $\left(i_{1}, \ldots, i_{d}\right)$. Lusztig's result for $\mathbb{R}_{>0}^{d}$ together with the above relations implies that $f_{\left(i_{1}, \ldots, i_{d}\right)}$ restricted to the interior of a face is injective if and only if the subword of $\left(i_{1}, \ldots, i_{d}\right)$ indexing that face is a reduced word.

The above relations will enable us to construct for any face of the simplex indexed by a nonreduced word a family of curves covering the face such that each curve lives in a single fiber of $f_{\left(i_{1}, \ldots, i_{d}\right)}$. These curves result from the relations $x_{i}(u) x_{i}(v)=x_{i}(u+v)$ either directly or after a suitable series of (long and short) braid moves. We will collapse each such non-reduced face across a family of such curves. While every nonreduced expression will admit a series of braid moves leading to such a "stutter" $x_{i}(u) x_{i}(v)$, a serious challenge to be overcome is that the requisite long braid moves give change of coordinate maps which a priori are not even well-defined on the closures of the cells to be collapsed, let alone homeomorphisms on them.

We get around this by doing certain other collapses earlier than a collapse requiring long braid moves. Specifically, we choose the collapsing order so that earlier identifications in the boundary of a cell requiring long braid moves will ensure that the change of coordinates map will in fact be a well-defined homeomorphism on each closed cell to be collapsed just prior to its collapse. The key conceptual lemma behind these change of coordinates maps being homeomorphisms is Lemma 6.20, while the technical details are handled in Lemma 6.24.

To see which faces of the simplex should be identified with each other in this manner, we suppress parameters, replacing $x_{i}(t)$ by $x_{i}$ for each $t$ that is positive, omitting the letters where $t$ is 0 . We thereby associate a so-called $x$-expression to each face. An examination of which $x$-expressions correspond to faces having the same image under $f_{\left(i_{1}, \ldots, i_{d}\right)}$ yields the relations (1) $x_{i}^{2}=x_{i}$ together with the braid relations (2) $x_{i} x_{j}=x_{j} x_{i}$ for $|j-i|>1$ and (3) $x_{i} x_{i+1} x_{i}=x_{i+1} x_{i} x_{i+1}$ in type A. Going beyond type A, we replace (2) and (3) by analogous long braid relations $x_{i} x_{j} x_{i} \cdots=x_{j} x_{i} x_{j} \cdots$ of degree $m(i, j)$ for each pair of Coxeter group generators $\left\{s_{i}, s_{j}\right\}$. In this manner, the (unsigned) 0 -Hecke algebra of the Coxeter group $W$ emerges. Two faces of the simplex will have the same image under $f_{\left(i_{1}, \ldots, i_{d}\right)}$ exactly when their $x$-expressions represent the same element of the 0 -Hecke algebra, or equivalently, in the language of [18] if they have the same Demazure product.

In Sect. 4, we introduce a general class of collapsing maps which may be performed sequentially on a polytope, preserving homeomorphism type and regularity on the resulting quotient cell complexes at each step. Each such map is defined by first covering a polytope face with a family of parallel line segments across which the face is collapsed, or more generally in subsequent 
steps covering a cell to be collapsed with a family of curves which we call parallel-like (see Definition 4.7), due to their being the image of a family of parallel line segments under a map $g$ with certain convenient properties (such as being a homeomorphism on the interior of the cell to be collapsed). The following simple example already captures much of the idea of our collapses.

Example 1.4 Let $\Delta_{2}$ be the convex hull of $(0,0),(1,0),(0,1 / 2)$ in $\mathbb{R}^{2}$, and let $\Delta_{1}$ be the convex hull of $(0,0)$ and $(1,0)$ in $\mathbb{R}^{2}$. We will construct a surjective, continuous function $h: \mathbb{R}^{2} \rightarrow \mathbb{R}^{2}$ that acts homeomorphically on $\mathbb{R}^{2}-\Delta_{2}$ sending it to $\mathbb{R}^{2}-\Delta_{1}$. The idea is to map parallel, vertical line segments covering $\Delta_{2}$ onto their endpoints in $\Delta_{1}$, then take a neighborhood $N$ of $\Delta_{2}$, specifically a collar for $\overline{\mathbb{R}^{2}-\Delta_{2}}$, and define $h$ in such a way that it stretches $N$ to cover $\Delta_{2}$ by mapping extensions of the parallel line segments surjectively onto the extended segments. For $0 \leq x \leq 1$ and $0 \leq y \leq-x / 2+1 / 2$, let $h(x, y)=(x, 0)$. For $0 \leq x \leq 1$ and $-x / 2+1 / 2 \leq y \leq 1$, let $h(x, y)=\left(x, \frac{y-1 / 2+x / 2}{1 / 2+x / 2}\right)$. For $-1 \leq x \leq 0$ and $0 \leq y \leq-x / 2+1 / 2$, let $h(x, y)=\left(x, y \frac{-x}{-x / 2+1 / 2}\right)$. For $-1 \leq x \leq 0$ and $-x / 2+1 / 2 \leq y \leq 1$, let $h(x, y)=(x,-1+2 y)$. Let $h$ act as the identity outside $R=\{(x, y):-1 \leq x \leq 1,0 \leq y \leq 1\}$.

Remark 1.5 See pp. 42-43 in Spanier [32] for a closely related, though fundamentally different, homeomorphism also given by explicit maps.

Each curve in a family of parallel-like curves will have one endpoint in a closed cell $G_{1}$ in the boundary of the cell $F$ to be collapsed, and the other endpoint in another closed boundary cell $G_{2}$. In Example 1.4, this is the segment from $(0,1 / 2)$ to $(1,0)$, and the segment from $(0,0)$ to $(1,0)$, respectively. The collapse given by this family of curves will map each curve to its endpoint in $G_{2}$, stretching a collar for the closed complement of $F$ within the boundary of a cell of dimension one higher than $F$ so as to homeomorphically cover $F$ by the part of this closed collar given by $G_{1} \times[0,1]$. Convexity of each face of the polytope whose image we are studying will enable this stretching to be accomplished by a continuous map. Corollary 4.24 generalizes our collapsing maps somewhat beyond polytopes to help accommodate requisite changes of coordinates, using that these collapsing maps may be transferred from one regular $\mathrm{CW}$ ball to a homeomorphic one, provided that both have the same cell structure on the closed cell to be collapsed, with the homeomorphism of regular $\mathrm{CW}$ complex restricting to a cell structure preserving one on this closed cell.

To relate this to our main application, notice e.g. that $\left\{\left(t_{1}, t_{2}, t_{3}\right) \in \mathbb{R}_{\geq 0}^{3} \mid t_{1}+\right.$ $t_{2}=k_{1,2}$ and $\left.t_{3}=k_{3}\right\}$ for the various choices of constants $k_{1,2}, k_{3}$ adding to 1 give parallel line segments covering the simplex $\left\{\left(t_{1}, t_{2}, t_{3}\right) \in \mathbb{R}_{\geq 0}^{3} \mid \sum t_{i}=1\right\}$ and comprising exactly the fibers of the map $\left(t_{1}, t_{2}, t_{3}\right) \mapsto x_{1}\left(t_{1}\right) x_{1}\left(t_{2}\right) x_{2}\left(t_{3}\right)$. 
All of our families of parallel-like curves will result either directly from stuttering pairs $x_{i}(u) x_{i}(v)$ of consecutive letters in a non-reduced expression yielding such parallel line segments, or as families of curves obtained from such parallel line segments by the composition of a series of earlier collapsing maps (that restrict to homeomorphisms on the interior of the cell now under consideration) with the change of coordinate homeomorphisms given by the long braid moves used to create a stuttering pair.

We require the following properties for parallel-like curves:

(1) Distinct initial points condition (DIP): the endpoints of the parallel-like curves in $G_{1}$ are distinct.

(2) Distinct endpoints condition (DE): for each nontrivial curve in the collection, its endpoints in $G_{1}$ and $G_{2}$ are distinct, by virtue of open cells in $G_{1}$ and $G_{2}$, respectively, not having already been identified with each other.

We also require a combinatorial property of the collapses themselves:

- Least upper bound condition (LUB): whenever two cells are identified by the collapse of a cell that is a least upper bound for the pair of them just prior to the collapse, then all cells that are least upper bounds for them just prior to the collapse also get collapsed in that same step.

The condition (DIP) is needed for the collapsing map across parallel-like curves to be well-defined, since the endpoint of each curve in $G_{1}$ (along with the rest of the curve) is mapped onto the other endpoint of the curve, which is in $G_{2}$. Condition (DE) will allow us to extend the parallel-like curves across a collar for the closed complement of the cell being collapsed within the boundary of a cell of dimension one higher, once we prove such a collar exists. The proof that regularity is preserved under our collapses relies heavily on (LUB).

Collapses meeting the more precisely formulated versions of these conditions given later will automatically meet a further condition we call the inductive manifold condition, namely that the closure of the complement of an $i$-cell within the boundary of an $(i+1)$-cell is a compact topological manifold with boundary, hence has a collar. This will allow us to extend the collapsing map for a low dimensional cell $F$ from a low-dimensional subcomplex where it is most naturally defined to our entire complex; this extension process in based upon the existence of requisite collars together with the fact that our particular collapsing maps admit approximations by homeomorphisms; in fact, we use that each comes with a path of homeomorphisms to the identity map, enabling each collapsing map to be deformed to the identity map across the layers of a collar.

Checking the above conditions for a family of curves covering a cell to be collapsed in our main application will rely on a combinatorial analysis of which cells have been identified with each other at the time of each collapse. In preparation for these combinatorial arguments, we develop in Sect. 5 
properties of the 0-Hecke algebra, based mainly on the following convenient notion: we say that a pair of letters $\left\{i_{r}, i_{s}\right\}$ for $r<s$ in a non-reduced word $\left(i_{1}, \ldots, i_{d}\right)$ is a deletion pair if $\left(i_{r}, \ldots, i_{s-1}\right)$ and $\left(i_{r+1}, \ldots, i_{s}\right)$ are both reduced while $\left(i_{r}, \ldots, i_{s}\right)$ is non-reduced. The 0 -Hecke algebra lacks a cancellation law, adding to the challenge of working with it. However, focusing on deletion pairs will enable some critical properties of Coxeter groups to be transferred to the 0-Hecke algebra, using the fact that reduced expressions in the 0 -Hecke algebra are exactly the reduced expressions in the associated Coxeter group.

Section 6 gives a particular series of cell collapses, performed sequentially on a simplex $\mathbb{R}_{>0}^{d} \cap S_{1}^{d-1}$, to produce a regular CW complex $\left(\mathbb{R}_{>0}^{d} \cap S_{1}^{d-1}\right) / \sim$ homeomorphic to a ball. The guiding principle behind our choice of ordering is that we want a collapsing order amenable to proof by induction on $d$. This will allow us to assume all our results for smaller $d$; this guarantees when we perform a long braid move on the expression associated to a face to be collapsed that all possible identifications based on subexpressions of the expression to be braided will have already been done. To this end, we collapse faces in an order consistent with linear order on the position of the right endpoint of the leftmost deletion pair in the (highest priority) $x$-expression representing that face. This implies at the time of the collapse of a face whose leftmost deletion pair is $\left\{i_{r}, i_{s}\right\}$ that all possible point identifications based on letters strictly to the left of position $s$ will have already been done, so that we may apply braid moves to the segment from positions $r$ through $s-1$ so as to create a stutter with the letter at position $s$.

In this manner, we collapse away all faces of the simplex indexed by nonreduced words based on point and cell identifications which are clearly necessary. However, this still leaves the challenge of proving that these identifications are enough, that there are no remaining instances of two points mapping to the same place under the induced quotient space map $\bar{f}_{\left(i_{1}, \ldots, i_{d}\right)}$. This is where we turn to Theorem 1.3, to prove that $\bar{f}_{\left(i_{1}, \ldots, i_{d}\right)}$ is indeed a homeomorphism from $\left(\mathbb{R}_{\geq 0}^{d} \cap S_{1}^{d-1}\right) / \sim$ to $Y_{w}$.

Corollary 3.9 sets up the framework in which Theorem 1.3 will be used, both in this paper and most likely in other applications as well. It focuses on images of maps from regular $\mathrm{CW}$ complexes having a maximal cell, with the further requirement that the map be a homeomorphism on this open big cell. It singles out conditions 3 and 4 of Theorem 1.3 to be checked for the particular application, with conditions 1,2 and 5 then following automatically from the general set-up.

In the setting of the Fomin-Shapiro Conjecture, condition 3 is immediate from the result of Björner and Wachs that Bruhat order is thin and shellable [6]. The idea we will use in Lemma 6.33 to verify condition 4 is as follows. Given a reduced expression $s_{i_{1}} \cdots s_{i_{d}}$, the Coxeter group element 
obtained by deleting a single letter $s_{i_{u}}$ cannot be the same as the Coxeter group element obtained by deleting a letter $s_{i_{v}}$ for $u \neq v$. This combines with Lusztig's result that $f_{\left(i_{1}, \ldots, i_{d}\right)}$ restricts to a homeomorphism on the interior of a cell indexed by reduced word $\left(i_{1}, \ldots, i_{d}\right)$ to allow us to verify codimension one injectivity. The point is that approaching the boundary of a cell by letting a single parameter $t_{i}$ go to 0 as opposed to approaching the boundary by letting a different, individual parameter $t_{j}$ go to 0 must give points in distinct cells, hence distinct points.

We will use the map $\bar{f}_{\left(i_{1}, \ldots, i_{d}\right)}:\left(\mathbb{R}_{\geq 0}^{d} \cap S_{1}^{d-1}\right) / \sim \rightarrow Y_{w}$ induced from $f_{\left(i_{1}, \ldots, i_{d}\right)}$ along with its restriction to various closed cells as the characteristic maps with respect to which we will prove that $Y_{w}$ is a regular CW complex. Our results will imply that $\sim$ identifies exactly those points having the same image under Lusztig's map $f_{\left(i_{1}, \ldots, i_{d}\right)}:\left(t_{1}, \ldots, t_{d}\right) \rightarrow x_{i_{1}}\left(t_{1}\right) \cdots x_{i_{d}}\left(t_{d}\right)$ given by any reduced word $\left(i_{1}, \ldots, i_{d}\right)$ for $w \in W$. We will prove in Theorem 6.34 that $\bar{f}_{\left(i_{1}, \ldots, i_{d}\right)}$ is a homeomorphism from $\left(\mathbb{R}_{\geq 0}^{d} \cap S_{1}^{d-1}\right) / \sim$ to $Y_{s_{i_{1}} \cdots s_{i_{d}}}$ with $\bar{f}_{\left(i_{1}, \ldots, i_{d}\right)}$ sending the open cells of $\left(\mathbb{R}_{\geq 0}^{d} \cap S_{1}^{d-1}\right) / \sim$ to the cells $Y_{u}^{o}$ with $u \leq w=s_{i_{1}} \cdots s_{i_{d}}$, completing the proof of Conjecture 1.1.

\subsection{Potential further applications}

Lusztig and Rietsch have studied a combinatorial decomposition for the totally nonnegative part of a flag variety, namely the decomposition into double Bruhat cells (cf. [20] and [28]). Lusztig proved contractibility of the entire space in [20] while Rietsch and Williams proved contractibility of each cell closure in [30]. Williams conjectured in [35] that this is a regular CW complex homeomorphic to a ball. It seems quite plausible that Theorem 1.3 together with tools from Sect. 4 could also be used to prove that conjecture, though we believe that significant further new ideas would also be needed.

Rietsch determined the closure poset of this decomposition in [28]. Williams proved in [35] that this poset is shellable and thin, implying it meets Condition 3 of Theorem 1.3. Postnikov, Speyer and Williams proved in [26] in the case of the Grassmannian that its double Bruhat decomposition is a CW decomposition; Rietsch and Williams subsequently generalized this to all flag varieties in [29]. In each case, it remains open whether these CW complexes are regular and whether the spaces themselves are homeomorphic to balls.

Remark 1.6 Williams' conjecture is related to Conjecture 1.1 in that the stratified spaces we prove to be regular CW complexes arise as links of cells in the double Bruhat stratification of the flag variety. However, Williams' conjecture does not imply Conjecture 1.1 since links of cells in regular $\mathrm{CW}$ complexes are not always themselves regular. Consider e.g. the double suspension of a Poincare homology 3-sphere with a big cell glued in (personal communication, Anders Björner). 
In the case of an arbitrary flag variety, the preimage polytope that has been constructed in [29] is quite abstract, guaranteed to exist by properties of canonical bases. In the special case of the Grassmannian, much more explicit combinatorics is known about Postnikov's polytope of plabic graphs as well as the map from this polytope to the totally nonnegative part of the Grassmannian (see [25]).

\section{Background and Terminology}

Now we collect together basic terminology and facts from topology, combinatorics, the theory of Coxeter groups, and total positivity theory that will be essential to this paper. See e.g. [4, 5, 7, 15, 16, 20, 24, 31, 32], or [33] for further details.

\subsection{Background in topology}

Definition 2.1 A CW complex is a space $X$ and a collection of disjoint open cells $e_{\alpha}$ whose union is $X$ such that:

(1) $X$ is Hausdorff.

(2) For each open $m$-cell $e_{\alpha}$ of the collection, there exists a continuous map $f_{\alpha}: B^{m} \rightarrow X$ that maps the interior of $B^{m}$ homeomorphically onto $e_{\alpha}$ and carries the boundary of $B^{m}$ into a finite union of open cells, each of dimension less than $m$.

(3) A set $A$ is closed in $X$ if $A \cap \bar{e}_{\alpha}$ is closed in $\bar{e}_{\alpha}$ for each $\alpha$.

An open $m$-cell is any topological space which is homeomorphic to the interior of an $m$-ball $B^{m}$, with an open 0-cell being a point. The restriction of a characteristic map $f_{\alpha}$ to the boundary of $B^{m}$ is an attaching map. Denote the closure of a cell $\alpha$ by $\bar{\alpha}$. A finite $C W$ complex is a CW complex with finitely many open cells.

Definition 2.2 A CW complex is regular with respect to $\left\{f_{\alpha}\right\}$ if additionally each $f_{\alpha}$ restricts to a homeomorphism from the boundary of $B^{m}$ onto a finite union of lower dimensional open cells.

The following (which appears as Theorem 38.2 in [24]) will enable us to build $\mathrm{CW}$ complexes by induction on dimension.

Theorem 2.3 Let $Y$ be a CW complex of dimension at most $p-1$, let $\sum B_{\alpha}$ be a topological sum of closed p-balls, and let $g: \sum B d\left(B_{\alpha}\right) \rightarrow Y$ be a continuous map. Then the adjunction space $X$ formed from $Y$ and $\sum B_{\alpha}$ by means of $g$ is a CW complex, and $Y$ is its $(p-1)$-skeleton. 
Definition 2.4 Let $g: X \rightarrow Y$ be a continuous, surjective function. Then the quotient topology on $Y$ is the topology whose open sets are the sets whose inverse images are open in $X$. Say that $g$ is an identification map if the topology on $Y$ is the quotient topology induced by $g$.

The requirement needed for a continuous, surjective $g: X \rightarrow Y$ to be an identification map is automatic if $X, Y$ are compact and Hausdorff, which will always hold for our upcoming collapsing maps.

Remark 2.5 Given an identification map $g: X \rightarrow Y$ and a function $f$ : $X \rightarrow Z$ such that $g(x)=g(y)$ implies $f(x)=f(y)$, then Proposition 13.5 of [Br] implies that $f$ is continuous iff the induced function $\bar{f}: Y \rightarrow Z$ satisfying $f=\bar{f} \circ g$ is continuous.

Remark 2.5 will allow us to use continuity of $f_{\left(i_{1}, \ldots, i_{d}\right)}$ to deduce continuity of the induced map $\overline{f_{\left(i_{1}, \ldots, i_{d}\right)}}$ on the quotient space after a series of collapses, each of which is given by an identification map.

Definition 2.6 A topological $n$-manifold is a Hausdorff space $M$ having a countable basis of open sets, with the property that every point of $M$ has a neighborhood homeomorphic to an open subset of $\mathbb{H}^{n}$, where $\mathbb{H}^{n}$ is the halfspace of points $\left(x_{1}, \ldots, x_{n}\right)$ in $\mathbb{R}^{n}$ with $x_{n} \geq 0$. The boundary of $M$, denoted $\partial M$, is the set of points $x \in M$ for which there exists a homeomorphism of some neighborhood of $x$ to an open set in $\mathbb{H}^{n}$ taking $x$ into $\left\{\left(x_{1}, \ldots, x_{n}\right) \mid x_{n}=\right.$ $0\}=\partial \mathbb{H}^{n}$.

Next we review the notion of a collaring, since this will be critical to our general construction of cell collapses in Theorem 4.21.

Definition 2.7 Given a topological manifold $M$ with boundary, a collar or collaring for $M$ is a closed neighborhood $N$ of $\partial M$ contained in $M$ that is homeomorphic to $\partial M \times[0,1]$ with $\partial M$ mapping to $\partial M \times\{0\}$.

A proof of the following appears in [8] and in Appendix II of [34].

Theorem 2.8 If $M$ is a compact, topological manifold with boundary $\partial M$, then $M$ has a collar.

We will extend collapsing maps from low-dimensional cell boundaries in which they are most naturally defined to higher dimensional cells by proving existence of requisite collars and showing that our collapsing maps can be approximated by homeomorphisms, in fact constructing a continuous path of homeomorphisms from our collapsing map (which itself is not a homeomorphism) to the identity map. 
Finally, we briefly recall from [14] the notion of link for Whitney stratified spaces, and we refer the reader to [14] for further details. Let $Z$ be a Whitney stratified subset of a smooth manifold $M$, let $N^{\prime}$ be a smooth submanifold which contains a given point $p$ of $Z$ and which is transverse to each stratum of $Z$ containing $p$. Let $B_{\delta}(p)$ be the closed ball of radius $\delta$ centered at $p$. Then the link, denoted $L(p)$, of a stratum $S$ at the point $p$ is the set $L(p)=$ $N^{\prime} \cap Z \cap \partial D_{\delta}(p)$.

\subsection{Background in topological combinatorics}

Definition 2.9 The closure poset of a finite CW complex is the partially ordered set (or poset) of open cells with $\sigma \leq \tau$ iff $\sigma \subseteq \bar{\tau}$. By convention, we adjoin a unique minimal element $\hat{0}$ which is covered by all the 0 -cells, which may be regarded as representing the empty set.

Let $\partial \tau$ denote $\bar{\tau} \backslash \tau$, i.e. the boundary of $\bar{\tau}$.

Definition 2.10 The order complex of a finite partially set is the simplicial complex whose $i$-dimensional faces are the chains $u_{0}<\cdots<u_{i}$ of $i+1$ comparable poset elements.

A poset is graded if for each $u \leq v$, all saturated chains $u=u_{0} \prec u_{1} \prec$ $\cdots \prec u_{k}=v$ involve the same number $k$ of covering relations $u_{i} \prec u_{i+1}$ (i.e. $u_{i}<u_{i+1}$ such that $u_{i} \leq v \leq u_{i+1}$ implies $v=u_{i}$ or $v=u_{i+1}$ ). In this case, we say that the poset interval $[u, v]$ has rank $k$. Recall that a finite, graded poset with unique minimal and maximal elements is Eulerian if each interval $[u, v]$ has equal numbers of elements at even and odd ranks. This is equivalent to its Möbius function satisfying $\mu(u, v)=(-1)^{r k(v)-r k(u)}$ for each pair $u<v$, or in other words the order complex of each open interval $(u, v)$ having the same Euler characteristic as that of a sphere $S^{r k(v)-r k(u)-2}$. A finite, graded poset is thin if each rank two closed interval $[u, v]$ has exactly four elements, in other words if each such interval is Eulerian.

Remark 2.11 The order complex of the closure poset of a finite regular CW complex $K$ (with $\hat{0}$ removed) is the first barycentric subdivision of $K$, hence is homeomorphic to $K$. In particular, this implies that the order complex for any open interval $(\hat{0}, v)$ in the closure poset of $K$ will be homeomorphic to a sphere $S^{r k(v)-2}$.

In [4], Björner characterized which finite, graded posets are closure posets of regular $\mathrm{CW}$ complexes, calling such posets $C W$ posets:

Theorem 2.12 (Björner) A finite, graded poset with unique minimal element $\hat{0}$ is the closure poset of a regular CW complex if and only if (1) it has at least 
one additional interval, and (2) each open interval $(\hat{0}, u)$ has order complex homeomorphic to a sphere $S^{r k(u)-2}$.

Results of Danaraj and Klee in [9] give a convenient way to verify (2) for a finite, graded poset $P$, namely by proving $P$ is thin and shellable.

Remark 2.13 Two finite CW complexes may have the same closure poset in spite of having very different topological structure, so proving that the closure poset of a stratified space is a CW poset gives evidences that the stratified space is a regular CW complex, but is not enough to determine topological structure of the stratified space itself.

Definition 2.14 A convex polytope is the convex hull of a finite collection of points in $\mathbb{R}^{n}$, or equivalently it is an intersection of closed half spaces that is bounded.

For simplicial complexes and polytopes, the closure poset is often called the face poset. Let $[\sigma, \tau]$ denote the subposet consisting of elements $z$ such that $\sigma \leq z \leq \tau$, called the closed interval from $\sigma$ to $\tau$. Likewise, the open interval from $\sigma$ to $\tau$, denoted $(\sigma, \tau)$, is the subposet of elements $z$ with $\sigma<$ $z<\tau$. A cell $\sigma$ covers a cell $\rho$, denoted $\rho \prec \sigma$, if $\rho<\sigma$ and each $z$ with $\rho \leq z \leq \sigma$ must satisfy $z=\rho$ or $z=\sigma$.

For a regular cell complex in which the link of any cell is also regular, $\Delta(u, v)$ is homeomorphic to the link of $u$ within the boundary of $v$, hence is homeomorphic to $S^{\operatorname{dim}(v)-\operatorname{dim}(u)-2}$.

Remark 2.15 If each closed interval $[u, v]$ of a finite poset $P$ is Eulerian and shellable, then each open interval has order complex homeomorphic to a sphere $S^{r k(v)-r k(u)-2}$, implying condition 3 of Theorem 1.3.

The stratified spaces we consider in our main application have closure posets that are the intervals of Bruhat order, which were proven to be thin and shellable by Björner and Wachs in [6].

\subsection{Background on Coxeter groups and their associated 0-Hecke algebras}

Let $s_{i}$ denote the adjacent transposition $(i, i+1)$ swapping the letters $i$ and $i+1$ in type A, and more generally denote a member of a minimal set of generators called the simple reflections of a Coxeter $W$ group by $\left\{s_{i} \mid i \in I\right\}$. Its relations are all of the form $\left(s_{i} s_{j}\right)^{m(i, j)}=e$ with $m(i, i)=2$ for all $i$ and $m(i, j) \geq 2$ otherwise. Finite Weyl groups are all Coxeter groups.

An expression for a Coxeter group element $w$ is a way of writing it as a product of simple reflections $s_{i_{1}} \cdots s_{i_{r}}$. An expression is reduced when 
it minimizes $r$ among all expressions for $w$, in which case $r$ is called the length of $w$. An expression $s_{i_{1}} \cdots s_{i_{d}}$ may be represented more compactly by its word, namely by $\left(i_{1}, \ldots, i_{d}\right)$. Breaking now from standard terminology, we also speak of the wordlength of a (not necessarily reduced) expression $s_{i_{1}} \cdots s_{i_{r}}$, by which we again mean $r$. Given simple reflections $s_{i}, s_{j}$, define $m(i, j)$ to be the least positive integer such that $\left(s_{i} s_{j}\right)^{m(i, j)}=1$.

The following basic lemma will be key to our proof that the complexes $Y_{w}$ satisfy Condition 4 in our $\mathrm{CW}$ complex regularity criterion:

Lemma 2.16 Given a reduced word $s_{i_{1}} s_{i_{2}} \cdots s_{i_{r}}$ for a Coxeter group element $w$, any two distinct subwords of length $r-1$ which are both themselves reduced must give rise to distinct Coxeter group elements.

We include a short proof of this vital fact for completeness sake.

Proof Suppose deleting $s_{i_{j}}$ yields the same Coxeter group element which we get by deleting $s_{i_{k}}$ for some pair $1 \leq j<k \leq r$. This implies $s_{i_{j}} s_{i_{j+1}} \cdots s_{i_{k-1}}=$ $s_{i_{j+1}} \cdots s_{i_{k-1}} s_{i_{k}}$. Multiplying on the right by $s_{i_{k}}$ yields

$$
s_{i_{j}} s_{i_{j+1}} \cdots s_{i_{k-1}} s_{i_{k}}=s_{i_{j+1}} \cdots s_{i_{k-1}}\left(s_{i_{k}}\right)^{2}=s_{i_{j+1}} \cdots s_{i_{k-1}},
$$

contradicting the fact that the original expression was reduced.

The expression $s_{1} s_{2} s_{1}$ in the symmetric group demonstrates that the statement of the above lemma no longer holds if we replace $r-1$ by $r-i$ for $i>1$. Thus, it really seems to be quite essential to our proof of the FominShapiro Conjecture that Theorem 1.3 enables us to focus on codimension one cell incidences.

Lemma 2.17 (Exchange Condition, [16]) Let $w=s_{1} \cdots s_{r}$ (not necessarily reduced) where each $s_{i}$ is a simple reflection. If $l(w s)<l(w)$ for some simple reflection $s=s_{\alpha}$, then there exists index $i$ for which $w s=s_{1} \cdots \hat{s}_{i} \cdots s_{r}$. In particular, $w$ has a reduced expression ending in s if and only if $l(w s)<l(w)$.

Given a (not necessarily reduced) expression $s_{i_{1}} \cdots s_{i_{d}}$ for a Coxeter group element $w$, define a braid-move to be the replacement of $s_{i} s_{j} s_{i} \ldots$ by $s_{j} s_{i} s_{j} \cdots$ yielding a new expression for $w$ by virtue of a braid relation $\left(s_{i} s_{j}\right)^{m(i, j)}=1$ with $i \neq j$. Define a nil-move to be the replacement of a substring $s_{i} s_{i}$ appearing in consecutive positions by 1 . We call braid moves with $m(i, j)=2$ commutation moves and those with $m(i, j)>2$ long braid moves.

Theorem 2.18 ([5], Theorem 3.3.1) Let $(W, S)$ be a Coxeter system consisting of Coxeter group $W$ and minimal generating set of simple reflections $S$. Consider $w \in W$. 
(1) Any expression $s_{i_{1}} s_{i_{2}} \cdots s_{i_{d}}$ for $w$ can be transformed into a reduced expression for $w$ by a sequence of nil-moves and braid-moves.

(2) Every two reduced expressions for $w$ can be connected via a sequence of braid-moves.

The Bruhat order is the partial order on the elements of a Coxeter group $W$ having $u \leq v$ iff there are reduced expressions $r(u), r(v)$ for $u, v$ with $r(u)$ a subexpression of $r(v)$. Bruhat order is also the closure order on the cells $B_{w}=B^{-} w B^{-}$of the Bruhat stratification of the reductive algebraic group having $W$ as its Weyl group.

Associated to any Coxeter system $(W, S)$ is a 0-Hecke algebra, with generators $\left\{x_{i} \mid i \in S\right\}$ and the following relations: for each braid relation $s_{i} s_{j} \cdots=s_{j} s_{i} \cdots$ in $W$, there is an analogous relation $x_{i} x_{j} \cdots=x_{j} x_{i} \cdots$, again of degree $m(i, j)$; there are also relations $x_{i}^{2}=-x_{i}$ for each $i \in S$. In our set-up, we will need relations $x_{i}^{2}=x_{i}$, but this sign change is inconsequential in our setting, so refer to the algebra with relations $x_{i}^{2}=x_{i}$ as the (unsigned) 0 -Hecke algebra of $W$. This variation on the usual 0-Hecke algebra has previously arisen in work on Schubert polynomials (see e.g. [12] or [23]). We refer to $x_{i}^{2} \rightarrow x_{i}$ as a modified nil-move. It still makes sense to speak of reduced and non-reduced expressions, and many properties (including Lemma 2.16 and Theorem 2.18) carry over to the 0 -Hecke algebra by virtue of having the same braid moves; there are important differences though too, largely resulting from the lack of inverses and a cancellation law.

\subsection{Background in total positivity theory}

Recall that a real matrix is totally nonnegative (resp. totally positive) if each minor is nonnegative (resp. positive). The totally nonnegative part of $S L_{n}(\mathbb{R})$ consists of the matrices in $S L_{n}(\mathbb{R})$ whose minors are all nonnegative. Motivated by connections to canonical bases, Lusztig generalized this dramatically in [20] as follows. The totally nonnegative part of a reductive algebraic group $G$ defined and split over $\mathbb{R}$ is the semigroup generated by the sets $\left\{x_{i}(t) \mid t \in\right.$ $\left.\mathbb{R}_{>0}, i \in I\right\},\left\{y_{i}(t) \mid t \in \mathbb{R}_{>0}, i \in I\right\}$, and $\left\{t \in T \mid \chi(t)>0\right.$ for all $\left.\chi \in X^{*}(T)\right\}$, for $I$ indexing the simple roots. In type A, we have $x_{i}(t)=I_{n}+t E_{i, i+1}$, namely the identity matrix modified to have the value $t$ in position $(i, i+1)$, and likewise, $y_{i}(t)=I_{n}+t E_{i+1, i}$. More generally, $x_{i}(t)=\exp \left(t e_{i}\right)$ and $y_{i}(t)=\exp \left(t f_{i}\right)$ for $\left\{e_{i}, f_{i} \mid i \in I\right\}$ the Chevallay generators. In other words, if we let $\phi_{i}$ be the homomorphism of $S L_{2}$ into $G$ associated to the $i$-th simple root, then

$$
x_{i}(t)=\phi_{i}\left(\begin{array}{ll}
1 & t \\
0 & 1
\end{array}\right) \quad \text { and } \quad y_{i}(t)=\phi_{i}\left(\begin{array}{ll}
1 & 0 \\
t & 1
\end{array}\right)
$$


Let $B^{+}, B^{-}$be opposite Borel subgroups with $N^{+}$(or simply $N$ ) and $N^{-}$ denoting their unipotent radicals. In type A, we may choose $B^{+}, B^{-}$to consist of the upper and lower triangular matrices in $G L(n)$, respectively. In this case, $N^{+}, N^{-}$are the matrices in $B^{+}, B^{-}$with diagonal entries all equalling one. The totally nonnegative part of $N^{+}$, denoted $Y$, is the submonoid generated by $\left\{x_{i}\left(t_{i}\right) \mid i \in I, t_{i} \in \mathbb{R}_{>0}\right\}$. Let $W$ be the Weyl group of $G$. One obtains a combinatorial decomposition of $Y$ by taking the usual Bruhat decomposition of $G$ and intersecting each open Bruhat cell $B_{w}=B^{-} w B^{-}$for $w \in W$ with $Y$ to obtain an open cell $Y_{w}^{o}:=Y \cap B_{w}$ in $Y$. We follow [20] in using the standard topology on $\mathbb{R}$ throughout this paper.

Theorem 2.19 (Lusztig) For $\left(i_{1}, \ldots, i_{d}\right)$ any reduced word for $w$ that the map $f_{\left(i_{1}, \ldots, i_{d}\right)}$ sending $\left(t_{1}, \ldots, t_{d}\right)$ to $x_{i_{1}}\left(t_{1}\right) \cdots x_{i_{d}}\left(t_{d}\right)$ is a homeomorphism from $\mathbb{R}_{>0}^{d}$ to $Y_{w}^{o}$ (see Proposition 2.7 in [20]).

The closure of $Y_{w}^{o}$, denoted $Y_{w}$, is the image of this same map applied to $\mathbb{R}_{\geq 0}^{d}$. Since $x_{i}(0)$ is the identity matrix, the cells in the closure of $Y_{w}^{o}$ are obtained by choosing subwords of $\left(i_{1}, \ldots, i_{d}\right)$, so $Y_{w}=\bigcup_{u \leq w} Y_{u}^{o}$ for $u \leq w$ in Bruhat order on $W$. Fomin and Shapiro suggested for each $u<w$ in Bruhat order that the link of the open cell $Y_{u}^{o}$ within $Y_{w}$ should serve as a good geometric model for the Bruhat interval $(u, w]$, namely as a naturally arising regular CW complex with $(u, w]$ as its closure poset. They define $l k(u, w)$ as we describe next.

Fomin and Shapiro introduced the following projection map $\pi_{u}: Y_{\geq u} \rightarrow$ $Y_{u}^{o}$. Letting $N(u)=u^{-1} B u \cap N$ and $N^{u}=B^{-} u B^{-} \cap N$, Fomin and Shapiro proved that each $x \in Y_{\geq u}$ has a unique expression as $x=x_{u} x^{u}$ with $x_{u} \in N^{u}$ and $x^{u} \in N(u)$. In light of results in [FS], $\pi_{u}(x)$ may be defined as equalling $x_{u} \in N^{u}$. They defined $l k(u, w)$ as $\left(\pi_{u}^{-1}\left(x_{u}\right)\right) \cap Y_{[u, w]} \cap S_{\epsilon}\left(x_{u}\right)$ for $x_{u}$ an arbitrary point in $Y_{u}^{o}$ and $S_{\epsilon}\left(x_{u}\right)$ a sufficiently small sphere about $x_{u}$ (cf. p. 11 in [11]). Thus, points of $l k(u, w)$ belong to cells $Y_{u^{\prime}}$ for $u<u^{\prime} \leq w$, and closure relations are inherited from $Y_{w}$. They proved that each of the proposed open cells in $l k(u, w)$ is indeed homeomorphic to $\mathbb{R}^{n}$ for some $n$.

Recall from [13, 20], the relations (1) $x_{i}\left(t_{1}\right) x_{j}\left(t_{2}\right)=x_{j}\left(t_{2}\right) x_{i}\left(t_{1}\right)$ for any $s_{i}, s_{j}$ which commute, and (2) $x_{i}\left(t_{1}\right) x_{j}\left(t_{2}\right) x_{i}\left(t_{3}\right)=x_{j}\left(\frac{t_{2} t_{3}}{t_{1}+t_{3}}\right) x_{i}\left(t_{1}+\right.$ $\left.t_{3}\right) x_{j}\left(\frac{t_{1} t_{2}}{t_{1}+t_{3}}\right)$ for any $s_{i}, s_{j}$ with $\left(s_{i} s_{j}\right)^{3}=e$ and any $t_{1}+t_{3} \neq 0$. These are not difficult to verify directly. In [20], it is proven that there are more general relations of a similar nature for each braid relation $\left(s_{i} s_{j}\right)^{m(i, j)}=e$ in $W$, i.e., relations $x_{i}\left(t_{1}\right) x_{j}\left(t_{2}\right) \cdots=x_{j}\left(t_{1}^{\prime}\right) x_{i}\left(t_{2}^{\prime}\right) \ldots$ of degree $m(i, j)$ for $t_{1}^{\prime}, \ldots, t_{m(i, j)}^{\prime}$ rational functions of $t_{1}, \ldots, t_{s}$ each mapping $\mathbb{R}_{>0}^{d}$ to $\mathbb{R}_{>0}$.

Lemma 2.20 The new parameters after applying a braid relation will have the same sum as the old ones; moreover, this preservation of sum refines to the subset of parameters for any fixed $x_{i}$. 
Proof This follows from the description of $x_{i}(t)$ as $\exp \left(t e_{i}\right)$, simply by comparing the linear terms in the expressions $x_{i}\left(t_{1}\right) x_{j}\left(t_{2}\right) \cdots=x_{j}\left(t_{1}^{\prime}\right) x_{i}\left(t_{2}^{\prime}\right) \ldots$ appearing in a braid relation.

Thus, our description of $\mathbb{R}_{>0}^{d} \cap S_{1}^{d-1}$ may be used even after a change of coordinates (as in Lemma 6.24) resulting from a braid relation.

\section{A new regularity criterion for $\mathrm{CW}$ complexes}

Before proving Theorem 1.3, the new regularity criterion, we first give a few examples demonstrating the need for its various hypotheses. It seems likely that this new criterion will mainly apply to images of regular CW complexes.

Example 3.1 The CW complex consisting of a 2-cell with its entire boundary attached to a 0 -cell violates condition 2 of Theorem 1.3. Condition 2 is designed also to preclude examples such as a $\mathrm{CW}$ complex whose 1-skeleton is the simplicial complex comprised of the faces $\left\{v_{1}, v_{2}, v_{3}, e_{1,2}, e_{1,3}, e_{2,3}\right\}$, also having a two cell with a nontrivial closed interval of its boundary mapped to $v_{2}$ and the remainder of its boundary mapped homeomorphically to the rest of the 1-skeleton.

Remark 3.2 In the latter example above, one may choose a different characteristic map which is a homeomorphism. Whether this can always be done for finite CW complexes graded by cell dimension and satisfying conditions 1 , 3,4 , and 5 seems subtle at best, in light of examples such as the Alexander horned sphere: a ball which cannot be contracted to a point without changing the topology of its complement, since that is not simply connected.

The next two examples give non-regular $\mathrm{CW}$ complex satisfying conditions $1,2,4$, and 5 of Theorem 1.3, but violating condition 3 . The first example violates thinness, while the second one violates the requirement that open intervals of rank at least 3 be connected.

Example 3.3 Let $K$ be a 2-dimensional $\mathrm{CW}$ complex whose 1-skeleton is the simplicial complex with maximal faces $\left\{e_{1,2}, e_{2,3}, e_{1,3}, e_{3,4}, e_{4,5}, e_{3,5}\right\}$ and which has a unique 2-cell $\sigma$. The boundary of $\sigma$ is mapped by $f_{\sigma}$ to the 1 cycle $\left(e_{3,1}, e_{1,2}, e_{2,3}, e_{3,4}, e_{4,5}, e_{5,3}\right)$. The attaching map $f_{\sigma}$ sends to different points of the boundary of $\sigma$ to $v_{3}$.

Example 3.4 For a non-regular CW complex satisfying conditions 1, 2, 4, and 5 of Theorem 1.3 as well as thinness of the closure poset, but violating the connectedness requirement for open intervals of rank at least 3, take a 3-dimensional cube and glue together a pair of antipodal vertices. 
One might ask if condition 3 could be replaced by the requirement that the closure poset be Eulerian, i.e. whether this could replace the connectedness part of the requirement. Closure posets do have the feature that open intervals $(\hat{0}, u)$ with $r k(u)>2$ are connected, by virtue of the fact that the image of a continuous map from a sphere $S^{d}$ with $d>0$ is connected. However, there are Eulerian closure posets of $\mathrm{CW}$ complexes having disconnected intervals $(u, v)$ with $r k(v)-r k(u)>2$ (personal communication, Hugh Thomas). Still, it seems plausible that condition 3 in Theorem 1.3 might be replaceable by the Eulerian property for the closure poset, i.e. that this together with conditions $1,2,4$, and 5 could imply regularity.

Next is a non-regular $\mathrm{CW}$ decomposition of $\mathbb{R} P_{2}$ satisfying conditions 1 , 2,3 , and 5 of Theorem 1.3, but failing condition 4 .

Example 3.5 Let $K$ be the CW complex having as its 1-skeleton the simplicial complex with maximal faces $e_{1,2}, e_{2,3}, e_{1,3}$. Additionally, $K$ has a single 2-cell whose boundary is mapped to the 1-cycle which goes twice around the 1 -cycle $\left(v_{1}, v_{2}, v_{3}\right)$. Notice that this $\mathrm{CW}$ decomposition of $\mathbb{R} P_{2}$ has the same closure poset as a 2-simplex, but the attaching map for the 2-cell is a 2 to 1 map onto the lower dimensional cells.

Finally, we give an example (due to David Speyer) of a CW complex with characteristic maps meeting conditions 1,2, 3 and 4, but failing condition 5, though this CW complex is regular with respect to a different choice of characteristic maps. David Speyer also helped with the formulation of condition 5.

Example 3.6 Let the 2-skeleton be the boundary of a pyramid. Now attach a 3-cell which is a triangular prism by sending an entire edge of one of the rectangular faces to the unique vertex of degree 4 in the pyramid, otherwise mapping the boundary of the prism homeomorphically to the boundary of the pyramid.

Proposition 3.7 Conditions 1 and 2 of Theorem 1.3 imply that the closure poset is graded by cell dimension.

Proof Consider any $e_{\rho} \subseteq \overline{e_{\sigma}}$ with $\operatorname{dim}\left(e_{\sigma}\right)-\operatorname{dim}\left(e_{\rho}\right)>1$. Choose a point $p$ in $e_{\rho}$ expressible as $f_{\sigma}(x)$ for some $x \in S^{\operatorname{dim}\left(e_{\sigma}\right)-1}$. If we take an infinite series of smaller and smaller open sets about $x$, by Condition 2 each must include a point sent by $f_{\sigma}$ to an open cell of higher dimension than $e_{\rho}$; finiteness of the CW complex then implies some such open cell $e_{\tau}$ is mapped into infinitely often, implying $p \in \overline{e_{\tau}}$. Thus, $e_{\rho}<e_{\sigma}$ for $\operatorname{dim}\left(e_{\sigma}\right)-\operatorname{dim}\left(e_{\rho}\right)>1$ implies there exists $e_{\tau}$ with $e_{\rho}<e_{\tau}<e_{\sigma}$. 
This motivates us to say that a finite $\mathrm{CW}$ complex is dimension-graded whenever it meets conditions 1 and 2 of Theorem 1.3. Now to the proof of Theorem 1.3.

Proof Conditions 1,2, and 4 are each necessary tautologically. The necessity of 3 follows easily from the fact that a regular $\mathrm{CW}$ complex is homeomorphic to the order complex of its closure poset. To see that 5 is also necessary, note that if $K$ is regular with respect to the characteristic maps $\left\{f_{\alpha}\right\}$, then $e_{\sigma} \subseteq \overline{e_{\tau}}$ implies that $f_{\sigma}$ factors as $\left.f_{\tau} \circ f_{\tau}^{-1}\right|_{\sigma} \circ f_{\sigma}$ where $\left.f_{\tau}^{-1}\right|_{\sigma} \circ f_{\sigma}$ is the desired embedding.

Now to the sufficiency of these five conditions. We must prove that each attaching map $f_{\sigma}$ is a homeomorphism from $\partial\left(B^{\operatorname{dim} \sigma}\right)$ to the set of open cells comprising $\overline{e_{\sigma}} \backslash e_{\sigma}$. Since $K$ is a $\mathrm{CW}$ complex in which the closure of each cell is a union of cells, $f_{\sigma}$ must be continuous and surjective onto a union of lower dimensional cells, leaving us to prove injectivity of $f_{\sigma}$ and continuity of $f_{\sigma}^{-1}$. However, once we prove injectivity, we may use the fact that any bijective, continuous map from a compact set to a Hausdorff space is a homeomorphism to conclude continuity of the inverse, so it suffices to prove injectivity.

If the attaching maps for $K$ were not all injective, then we could choose open cells $e_{\rho}, e_{\sigma}$ with $\operatorname{dim}\left(e_{\sigma}\right)-\operatorname{dim}\left(e_{\rho}\right)$ as small as possible such that $e_{\rho} \in \overline{e_{\sigma}}$ and $f_{\sigma}$ restricted to the preimage of $e_{\rho}$ is not 1-1. Then we could choose a point $z \in e_{\rho}$ with $\left|f_{\sigma}^{-1}(z)\right|=k$ for some $k>1$. By condition 4 , $\operatorname{dim}\left(e_{\sigma}\right)-\operatorname{dim}\left(e_{\rho}\right)$ must be at least 2 . We will now show that the open interval $\left(e_{\rho}, e_{\sigma}\right)$ in the closure poset has at least $k$ connected components, which by condition 3 forces $\left[e_{\rho}, e_{\sigma}\right]$ to have rank exactly two. The point is to show for each point $p_{i} \in f_{\sigma}^{-1}(z)$ that there is an open cell $e_{\tau_{i}} \subseteq \overline{e_{\sigma}}$ such that $p_{i} \in \overline{\iota\left(B^{\operatorname{dim} e_{\tau_{i}}}\right)}$, and then to show for distinct points $p_{i}, p_{j} \in f_{\sigma}^{-1}(z)$ that the open cells $e_{\tau_{i}}, e_{\tau_{j}}$ are incomparable in the closure poset. To prove the first part, take an infinite sequence of smaller and smaller balls about $p_{i}$, which by condition 2 must each intersect $f_{\sigma}^{-1}\left(e_{\tau}\right)$ for some $e_{\tau}<e_{\sigma}$ with $\operatorname{dim} e_{\sigma}-\operatorname{dim} e_{\tau}=1$; by finiteness of $K$, the preimage of some such $e_{\tau_{i}}$ is hit infinitely often, implying $p_{i} \in \overline{f_{\sigma}^{-1}\left(e_{\tau_{i}}\right)}$, hence $e_{\rho} \subseteq \overline{e_{\tau_{i}}}$. We prove next that the collections of cells whose closures contain the various points in $f_{\sigma}^{-1}(z)$ must belong to distinct components of $\left(e_{\rho}, e_{\sigma}\right)$, yielding the desired $k$ components in the open poset interval. Consider $p_{1} \neq p_{2}$ with $p_{i} \in \overline{f_{\sigma}^{-1}\left(e_{\tau_{i}}\right)}$ for $i=1,2$. If $e_{\tau_{i}}<e_{\tau_{j}}$ in the closure poset for $\{i, j\}=\{1,2\}$, then condition 5 would imply $\overline{f_{\sigma}^{-1}\left(e_{\tau_{i}}\right)} \subseteq \overline{f_{\sigma}^{-1}\left(e_{\tau_{j}}\right)}$, and hence $p_{1}, p_{2} \in \overline{f_{\sigma}^{-1}\left(e_{\tau_{j}}\right)}$, contradicting the fact that $f_{\tau_{j}}$ restricted to the preimage of $\rho$ is a homeomorphism. Thus, $\left(e_{\rho}, e_{\sigma}\right)$ has no comparabilities between cells whose preimages under $f_{\sigma}$ have closures containing distinct points of $f^{-1}(z)$; in particular, $\left(e_{\rho}, e_{\sigma}\right)$ has at least $k$ connected components, hence must be rank two. 
Finally, we show that $\left(e_{\rho}, e_{\tau}\right)$ has at least $2 k$ elements, forcing $k$ to be 1 , by the thinness requirement in condition 3 . This will contradict our assumption that $k$ was strictly larger than 1 . Lemma 3.8 provides the desired $2 k$ elements by showing that for each of the $k$ preimages of $z$, there are at least two open cells $e_{\tau}$ in $\left(e_{\rho}, e_{\sigma}\right)$ with $\overline{f_{\sigma}^{-1}\left(e_{\tau}\right)}$ containing that particular preimage of $z$.

Lemma 3.8 If a $C W$ complex $K$ meets the conditions of Theorem 1.3, then it also satisfies the following condition: for each open cell $e_{\tau}$ and each $x \in$ $\overline{e_{\tau}} \backslash e_{\tau}$ with $f_{\tau}(x)$ in an open cell $e_{\rho} \subseteq \overline{e_{\tau}}$ with $\operatorname{dim} e_{\tau}-\operatorname{dim} e_{\rho}=2$, there exist distinct open cells $e_{\sigma_{1}}, e_{\sigma_{2}}$ with $\operatorname{dim} e_{\sigma_{i}}=1+\operatorname{dim} e_{\rho}$ and $x \in \overline{f_{\tau}^{-1}\left(e_{\sigma_{i}}\right)}$ for $i=1,2$.

Proof Condition 2 ensures that the boundary of $B^{\operatorname{dim} e_{\tau}}$ does not include any open ( $\left.\operatorname{dim} e_{\tau}-1\right)$-ball, all of whose points map are mapped by $f_{\tau}$ into $e_{\rho}$. In particular, each such ball containing $x$ includes points not sent by $f_{\tau}$ to $e_{\rho}$. Since $K$ is finite, there must be some particular cell $e_{\sigma_{1}}$ such that points arbitrarily close to $x$ within the boundary of $B^{\operatorname{dim} \tau}$ map into $e_{\sigma_{1}}$, implying $x \in \overline{e_{\sigma_{1}}}$, with $\operatorname{dim} e_{\rho}<\operatorname{dim} e_{\sigma_{1}}<\operatorname{dim} e_{\tau}$. Thus, $e_{\rho} \subseteq \overline{{e_{\sigma_{1}}}_{1}}$ and $\operatorname{dim} e_{\sigma_{1}}=$ $\operatorname{dim} e_{\rho}+1$, just as needed.

Now let us find a suitable $e_{\sigma_{2}}$. Here we use the fact that removing the boundary of $e_{\sigma_{1}}$ from a sufficiently small ball $B^{\operatorname{dim} e_{\tau}-1}$ about $x$ yields a disconnected region, only one of whose components may include points from $e_{\sigma_{1}}$. This forces the existence of the requisite open cell $e_{\sigma_{2}}$ which includes points of the other component and has $x$ in its closure.

We will actually use Theorem 1.3 within the following framework:

Corollary 3.9 Let $K$ be a finite, regular $C W$ complex of dimension $p$ and let $f$ be a continuous function from $K$ to a Hausdorff space L. Suppose that $f$ is a homeomorphism on the interior of each open cell and on the closure of each cell of the $(p-1)$-skeleton of $K$. Then $f(K)$ is a finite $C W$ complex satisfying conditions 1,2 , and 5 of Theorem 1.3 , with the restrictions of $f$ to various closed cells in $K$ serving as the characteristic maps.

Proof The restrictions of $f$ to a collection of closures of cells of the $(p-1)$ skeleton give the characteristic maps needed to prove that the $(p-1)$ skeleton of $f(K)$ is a finite CW complex. Now we use Theorem 2.3 to attach the $p$-cells and deduce that $f(K)$ is a finite $\mathrm{CW}$ complex with characteristic maps given by the various restrictions of $f$.

Conditions 1 and 2 are immediate from our assumptions on $f$. If there are two open cells $\sigma_{1}, \sigma_{2}$ in $K$ (of dimension at most $p-1$ ) with identical image under $f$, then the fact that $\bar{\sigma}_{1}$ and $\bar{\sigma}_{2}$ are both regular with isomorphic closure posets gives a homeomorphism from $\sigma_{1}$ to $\sigma_{2}$ preserving cell structure, 
namely the map sending each $x$ to the unique $y$ with $f(y)=f(x)$. This allows us to use the embedding of either $\bar{\sigma}_{1}$ or $\bar{\sigma}_{2}$ in the closure of any higher cell of $K$ to deduce Condition 5. We use that $L$ is Hausdorff and that we have finitely many cells to deduce requirements (1) and (3) of CW complexes.

Although the requirements of Corollary 3.9 may seem quite demanding, Corollary 3.9 is well-suited to proving for a family of regular CW complexes that their images under $f$ are also regular $\mathrm{CW}$ complexes by an induction on dimension. We will use Corollary 3.9 in our main application in the proof of Theorem 6.34, a key inductive result.

\section{Topological collapsing lemmas}

In this section, we introduce certain types of collapses that may be performed sequentially on a convex polytope, and we prove that these preserve homeomorphism type as well as the property of being a finite regular CW complex, though they do not preserve polytopality. In Theorem 4.21, we explicitly define the maps accomplishing these collapses. Then we give a relaxation of the requirements of Theorem 4.21 so as to enable the transfer of a parametrization function on curves in a closed cell $\sigma$ meeting the requirements of Theorem 4.21 to a closed cell with the same cell structure in a different, homeomorphic regular $\mathrm{CW}$ complex which does not necessarily have the same cell structure outside of $\sigma$.

In preparation for these results, we first introduce some helpful properties a topological space or a map may have, with the commonality that in practice these properties may be verified using primarily combinatorics. In what follows, we will typically have a topological space $X$ endowed with the structure of a finite, regular CW complex $K$. We denote this by $X_{K}$ and call $X_{K}$ a regular CW space. Denote by $K /(\operatorname{ker} g)$ the quotient space obtained from an identification map $g$ on a topological space $K$ by setting $x \sim y$ iff $g(x)=g(y)$.

We begin by establishing the following convenient general notion of collapse which is much in the spirit of the concept of elementary collapse, but in fact will preserve homeomorphism type rather than just homotopy type. This section will then be devoted to developing a specific set of checkable conditions which will yield such a collapse.

Definition 4.1 Given a finite regular CW complex $K$ on a set $X$ and an open cell $L$ in $K$, define a face collapse or cell collapse of $\bar{L}$ onto $\bar{\tau}$ for $\tau$ an open cell contained in $\partial L$ to be an identification map $g: X \rightarrow X$ such that:

(1) Each open cell of $\bar{L}$ is mapped surjectively onto an open cell of $\bar{\tau}$ with $L$ mapped onto $\tau$. 
(2) $g$ restricts to a homeomorphism from $K \backslash \bar{L}$ to $K \backslash \bar{\tau}$ and acts homeomorphically on $\bar{\tau}$.

(3) The images under $g$ of the cells of $K$ form a regular CW complex with new characteristic maps obtained by composing the original characteristic maps of $K$ with $g^{-1}: X_{K} \rightarrow X_{K}$ for those cells of $K$ contained either in $\bar{\tau}$ or in $(K \backslash \bar{L})$.

We call such a map $g$ a collapsing map. Remark 4.2 will show that these collapses preserve homeomorphism type.

The collapse of a cell $L$ often will also collapse other cells in its closure in the process.

Remark 4.2 The induced map $\bar{g}: X_{K} /(\operatorname{ker} g) \rightarrow X$ is continuous by Remark 2.5, and it is bijective by how it is defined. Since $K$ is Hausdorff and $K /(\operatorname{ker} g)$ is compact, we may conclude that $\bar{g}$ is a homeomorphism. Thus, face collapses preserve homeomorphism type.

Remark 4.3 If $g$ is a collapsing map on $X_{K}$, and $h$ is a homeomorphism from the underlying space $X$ to itself, then $g \circ h$, composing functions right to left, is a collapsing map as well.

For example, Remark 4.3 may enable a change of coordinates for convenience prior to a collapse. The following may also be helpful for controlling how such a homeomorphism such as a change of coordinates map may interact with subsequent cell collapses.

Lemma 4.4 Suppose $K_{1}$ and $K_{2}$ are topological spaces, $f$ is a homeomorphism from $K_{1}$ to $K_{2}, \pi_{1}: K_{1} \rightarrow K_{1}$ and $\pi_{2}: K_{2} \rightarrow K_{2}$ are identification maps giving rise to quotient spaces $K_{1} / \sim$ and $K_{2} / \sim^{\prime}$. If we also have $x \sim y$ in $K_{1}$ iff $f(x) \sim^{\prime} f(y)$ in $K_{2}$, then $K_{1} / \sim$ is homeomorphic to $K_{2} / \sim^{\prime}$ under the induced map $\bar{f}$.

Proof This follows easily from Proposition 13.5 in Chap. 1 of [7] by constructing a suitable commutative diagram.

Definition 4.5 Given a collection of parallel line segments $\mathcal{C}$ covering a face $F$ of a polytope $P$, define a length function len $F \rightarrow \mathbb{R}$ by letting len $(x)$ be the length of the element of $\mathcal{C}$ containing $x$. Now define a parametrization $p: F \rightarrow[0,1]$ by letting the restriction of $p$ to any $c \in \mathcal{C}$ be the linear function from $c$ to $[0,1]$.

Remark 4.6 Convexity of $P$ implies len is continuous, which in turn implies that $p$ is also continuous on $\operatorname{int}(F)$ and everywhere on $F$ except at points 
comprising parallel line segments consisting of just single points, though here nearby parallel line segments also approach length 0 . Moreover, if $h$ is any homeomorphism from $\operatorname{int}(F)$ to another topological space, then $p \circ h^{-1}$ is also continuous.

Continuity of len will allow us to perform collapses across families of parallel-like curves, as defined next, according to a continuous function that stretches a collar to cover a face being collapsed. This stretching function is provided in Theorem 4.21.

Definition 4.7 Let $K_{0}$ be a convex polytope, and let $\mathcal{C}_{i}^{0}$ be a family of parallel line segments covering a closed face $L_{i}^{0}$ in $\partial K_{0}$ with the elements of $\mathcal{C}_{i}^{0}$ given by linear functions $c:[0,1] \rightarrow L_{i}^{0}$. Suppose that there is a pair of closed faces $G_{1}, G_{2}$ in $\partial L_{i}^{0}$ with $c(0) \in G_{1}$ and $c(1) \in G_{2}$ for each $c \in \mathcal{C}_{i}^{0}$ and there is a composition $g_{i} \circ \cdots \circ g_{1}$ of face collapses (cf. Definition 4.1) on $K_{0}$ such that:

(1) $g_{i} \circ \cdots \circ g_{1}$ acts homeomorphically on $\operatorname{int}\left(L_{i}^{0}\right)$.

(2) For each $c \in \mathcal{C}_{i}^{0}, g_{i} \circ \cdots \circ g_{1}$ either sends $c$ to a single point or acts homeomorphically on $c$.

(3) Suppose $g_{i} \circ \cdots \circ g_{1}(c(t))=g_{i} \circ \cdots \circ g_{1}\left(c^{\prime}\left(t^{\prime}\right)\right)$ for $c \neq c^{\prime} \in \mathcal{C}_{i}^{0}$ and some $\left(t, t^{\prime}\right) \neq(1,1)$. Then $t=t^{\prime}$, and for each $t \in[0,1]$ we have $g_{i} \circ \cdots \circ$ $g_{1}(c(t))=g_{i} \circ \cdots \circ g_{1}\left(c^{\prime}(t)\right)$.

Then call $\mathcal{C}_{i}=\left\{g_{i} \circ \cdots \circ g_{1}(c) \mid c \in \mathcal{C}_{i}^{0}\right\}$ a family of parallel-like curves on the closed cell $L_{i}=g_{i} \circ \cdots \circ g_{1}\left(L_{i}^{0}\right)$ of the finite regular CW complex $K_{i}=$ $g_{i} \circ \cdots \circ g_{1}\left(K_{0}\right)$.

Remark 4.8 These conditions are designed so that they only need to be checked just prior to the $k$-th collapsing step for the curves covering a cell to be collapsed in the $k$-th collapse.

Notice that Definition 4.7, part (3), implies the curves are nonoverlapping except perhaps at their endpoints in $g_{i} \circ \cdots \circ g_{1}\left(G_{2}\right)$. Verifying (3) mainly requires showing curves have distinct endpoints in $g_{i} \circ \cdots \circ g_{1}\left(G_{1}\right)$, leading to the following:

Condition 4.9 Let us call Definition 4.7, part (3), the distinct initial points condition (DIP).

Remark 4.10 In practice, we will verify Definition 4.7, part (2), by verifying Condition 4.11 below; this suffices because $g_{i} \circ \cdots \circ g_{1}$ acts homeomorphically on each open cell not collapsed by any $g_{j}$ for $j \leq i$. 
We say that a curve is nontrivial if it includes more than one point.

Condition 4.11 A collection $\mathcal{C}$ of curves covering the closure of a cell $F$ satisfies the distinct endpoints condition (DE) if for any nontrivial curve $c \in \mathcal{C}$, the two endpoints of $c$ live in distinct cells in $\bar{F}$.

The fact that each collapse restricts to a homeomorphism on the interior of each cell it does not collapse allows us to reduce what actually must be checked to show that a family of curves is parallel-like to the following:

Remark 4.12 To verify the requirements of Definition 4.7 for the family of curves $\mathcal{C}_{i}$ covering a cell $L_{i}$ to be collapsed in the $i$-th collapsing step, it suffices to show:

(1) Each earlier collapse restricts to a homeomorphism on each open cell which is not collapsed prior to the $i$-th collapsing step

(2) (DIP) for $\mathcal{C}_{i}$ holds just prior to the $i$-th collapse

(3) (DE) for $\mathcal{C}_{i}$ holds just prior to the $i$-th collapse

(4) Consistency of parametrizations when entire curves in $\mathcal{C}_{i}$ that are in $\partial L_{i}$ are identified with each other in earlier collapses.

In our main application, and quite possibly in other future applications as well, the last condition will follow immediately from our set-up; more specifically, it will follow from how each collapse replace a pair of parameters $t_{r_{k}-1}^{\prime}$ and $t_{r_{k}}^{\prime}$ by a single parameter equalling their sum. Next we give the ingredients that will allow us to extend our collapsing maps through collars, culminating in Condition 4.17 and Lemma 4.18.

Definition 4.13 Given a continuous, surjective function $g_{i+1}: X \rightarrow X$, define an interpolating family of maps $\left\{g_{i+1, t} \mid t \in[0,1]\right\}$ from $X$ to $X$ as a collection of maps with $g_{i+1,0}=g_{i+1}$ and $g_{i+1,1}=i d$, requiring (1) for each $t \in(0,1]$ that $g_{i+1, t}$ be a homeomorphism from $X$ to $X$ and (2) continuity of the map $h_{i+1}: X \times[0,1] \rightarrow X$ defined by $h_{i+1}(x, t)=g_{i+1, t}(x)$.

Remark 4.14 This notion of interpolating family of maps is almost exactly the topological notion of isotopy, except that our initial map $g_{i+1,0}$ is not a homeomorphism. In our setting, $g_{i+1,0}$ is a collapsing map which will admit approximations by homeomorphisms.

Lemma 4.15 Suppose a collapsing map $g$ collapses across a family of parallel-like curves $\mathcal{C}$ where each $c \in \mathcal{C}$ is sent to itself by a monotonically increasing, piecewise linear function $g:[0,1] \rightarrow[0,1]$. Then $g$ gives rise to an interpolating family of maps. 
Proof By definition, we must have $0=a_{1}<\cdots<a_{k}=1$ and $0=b_{1} \leq \cdots \leq$ $b_{k}=1$ such that $g$ maps $\left[a_{i}, a_{i+1}\right]$ to $\left[b_{i}, b_{i+1}\right]$ by a linear map for each $1 \leq i \leq k-1$. Then define $g_{t}$ instead to map $\left[a_{i}, a_{i+1}\right]$ linearly to $\left[t a_{i}+(1-\right.$ $\left.t) b_{i}, t a_{i+1}+(1-t) b_{i+1}\right]$.

Lemma 4.16 If a collapsing map $g_{i+1}$ on a regular $C W$ space $X_{K}$ homeomorphic to a sphere gives rise to an interpolating family of maps $\left\{g_{i+1, t} \mid t \in\right.$ $[0,1]\}$ on $X_{K}$, then $g_{i+1}$ extends to a collapsing map on any ball $B$ having $X_{K}$ as its boundary, implying $g_{i+1}(B)$ is a regular $C W$ complex homeomorphic to a ball.

Proof Choose a collar $X \times[0,1]$ for $B$, which exists by Theorem 2.8. For each $(x, t) \in X \times[0,1]$, let $g_{i+1}(x, t)=g_{i+1, t}(x)$. Let $g_{i+1}$ act as the identity map on $B \backslash(X \times[0,1])$.

Condition 4.17 A finite regular $\mathrm{CW}$ complex $K$ has the inductive manifold condition (IM) if for each open $d$-cell $\tau$ and each $(d+1)$-cell $\sigma$ such that $\tau \subseteq \partial \sigma, \overline{\partial \sigma \backslash \tau}$ is a compact manifold with boundary.

Next we show how to use collars to extend a collapsing map from the boundary of a low-dimensional closed cell to an entire cell complex.

Lemma 4.18 Let $K$ be a regular $C W$ complex having a unique maximal cell and satisfying Condition 4.17 (IM). Let $\tau$ be an $i$-cell in the boundary of an $(i+1)$-cell $\sigma$. Let $g_{i+1}$ be a collapsing map on $\partial \sigma$ that collapses the cell $\tau$. If $g_{i+1}$ gives rise to an interpolating family of maps, then $g_{i+1}$ may be extended to a collapsing map on $K$.

Proof First choose a series of cells $\tau=\sigma_{1}, \sigma=\sigma_{2}, \sigma_{3}, \ldots, \sigma_{k}$ such that $\sigma_{j} \subseteq$ $\overline{\sigma_{j+1}}$ with $\operatorname{dim} \sigma_{j+1}=\operatorname{dim} \sigma_{j}+1$ for each $j$, letting $\sigma_{k}$ be the unique maximal cell of $K$. We are given $g_{i+1}$ defined on $\partial \sigma$ and will now describe for each $2 \leq j \leq k-1$ how to extend $g_{i+1}$ from $\partial \sigma_{j}$ to $\overline{\sigma_{j}}$ and then to $\partial \sigma_{j+1}$.

Lemma 4.16 enables us to extend $g_{i+1}$ from $\partial \sigma_{j}$ to $\overline{\sigma_{j}}$. If $\sigma_{j}$ is the big cell, we are done. Otherwise, choose $\sigma_{j+1}$ with $\overline{\sigma_{j}} \subseteq \partial \sigma_{j+1}$ and take a collar for $\overline{\partial \sigma_{j+1} \backslash \sigma_{j}}$ within $\partial \sigma_{j+1}$, which exists by Condition 4.17 and Theorem 2.8. Use an interpolating family to extend $g_{i+1}$ from $\overline{\sigma_{j}}$ to $\partial \sigma_{j+1}$, defining the interpolating family as follows.

Assuming $g_{i+1}$ has been defined on the first $r-1$ collars in the above alternation, let $g_{i+1}\left(x, t_{1}, t_{2}, \ldots, t_{r}\right)=\left(g_{i+1,1-\left(1-t_{1}\right) \cdots\left(1-t_{r}\right)}(x), t_{2}, \ldots, t_{r}\right)$ for each $\left(t_{1}, \ldots, t_{r}\right) \in[0,1]^{r}$, and let $g_{i+1}$ act as the identity on all points of $\overline{\sigma_{r}}$ (resp. $\partial \sigma_{r+1} \backslash \overline{\sigma_{r}}$ ) not in our collar for $\overline{\sigma_{r}}$ (resp. $\partial \sigma_{r+1} \backslash \overline{\sigma_{r}}$ ) as well as for points that are in our collar but sit over points that were not in the collar at an earlier stage. 
Now to a condition that will be used to prove that our upcoming collapses preserve regularity:

Condition 4.19 Let $g$ be an identification map on a regular CW space $X_{K}$ such that $g$ maps cells onto cells, maps an open cell $F$ onto one of its boundary cells, and acts homeomorphically on $X_{K} \backslash \bar{F}$. Then $g$ satisfies the least upper bound condition (LUB) if for any pair of open cells $A, B \subseteq \bar{F}$ such that $g(A)=g(B)$ and any face $F^{\prime}$ that is a least upper bound for $A$ and $B$ in the closure poset just prior to the application of $g, F^{\prime}$ is also mapped onto one of its boundary cells by $g$.

Remark 4.20 At each collapsing step, there will be one cell among those getting collapsed that has all other cells getting collapsed in the same step in its closure. (LUB) then implies for any pair of cells $F, F^{\prime}$ which are both least upper bounds for cells $\sigma$ and $\sigma^{\prime}$ just prior to the collapse of $F$ such that this collapse identifies $\sigma$ with $\sigma^{\prime}$, then this step also collapses a larger cell having both $F$ and $F^{\prime}$ in its closure, doing so in a way that induces the collapses of $F$ and $F^{\prime}$.

Next is the main result of this section, a topological construction showing how under certain (mainly combinatorial) conditions a regular CW ball admits a cell collapse (in the sense of Definition 4.1), hence admits an identification map preserving homeomorphism type and regularity. The result is phrased as an inductive statement so as to allow the performance of a series of such collapses by showing that after each collapse the conditions are preserved that are needed to apply the theorem again. The proof is largely devoted to defining explicitly a suitable continuous, surjective map based on a collection of parallel-like curves covering the cell to be collapsed. Figures 1 and 2 provide pictures that may be helpful to seeing what these maps are doing.

Theorem 4.21 Let $K_{0}$ be a convex polytope. Let $g_{1}, \ldots, g_{i}$ be collapsing maps with $g_{j}: X_{K_{j-1}} \rightarrow X_{K_{j}}$ for regular $C W$ complexes $K_{0}, \ldots, K_{i}$ all having underlying space $X$. Suppose $K_{i}$ satisfies Condition 4.17 (IM) and that there is an open cell $L_{i}^{0}$ in $\partial K_{0}$ upon which $g_{i} \circ \cdots \circ g_{1}$ acts homeomorphically and a collection $\mathcal{C}=\left\{g_{i} \circ \cdots \circ g_{1}(c) \mid c \in \mathcal{C}_{i}^{0}\right\}$ of parallel-like curves covering $\overline{L_{i}}$ for $L_{i}=g_{i} \circ \cdots \circ g_{1}\left(L_{i}^{0}\right) \in K_{i}$. Then there is an identification map $g_{i+1}: X_{K_{i}} \rightarrow X_{K_{i+1}}$ specified by $\mathcal{C}$. If $g_{i+1}$ also satisfies Condition 4.19 $(L U B)$, then $g_{i+1}$ is a collapsing map and $K_{i+1}$ is a regular $C W$ complex also satisfying Condition 4.17 (IM).

Proof We may assume $L_{i}$ is top dimensional in $\partial K_{i}$, because otherwise we may choose a cell $L^{\prime}$ in $K_{i}$ with $\operatorname{dim} L^{\prime}=\operatorname{dim} L_{i}+1$ and $L_{i} \subseteq \partial L^{\prime}$, define the 
Fig. 1 Schematic for collapsing map

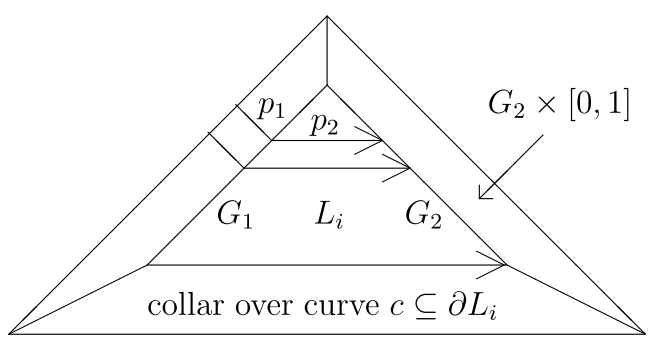

collapsing map on $\partial L^{\prime}$ as described below, then use Lemma 4.18 to extend $g_{i+1}$ to the entire complex. We will define $g_{i+1}$ on $\partial L^{\prime}$ in such a way that Lemma 4.16 will enable its extension to $\bar{L}^{\prime}$.

We will construct a continuous, surjective function $g_{i+1}$ that maps entire curves in $\mathcal{C}$ to points in $G_{2}$ (cf. Definition 4.7), thereby collapsing $\overline{L_{i}}$ onto $G_{2}$, and in the process identifying each point of $G_{1}$ with a point of $G_{2} ; g_{i+1}$ restricted to $K_{i} \backslash \overline{L_{i}}$ will be a homeomorphism. First we define an auxiliary family $\mathcal{C}^{\prime}$ of curves that covers not only $\overline{L_{i}}$ but also a collar just outside its boundary. We will stretch these curve extensions from the collar to cover $\overline{L_{i}}$; the introduction of additional curves within the collar will enable interpolation from the action of $g_{i+1}$ on $\overline{L_{i}}$ to the identity map outside this collar. Now to the details.

First consider any $c \in \mathcal{C}$ with $c \cap \partial\left(L_{i}\right)=\{x, y\}$ for points $x \in \overline{G_{1}}$ and $y \in \overline{G_{2}}$. Extend $c$ to include all points $(y, t)$ and $(x, t)$ for $t \in[0,1]$ to obtain a lengthened curve $c^{\prime}$. Since $c_{1} \neq c_{2}$ for $c_{1}, c_{2} \in \mathcal{C}$ implies that $c_{1}$ and $c_{2}$ have distinct endpoints in $G_{1}$ by Definition 4.7, part 3, the curve extensions $\{(x, t) \mid t \in[0,1]\}$ given by the various points $x \in G_{1}$ are nonoverlapping. It will not matter if distinct $c_{1}, c_{2} \in \mathcal{C}$ have the same endpoint $y \in G_{2}$, because $\left.g_{i+1}\right|_{G_{2} \times[0,1]}=I D$. In this situation, let $y \times[0,1]$ be part of both $c_{1}^{\prime}$ and $c_{2}^{\prime}$. Definition 4.7, part 2, guarantees $x \neq y$ for each nontrivial $c \in \mathcal{C}$. For each $c \in \mathcal{C}$ where $c$ is a single point in $\partial\left(L_{i}\right)$, extend to $c^{\prime}=\{(c, t) \mid t \in[0,1]\}$, and let $\left.g_{i+1}\right|_{c^{\prime}}=I D$. For each nontrivial curve $c$ with $c \subseteq \partial\left(L_{i}\right)$, we create a family $F_{c}$ of curves in $N_{i}$ (see Fig. 2) so that $F_{c}$ covers exactly $\{c\} \times[0,1]=\{(x, t) \mid x \in c ; t \in[0,1]\}$. We make one such curve $c_{t} \in F_{c}$ for each $t \in[0,1]$, doing this in such a way that we have $c \subseteq c_{0}$. Letting $c=\{c(t) \mid t \in[0,1]\}$ with $c(0) \in G_{1}$ and $c(1) \in G_{2}$, then for each $t \in[0,1]$, we define $c_{t}$ as $\left\{\left(c(t / 2), t^{\prime}\right) \mid t^{\prime} \geq t\right\} \cup\left\{\left(c\left(t^{\prime \prime}\right), t\right) \mid t^{\prime \prime} \geq t / 2\right\} \subseteq\{c\} \times[0,1]$. Now $\mathcal{C}^{\prime}$ is comprised of the union of these families of curves $F_{c}$ for each nontrivial curve $c \subseteq \partial L_{i}$, along with an extended curve $c^{\prime}$ resulting from each $c \in \mathcal{C}$ which is trivial or only intersects $\partial\left(L_{i}\right)$ in its endpoints.

Now we define $g_{i+1}: K_{i} \rightarrow K_{i}$ by specifying how it maps each $c^{\prime} \in \mathcal{C}^{\prime}$ surjectively onto itself. First consider any $c^{\prime} \in \mathcal{C}^{\prime}$ obtained by extending some nontrivial $c \in \mathcal{C}$. Represent the points of $c^{\prime}$ as $\{c(t) \mid t \in[-1,2]\}$, where $[-1,0]$ gives $p_{1}=c^{\prime} \cap\left(G_{1} \times[0,1]\right)$, i.e. the part of the collar sitting over the 
Fig. 2 Defining $g_{i+1}$ on collar portion over curve $c \subseteq \partial L_{i}$

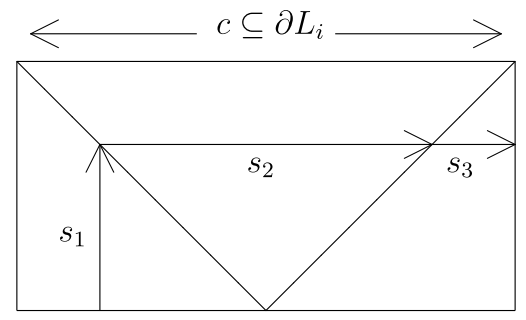

$\partial L_{i} \times\{0\}$

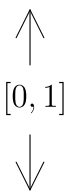

$\partial L_{i} \times\{1\}$

endpoint of $c$ in $G_{1}$, whereas $[0,1]$ specifies the points in $p_{2}=c^{\prime} \cap L_{i}$, and $[1,2]$ gives the points in $p_{3}=c^{\prime} \cap\left(G_{2} \times[0,1]\right)$. Let $g_{i+1}(c(t))=c(1) \in \overline{G_{2}}$ for $t \in[0,1]$, i.e. let $g_{i+1}$ map the entire segment $p_{2}$ in Fig. 1 to its endpoint in $\overline{G_{2}}$; let $g_{i+1}(c(t))=c(2 t+1)$ for $t \in[-1,0]$, i.e. stretch the segment $p_{1}$ in Fig. 1 to cover $p_{1} \cup p_{2}$; and let $g_{i+1}(c(t))=c(t)$ for $t \in[1,2]$.

Next consider any family $F_{c}$ of elements of $\mathcal{C}^{\prime}$ covering $c \times[0,1]$ for some $c \in \mathcal{C}$ with $c \subseteq \partial\left(L_{i}\right)$. The map here is designed so as to interpolate from the collapsing map needed in $\partial L_{i}$ to the identity map outside the collar. Points are represented as ordered pairs $\left(c\left(t_{1}\right), t_{2}\right)$ for $t_{1}, t_{2} \in[0,1]$. For each $t \in[0,1]$, the map $g_{i+1}$ sends $s_{2} \cup s_{3}=\left\{\left(c\left(t^{\prime}\right), t\right) \mid t / 2 \leq t^{\prime} \leq 1\right\}$ to $s_{3}=\left\{\left(c\left(t^{\prime}\right), t\right) \mid 1-\right.$ $\left.t / 2 \leq t^{\prime} \leq 1\right\}$ by appropriate scaling of the parameter, and $g_{i+1}$ stretches $s_{1}=$ $\left\{\left(c(t / 2), t^{\prime}\right) \mid t^{\prime} \geq t / 2\right\}$ to cover $s_{1} \cup s_{2}$ for $s_{2}=\left\{\left(c\left(t^{\prime}\right), t\right) \mid t / 2 \leq t^{\prime} \leq 1-t / 2\right\}$, again by reparametrization by a suitable linear scaling factor. See Fig. 2. In other words, $g_{i+1}$ sends $\left\{\left(c(t / 2), t^{\prime}\right) \mid \frac{1+t}{2} \leq t^{\prime} \leq 1\right\}$ to $\left\{\left(c(t / 2), t^{\prime}\right) \mid t \leq t^{\prime} \leq 1\right\}$ and sends $\left\{\left(c(t / 2), t^{\prime}\right) \mid t \leq t^{\prime} \leq \frac{1+t}{2}\right\}$ to $\left\{\left(c\left(t^{\prime}\right), t\right) \mid t / 2 \leq t^{\prime} \leq 1-t / 2\right\}$.

Note that $g_{i+1}$ acts as the identity on $\partial\left(N_{i}\right)$ and acts injectively on $N_{i} \backslash \overline{L_{i}}$. We describe next how to choose the parametrizations of various curves in $\mathcal{C}^{\prime}$ in a way that makes $g_{i+1}$ continuous. We use that the relative interiors of the curves in $\mathcal{C}$ have preimages that are a family of parallel line segments covering a convex region (a face of a polytope), enabling us to choose parametrizations within this simplex which induce suitable ones for $\mathcal{C}$; the point is to use $\operatorname{Re}-$ mark 4.5 to give a continuous function from $L_{0}^{i}$ to $[0,1]$ recording for each point how it is parametrized within a curve in $\mathcal{C}$. From this, we obtain suitable parametrizations for $\mathcal{C}^{\prime}$ by virtue of the collar that is also covered by $\mathcal{C}^{\prime}$ being homeomorphic to $\partial\left(L_{i}\right) \times[0,1]$.

Surjectivity and continuity of $g_{i+1}$ imply it induces a continuous, bijective function $\overline{g_{i+1}}$ from $K_{i} /\left(\operatorname{ker} g_{i+1}\right)$ to $K_{i+1}$. Continuity of $\left(\overline{g_{i+1}}\right)^{-1}$ is then immediate, because any bijective, continuous function from a compact set to a Hausdorff space has continuous inverse. Thus, $g_{i+1}\left(X_{K_{i}}\right)=X_{K_{i+1}}$ is homeomorphic to $X_{K_{i}} /\left(\operatorname{ker} g_{i+1}\right)$ via $\overline{g_{i+1}}$. It is straightforward to see that the equivalence relation under $\operatorname{ker} g_{i+1}$ gives a closed subset of $X_{k} \times X_{k}$, implying $X_{K_{i}} /\left(\operatorname{ker} g_{i+1}\right)$ is Hausdorff.

Now let us check that regularity is also preserved under $g_{i+1}$. Condition 4.19 (LUB) implies for any cell $G$ not collapsed by $g_{i+1} \circ \cdots \circ g_{1}$ that 
any cells $\sigma_{1}, \sigma_{2} \subseteq \partial(G)$ identified by $g_{i+1}$ must have some least upper bound $A \subseteq \partial(G)$ which is also collapsed by $g_{i+1}$. Thus, our homeomorphism $\overline{g_{i+1}}$ will restrict to $\bar{G} /\left(\operatorname{ker} g_{i+1}\right)$, enabling us to define the attaching map for $G$ as a composition of three maps, first applying the attaching map for $G$ within $K_{i}$, then composing this with $\left.g_{i+1}^{-1}\right|_{\bar{G}_{\text {final }}}$, regarded as a map from $X_{K_{i}}$ to $X_{K_{i}}$, then composing with $g_{i+1}: X_{K_{i}} \rightarrow X_{K_{i+1}}$, the point of the second map being to send points the cells of $K_{i}$ to the cells of $K_{i+1}$; here we let $\bar{G}_{\text {final }}$ denote the set of cells mapped homeomorphically to themselves by $g_{i+1}$ or in other words the cells that the various fibers of $g_{i+1}$ are mapped onto. Lemmas 4.15 and 4.16 show that this may be extended to yield a characteristic map for all of $\bar{G}$, making it a regular CW complex homeomorphic to a ball.

Lemma 4.22 will verify Condition 4.17 (IM) for $K_{i+1}$.

Lemma 4.22 Collapses as in Theorem 4.21 preserve Condition 4.17, i.e. the inductive manifold condition.

Proof Consider any pair of cells $\tau \subseteq \bar{\sigma}$ in $K_{i+1}$ with $\operatorname{dim} \sigma=\operatorname{dim} \tau+1$. We must show that $\overline{\partial \sigma \backslash \tau}$ is a compact manifold with boundary. By our definition of collapsing map, there must be cells $\tau_{i}, \sigma_{i}$ in $K_{i}$ with $g_{i+1}$ mapping $\tau_{i}$ homeomorphically to $\tau$ and $\sigma_{i}$ homeomorphically to $\sigma$ with $\tau_{i} \subseteq \overline{\sigma_{i}}$. The proof of Theorem 4.21 shows that $\overline{g_{i+1}}$ is a homeomorphism from $\overline{\sigma_{i}} /\left(\operatorname{ker} g_{i+1}\right)$ to $\bar{\sigma}$. In particular, this implies that $\overline{g_{i+1}}$ gives a bijection from $\left(\overline{\partial \sigma_{i} \backslash \tau_{i}}\right) /\left(\operatorname{ker} g_{i+1}\right)$ to $\overline{\partial \sigma \backslash \tau}$. By definition, $\overline{g_{i+1}}$ is continuous and $\operatorname{ker}\left(\overline{g_{i+1}}\right)=\left\{\left(x, x^{\prime}\right) \mid \overline{g_{i+1}}(x)=\overline{g_{i+1}}\left(x^{\prime}\right)\right\} \subseteq K_{i} \times K_{i}$ is closed, implying that $\overline{g_{i+1}}\left(\left(\overline{\partial \sigma_{i} \backslash \tau_{i}}\right) /\left(\operatorname{ker} g_{i+1}\right)\right)$ is compact and Hausdorff by Proposition 13.8 in Chap. 1 of [7]. Since $\overline{g_{i+1}}$ is a continuous, bijective map from a compact set to a Hausdorff space, $\overline{g_{i+1}}$ also has continuous inverse. Hence, the property of being a compact manifold with boundary transfers as desired.

Our collapsing map as defined in the proof of Theorem 4.21 is defined in terms of curve parametrizations and their extensions across collars. Polytopality of $K_{0}$ is only used to supply such a parametrization function that is continuous. Thus, Theorem 4.21, Lemma 4.22, and Proposition 4.26 all hold in more generality than how they are stated, without requiring any modifications to their proofs, yielding:

Corollary 4.23 In Theorem 4.21, we may replace the polytope $K_{0}$ by any regular $C W$ complex satisfying the inductive manifold condition and replace the parallel line segments covering a face $L_{i}^{0}$ by any family $\mathcal{C}_{0}$ of curves covering a closed cell $L_{i}^{0}$ such that (1) all of the curves have one endpoint living in a closed cell $\bar{G}_{1} \subseteq \partial\left(L_{i}^{0}\right)$ and the other endpoint in a closed cell $\bar{G}_{2} \subseteq \partial\left(L_{i}^{0}\right)$, (2) these curves in $\mathcal{C}_{0}$ are nonoverlapping except possibly at their endpoints in $\bar{G}_{1}$, and (3) there is a continuous function $p$ from 
$L_{i}^{0} \backslash\left\{x \in L_{i}^{0} \mid x\right.$ comprises a trivial curve in $\left.\mathcal{C}_{0}\right\}$ to $[0,1]$ that restricts to a homeomorphism from each nontrivial curve $c \in \mathcal{C}_{0}$ to $[0,1]$.

An especially useful special case is the following, which in our main application will allow us to incorporate a change of coordinates homeomorphism $c h$ which changes the reduced word with respect to which we work and hence may change the cell structure outside of the closed cell being collapsed:

Corollary 4.24 Let $K$ be a regular $C W$ complex with closed cell $L$ covered by a family of curves $\mathcal{C}$ and let $\phi$ be a homeomorphism from $K$ to a regular $C W$ complex $K^{\prime}$ with closed cell $L^{\prime}$ covered by a family $\mathcal{C}^{\prime}$ of parallel-like curves such that $\phi$ restricted to $L$ is a cell-structure preserving homeomorphism to $L^{\prime}$ mapping each curve in $\mathcal{C}$ to a curve in $\mathcal{C}^{\prime}$. Then we may transfer the parametrization function for $L^{\prime}$ to one for $L$, enabling the collapse of $L$ across the curves in $\mathcal{C}$ via exactly the collapsing map given in the proof of Theorem 4.21. Specifically, it does not matter if $K$ has an entirely different cell structure from $K^{\prime}$ outside of the closed cell $L$.

Next we turn to the links of the cells. Following [11], we use (essentially) the notion of link in the sense of Whitney stratified spaces (as defined in [14]) for a series of quotient cell complexes obtained by repeated application of Theorem 4.21 and its extension in Corollary 4.24. In the following proposition, we use a polyhedral cone $\mathcal{C}$ whose cross-sectional slices are copies of our polytope, as well as using the quotient spaces of $\mathcal{C}$ under our collapses, denoted $\mathcal{C} / \sim_{k}$. We assume that for each $\tau \subseteq \bar{\sigma}$ we have a projection map $\pi_{\tau}^{\sigma}$ onto the region $R_{\tau}$ of $\mathcal{C} / \sim_{k}$ indexed by the cell $\tau$. We require that the inverse image under $\pi_{\tau}^{\sigma}$ of each point $p \in R_{\tau}$ lives in a transversal to the open cell $\tau$ where $\tau$ is obtained by restricting $R_{\tau}$ to the cross-sectional slice of $\mathcal{C} / \sim$ containing $p$; moreover, we require that this transversal also gives transversals through sufficiently nearby cross-sectional slices farther from the origin as well. The map $\pi_{\tau}^{\sigma}$ is defined to have as its domain the intersection of the following two sets $S_{1}$ and $S_{2}$ : (1) $S_{1}$ is the part of $\mathcal{C} / \sim_{k}$ consisting of the cross-sectional slice containing $p$ as well as all slices farther from the origin, while (2) $S_{2}$ is the set of regions of $\mathcal{C} / \sim_{k}$ indexed by the cells in $\bar{\sigma}$ having $\tau$ in their closure.

Definition 4.25 Take a point $p$ in the interior of $\tau$ (in any chosen slice of $\mathcal{C} / \sim_{k}$ besides the origin). Define the link of $\tau$ in $\bar{\sigma}$, denoted $l k(\tau, \sigma)$, as the intersection of the set $\left(\pi_{\tau}^{\sigma}\right)^{-1}(p)$ with a sufficiently nearby cross-sectional slice of $\mathcal{C} / \sim_{k}$ contained in $S_{1}$ and not containing $p$.

Proposition 4.26 Suppose a cell collapse of $L_{i}$ which meets the conditions of Theorem 4.21 or Corollary 4.24 also satisfies $\operatorname{dim}\left(G_{1}\right)=\operatorname{dim}\left(G_{2}\right)=$ 
$\operatorname{dim}\left(L_{i}\right)-1$. Suppose additionally that there are projection maps $\pi_{\tau}^{\sigma}$ giving rise to links as defined above both after the current collapsing step and at all earlier steps. Also suppose that the preimage under the current collapse of each transversal is the closure of a disjoint union of such transversals given by the corresponding projection maps on the preimage restricted to int $\left(L_{i}\right)$ and that this closure is contained in the disjoint union of the transversals for the projection maps on $\overline{L_{i}}$. Then the collapse will preserve the property that the link of each cell is a closed ball with induced cell decomposition a regular $\mathrm{CW}$ decomposition.

Proof Let $F_{i+1}$ be the maximal cell collapsed at this step, and let $g_{i+1}$ be the collapsing map. The result is immediate for cells whose links (prior to the collapse) do not intersect $\bar{F}_{i+1}$, since $g_{i+1}$ acts homeomorphically everywhere except on $\bar{F}_{i+1}$, leaving such links unchanged.

Letting $\bar{G}_{2}$ be the closed cell onto which $g_{i+1}$ maps $\bar{F}_{i+1}$, we now check the result for the link of the open cell $G_{2}$. Let $\bar{G}_{1}$ be the closed cell containing the other endpoints of the parallel-like curves across which $\bar{F}_{i+1}$ is collapsed. The point is to show that $g_{i+1}^{-1}(l k(p))$ for $p \in G_{2}$ is a ball and that homeomorphism type is preserved under application of $g_{i+1}$. Our use of parallel-like curves allows us to decompose $g_{i+1}^{-1}(l k(p))$ into three pieces, namely its restrictions to (a) points sitting over $\bar{G}_{1}$, (b) points sitting over $\bar{G}_{2}$ and (c) points sitting over $\bar{F}_{i+1} \backslash\left(\bar{G}_{1} \cup \bar{G}_{2}\right)$. The fact that earlier collapses were performed successfully yielding links that were regular $\mathrm{CW}$ balls implies that (a) and (c) are each regular CW balls and that (b) is the product of a regular $\mathrm{CW}$ ball with the open interval $(0,1)$. The parallel-like curves glue these together in a natural way that makes the union also a regular $\mathrm{CW}$ ball, since (b) is $(0,1) \times l k_{\sigma / \sim_{i}} F_{i+1}$ where the desired link is being taken in $\sigma$ and $\sim_{i}$ is the equivalence relation resulting from the first $i$ collapses, while (a) and (c) are each homeomorphic to $l k_{\sigma / \sim_{i}} F_{i+1}$. Moreover, applying $g_{i+1}$ glues the ball sitting over $\bar{G}_{2}$ to the ball sitting over $\bar{G}_{1}$ by identifying the endpoints of curves sitting over $\bar{F}_{i+1} \backslash\left(\bar{G}_{1} \cup \bar{G}_{2}\right)$, yielding a ball. This would be more subtle without our assumption that $\operatorname{dim}\left(G_{1}\right)=\operatorname{dim}\left(G_{2}\right)$.

The same approach applies to the link of any open cell contained in $\bar{G}_{2}$ which likewise is not collapsed by $g_{i+1}$ but also has some cell of dimension one higher than it collapsed onto it by $g_{i+1}$. Finally, consider the link of any cell $\sigma$ that is not collapsed by $g_{i+1}$, but is in the closure of a cell that is collapsed by $g_{i+1}$; the only remaining such case is for a face $\sigma$ that is covered by parallel-like curves that are each just a single point. In this case, the result follows from the fact that our homeomorphism from $X /\left(\operatorname{ker} g_{i+1}\right)$ to $X$ naturally restricts to some neighborhood of any point in $\sigma$ by choosing a neighborhood whose boundary is a union of points which are fixed by $g_{i+1}$ together with entire curves from our family of parallel-like curves across which 
$F_{i+1}$ is collapsed. The regular cell structure is obtained by restriction of cells of $X /\left(\operatorname{ker} g_{i+1}\right)$ to the resulting ball.

\section{Combinatorial 0-Hecke algebra lemmas}

The relations $x_{i} x_{i}=x_{i}$ will yield a 0 -Hecke algebra variant on the deletion exchange property for Coxeter groups, namely our upcoming notion of "deletion pair". In preparation, we first discuss a poset map from a Boolean algebra to Bruhat order.

It is natural (and will be helpful) to associate a Coxeter group element $w\left(x_{i_{1}} \cdots x_{i_{d}}\right)$ to any 0 -Hecke algebra expression $x_{i_{1}} \cdots x_{i_{d}}$. This is done by applying braid moves and modified nil-moves to $x_{i_{1}} \cdots x_{i_{d}}$ to obtain a new expression $x_{j_{1}} \cdots x_{j_{s}}$ such that $s_{j_{1}} \cdots s_{j_{s}}$ is reduced, then letting $w\left(x_{i_{1}} \cdots x_{i_{d}}\right)=s_{j_{1}} \cdots s_{j_{s}}$. The fact that this does not depend on the choice of braid moves and modified nil-moves will follow from the geometric description for $w\left(x_{i_{1}} \cdots x_{i_{d}}\right)$ given next in Proposition 5.1.

Proposition 5.1 Lusztig's map $f_{\left(i_{1}, \ldots, i_{d}\right)}$ sends $R_{S}=\left\{\left(t_{1}, \ldots, t_{d}\right) \subseteq \mathbb{R}_{\geq 0}^{d} \mid t_{i}>\right.$ 0 iff $i \in S\}$ with $S=\left\{j_{1}, \ldots, j_{k}\right\}$ to the open cell $Y_{u}^{o}$ for $u=w\left(x_{i_{j_{1}}} \cdots x_{i_{j_{k}}}\right)$.

Proof This follows from Theorem 2.18, which ensures the existence of a series of braid moves and modified nil-moves which may be applied to $x_{i_{j_{1}}} \cdots x_{i_{j_{k}}}$ mapping the points of $R_{S}$ onto the points of some cell $R_{T}$ indexed by a reduced expression, sending each $x \in R_{S}$ to some $y \in R_{T}$ with the property that $f_{\left(i_{1}, \ldots, i_{d}\right)}(x)=f_{\left(i_{1}, \ldots, i_{d}\right)}(y)$.

Corollary 5.2 The Coxeter group element $w\left(x_{i_{j_{1}}} \cdots x_{i_{j_{k}}}\right)$ does not depend on the series of braid moves and modified nil-moves used to convert $x_{i_{j_{1}}} \cdots x_{i_{j_{k}}}$ into a reduced expression.

Corollary 5.3 If $A=x_{j_{1}} \cdots x_{j_{r}}$ and $B=x_{k_{1}} \cdots x_{k_{s}}$ with $\left\{j_{1}, \ldots, j_{r}\right\} \subseteq$ $\left\{k_{1}, \ldots, k_{s}\right\}$, then $w(A) \leq$ Bruhat $w(B)$. Thus, $w$ is a poset map from a Boolean algebra to Bruhat order.

Proof $A$ is obtained from $B$ by setting some parameters to 0 , hence the open cell to which $A$ maps is in the closure of the open cell to which $B$ maps. But Bruhat order is the closure order on cells of $Y_{w}$.

See [1] for additional properties of this poset map $w$ from a Boolean algebra to Bruhat order.

The following 0-Hecke algebra notion, that of deletion pair, will play a key role in various lemmas in Sect. 6.2, i.e. in checking the combinatorial 
conditions needed in our proof of the Fomin-Shapiro Conjecture in order to use the cell collapses developed in Sect. 4.

Definition 5.4 Define a deletion pair in a 0-Hecke algebra expression $x_{i_{1}} \cdots x_{i_{d}}$ to be a pair $\left\{x_{i_{r}}, x_{i_{s}}\right\}$ such that the subexpression $x_{i_{r}} \cdots x_{i_{s}}$ is not reduced but $\hat{x}_{i_{r}} \cdots x_{i_{s}}$ and $x_{i_{r}} \cdots \hat{x}_{i_{s}}$ are each reduced.

For example, in type A the expression $x_{1} x_{2} x_{1} x_{2}$ has deletion pair $\left\{x_{i_{1}}, x_{i_{4}}\right\}$.

Lemma 5.5 If $\left\{x_{i_{r}}, x_{i_{s}}\right\}$ comprise a deletion pair, then $w\left(x_{i_{r}} \cdots x_{i_{s}}\right)=$ $w\left(\hat{x}_{i_{r}} \cdots x_{i_{s}}\right)=w\left(x_{i_{r}} \cdots \hat{x}_{i_{s}}\right)$.

Proof $w\left(x_{i_{r}} \cdots \hat{x}_{i_{s}}\right) \leq w\left(x_{i_{r}} \cdots x_{i_{s}}\right)$ and $w\left(\hat{x}_{i_{r}} \cdots x_{i_{s}}\right) \leq w\left(x_{i_{r}} \cdots x_{i_{s}}\right)$ in Bruhat order, while all three of these Coxeter group elements have the same length, so the equalities follow.

See [10] for a faithful representation of the 0-Hecke algebra in which the simple reflections which do not increase length act by doing nothing.

Remark 5.6 If $\left\{x_{i_{u}}, x_{i_{v}}\right\}$ comprise a deletion pair in a word $x_{i_{1}} \cdots x_{i_{d}}$ and we apply a braid relation in which $x_{i_{u}}$ is the farthest letter from $x_{i_{v}}$ in the segment being braided, then the resulting expression will have as a deletion pair $x_{i_{v}}$ together with the nearest letter to it in the segment that was braided. We regard this as a braided version of the same deletion pair.

Example 5.7 Applying a braid relation to $x_{1} x_{2} x_{1} x_{2}$ yields $x_{2} x_{1} x_{2} x_{2}$; we regard the third and fourth letter in the new expression as a braided version of the deletion pair comprised of the first and fourth letters in the original expression.

Given a reduced expression $x_{i_{1}} \cdots x_{i_{d}}$, associate a Coxeter group element $R\left(x_{i_{j}}\right)$ to each $x_{i_{j}}$ by letting $R\left(x_{i_{j}}\right)=s_{i_{1}} \cdots s_{i_{j-1}} s_{i_{j}} s_{i_{j-1}} \cdots s_{i_{1}}$. For finite Coxeter groups, these will be the reflections. In the case of a nonreduced expression, if $w\left(x_{i_{1}} \cdots x_{i_{j}}\right)=w\left(x_{i_{1}} \cdots x_{i_{j-1}}\right)$, then we find the largest $j^{\prime}<j$ such that $s_{i_{j^{\prime}}} \cdots s_{i_{j-1}}=s_{i_{j^{\prime}+1}} \cdots s_{i_{j}}$ and let $R\left(x_{i_{j}}\right)=R\left(x_{i_{j^{\prime}}}\right)$.

Our original proof of the next lemma relied on the fact that all finite Coxeter groups are also reflection groups. Sergey Fomin provided us with the proof presented below which avoids passing to reflection groups. In particular, this allows us to avoid assuming here, and thereby throughout the paper, that our Coxeter groups are of finite type.

Lemma 5.8 Given a reduced expression $x_{i_{1}} \cdots x_{i_{m}}$ in the 0-Hecke algebra of a Coxeter group in which $R\left(x_{i_{m}}\right)=s_{i_{0}}$, then $x_{i_{0}} x_{i_{1}} \cdots x_{i_{m}}$ has $\left\{x_{i_{0}}, x_{i_{m}}\right\}$ as a deletion pair. 
Proof Let us show that $x_{i_{0}} x_{i_{1}} \cdots x_{i_{m-1}}$ is reduced whereas $x_{i_{0}} x_{i_{1}} \cdots x_{i_{m}}$ is not. By Exercise 8 in [5, Chap. 1], it suffices to prove $R\left(x_{i_{j}}\right) \neq R\left(x_{i_{k}}\right)$ for all $0 \leq j<k \leq m-1$ along with proving $R\left(x_{i_{0}}\right)=R\left(x_{i_{m}}\right)$. But $x_{i_{1}} \cdots x_{i_{m-1}}$ is reduced, which implies $s_{i_{1}} \cdots s_{i_{j-1}} s_{i_{j}} s_{i_{j-1}} \cdots s_{i_{1}} \neq s_{i_{1}} \cdots s_{i_{k-1}} s_{i_{k}} s_{i_{k-1}} \cdots s_{i_{1}}$ for $1 \leq j<k \leq m-1$. This in turn implies $R\left(x_{i_{j}}\right) \neq R\left(x_{i_{k}}\right)$ for $1 \leq j<k \leq$ $m-1$ with respect to the expression $x_{i_{0}} x_{i_{1}} \cdots x_{i_{m}}$, since we simply conjugate the previous inequalities by $s_{i_{0}}$ to obtain the desired inequalities. On the other hand, $R\left(x_{i_{0}}\right)=s_{i_{0}}=s_{i_{0}}^{3}=s_{i_{0}}\left(s_{i_{1}} \cdots s_{i_{m}} \cdots s_{i_{1}}\right) s_{i_{0}}$, completing the proof.

\section{Proof of the Fomin-Shapiro Conjecture}

In this section, we apply Theorem 1.3 to the stratified spaces $Y_{w}$ introduced by Fomin and Shapiro in [11] to prove the following.

Theorem 6.1 The Bruhat decomposition $Y_{w}$ of the link of the identity in the totally nonnegative real part of the unipotent radical of a Borel subgroup in a semisimple, simply connected group defined and split over $\mathbb{R}$ is a regular $C W$ complex homeomorphic to a ball. Moreover the link of each cell is as well.

Much of the proof will consist of first constructing a regular CW complex $K$ that will be a quotient space $\left(\mathbb{R}_{>0}^{d} \cap S_{1}^{d-1}\right) / \sim$ of a simplex; this is obtained from the simplex by a series of collapses in Sect. 6.2. Then we prove that the quotient space map induced from $f_{\left(i_{1}, \ldots, i_{d}\right)}$ will act on $K$ in a manner that meets all the requirements of Corollary 3.9. As preparation, we first define a much simpler equivalence relation in Sect. 6.1, denoted $\sim_{C}$, doing identifications based only on stuttering pairs which may be obtained in nonreduced expressions exclusively by applying commutation moves. Then we give the more difficult analogous argument for $\sim$. Finally, Sect. 6.3 applies Theorem 1.3 to show that the induced map $\overline{f_{\left(i_{1}, \ldots, i_{d}\right)}}$ from $K$ to $Y_{w}$ is a homeomorphism.

Let us now establish some convenient notation for the proof. Let $\mathbb{R}_{\geq 0}^{d} \cap$ $S_{1}^{d-1}$ denote the restriction of $\mathbb{R}_{\geq 0}^{d}$ to the hyperplane with coordinates summing to 1 . We will make extensive use of the fact that this is a convex polytope. Define the regions or faces in $\mathbb{R}_{\geq 0}^{d} \cap S_{1}^{d-1}$ as the sets $R_{S}=\left\{\left(t_{1}, \ldots, t_{d}\right) \in\right.$ $\mathbb{R}_{\geq 0}^{d} \cap S_{1}^{d-1} \mid t_{i}>0$ iff $\left.i \in S\right\}$. We associate the 0 -Hecke algebra expression $x_{i_{j_{1}}} \cdots x_{i_{j_{k}}}$ to the region $R_{\left\{j_{1}, \ldots, j_{k}\right\}}$, calling this the $x$-expression of $R_{\left\{j_{1}, \ldots, j_{k}\right\}}$, denoted $x\left(R_{\left\{j_{1}, \ldots, j_{k}\right\}}\right)$. For subexpressions $x(A)$ and $x(B)$ of $x_{i_{1}} \cdots x_{i_{d}}$, let $x(A) \vee x(B)$ be the expression made of the union of the indexing positions from $x(A)$ and $x(B)$, and let $A \vee B$ denote the cell given by that expression. To keep track of the positions of the nonzero parameters, we sometimes also include 1's as placeholders, e.g. describing the region $R_{\{1,3\}}$ given by the map $f_{(1,2,1)}$ by the expression $x_{1} \cdot 1 \cdot x_{1}$. We say that an $x$-expression is stuttering if 
it directly admits a modified nil-move $x_{i} x_{i} \rightarrow x_{i}$, i.e. $x_{i}(u) x_{i}(v)=x_{i}(u+v)$ (cf. Sect. 2.3). An expression is commutation equivalent to a stuttering expression if it admits a series of commutation moves yielding a stuttering expression.

In Sect. 6.2, we will define the equivalence relation $\sim$ on $\mathbb{R}_{\geq 0}^{d} \cap S_{1}^{d-1}$ by repeated application of the following idea: if the $x$-expression $x_{i_{j_{1}}} \cdots x_{i_{j_{k}}}$ associated to a point $\left(t_{1}, \ldots, t_{d}\right) \in \mathbb{R}_{\geq 0}^{d}$ is not reduced, then we may apply commutation moves and long braid moves to it, causing a coordinate change to new coordinates $\left(u_{1}, \ldots, u_{d}\right)$ in which we may apply a substitution $x_{i}\left(u_{s-1}\right) x_{i}\left(u_{s}\right)=x_{i}\left(u_{s-1}+u_{s}\right)$. Each region $R_{\left\{j_{1}, \ldots, j_{k}\right\}}$ indexed by a non-reduced word is collapsed by such a move, at which point we say $\left(u_{1}, \ldots, u_{d}\right) \sim\left(u_{1}^{\prime}, \ldots, u_{d}^{\prime}\right)$ for those points $\left(u_{1}^{\prime}, \ldots, u_{d}^{\prime}\right) \in \mathbb{R}_{\geq 0}^{d} \cap S_{1}^{d-1}$ such that $u_{s-1}^{\prime}+u_{s}^{\prime}=u_{s-1}+u_{s}$ and $u_{i}^{\prime}=u_{i}$ for $i \neq s-1, s$. It is important to our collapsing argument that for each non-reduced subword $\left(i_{j_{1}}, \ldots, i_{j_{k}}\right)$ of $\left(i_{1}, \ldots, i_{d}\right)$, we choose exactly one such way of identifying points of the open cell $R_{\left\{j_{1}, \ldots, j_{k}\right\}}$ with points having strictly fewer nonzero parameters, namely the identifications dictated by the collapse we choose to apply to $R_{\left\{j_{1}, \ldots, j_{k}\right\}}$. Additional identifications will hold by transitivity of $\sim$.

Before turning to the details, let us briefly enumerate the main upcoming definitions, lemmas and theorems and how they fit together. Definition 6.6 introduces the parallel-like curves that will be used to induce the collapses leading to $\sim_{C}$. Then we verify the distinct endpoints condition (DE) in Lemma 6.9, the least upper bound condition (LUB) in Lemma 6.12, the distinct initial points condition (DIP) in Lemma 6.10, and deduce from all this the regularity of the quotient cell complex $\left(\mathbb{R}_{\geq 0}^{d} \cap S_{1}^{d-1}\right) / \sim_{C}$ in Theorem 6.13. Afterwards, we characterize exactly which faces are identified with each other by $\sim_{C}$ in Lemma 6.14 .

Now in the general case of $\sim$, we prove a similar series of lemmas, after first showing that long braid moves may be accomplished by change of coordinates maps that are homeomorphisms on the closed cells to be collapsed. The requisite parallel-like curves are specified in Definition 6.18. The result about changes of coordinates is obtained through Lemmas 6.20, 6.22, 6.23, and 6.24. Next we verify the conditions (DIP) in Lemma 6.28, (DE) in Lemma 6.29, (LUB) in Lemma 6.30, and we show the requisite equidimensionality to deduce regularity of links in Lemma 6.27. In proving these results for a particular collapse, we assume inductively that all earlier collapses were already performed successfully, and we also assume inductively that all results in the paper hold for all $d^{\prime}<d$ to prove the results for reduced word $\left(i_{1}, \ldots, i_{d}\right)$ of length $d$. We then combine these ingredients to deduce homeomorphism type and regularity of $\left(\mathbb{R}_{\geq 0}^{d} \cap S_{1}^{d-1}\right) / \sim$ in Theorem 6.32.

Finally, we prove that the induced map $\bar{f}_{\left(i_{1}, \ldots, i_{d}\right)}$ from this quotient space to $Y_{w}$ is a homeomorphism that preserves cell structure, implying that $Y_{w}$ is 
a regular CW complex homeomorphic to a ball. To this end, Lemma 6.33 uses the exchange axiom for Coxeter groups in order to verify the attaching map injectivity requirement of Theorem 1.3, allowing the proof of the FominShapiro Conjecture to be completed in Theorem 6.34.

\subsection{Collapsing a simplex to obtain $\left(\mathbb{R}_{\geq 0}^{d} \cap S_{1}^{d-1}\right) / \sim_{C}$}

In this section, we collapse those faces of $\mathbb{R}_{>0}^{d} \cap S_{1}^{d-1}$ whose words are commutation equivalent to stuttering words, denoting the resulting identifications by $\sim_{C}$. We prove that $\left(\mathbb{R}_{\geq 0}^{d} \cap S_{1}^{d-1}\right) / \sim_{C}$ is a regular CW complex homeomorphic to a ball by proving that regularity and homeomorphism type are preserved under each in a series of collapses of the type introduced in Sect. 4. A separate proof for $\sim_{C}$ is given before turning to the general case for two reasons: (1) it illustrates the general strategy in a much simpler setting, and (2) this result will be used in the proofs of Lemmas 6.20 and 6.24, two key ingredients to the general case.

Definition 6.2 An omittable pair of an $x$-expression $x(F)$ is a pair $\left\{x_{i_{l}}, x_{i_{r}}\right\}$ of letters appearing in $x(F)$ with $i_{l}=i_{r}$ such that there exists a series of commutation moves applicable to $x(F)$ placing the letters into neighboring positions, thereby enabling a modified nil-move.

Example 6.3 The $x$-expression $x_{1} x_{3} x_{4} x_{3} x_{1}$ in type A has omittable pair $\left\{x_{i_{1}}, x_{i_{5}}\right\}$, sometimes denoted simply by the positions, i.e. as $\{1,5\}$.

Order as follows all possible triples $\left(x(F),\left\{i_{l}, i_{r}\right\}\right)$, where $F$ is a face in $\mathbb{R}_{\geq 0}^{d} \cap S_{1}^{d-1}$ and $\left\{x_{i_{l}}, x_{i_{r}}\right\}$ is an omittable pair in $x(F)$ with $l<r$. Use linear order on the index $r$, then break ties with linear order on $r-l$, breaking further ties by reverse linear order on $\operatorname{dim} F$, and breaking any remaining ties arbitrarily.

Example $6.4\left(x_{1} x_{3} x_{4} x_{3} x_{1},\{1,5\}\right)$ precedes $\left(x_{1} x_{3} \cdot x_{3} x_{1},\{1,5\}\right)$ in this ordering, while $\left(x_{1} x_{3} \cdot x_{3} x_{1},\{2,4\}\right)$ comes earlier than both of these.

We obtain from this our sequence of face collapses by repeatedly choosing for the next collapse the earliest triple $\left(x(F),\left\{x_{i_{l}}, x_{i_{r}}\right\}\right)$ whose face $F$ has not yet been collapsed. Denote by $\left(x\left(F_{m}\right),\left\{x_{i_{l_{m}}}, x_{i_{r_{m}}}\right\}\right)$ the triple chosen for the $m$-th collapse, and let $g_{m}$ be the collapsing map accomplishing this, based on the level curves from Definition 6.6. Our main task in this section will be to prove that these are parallel-like curves and that $g_{m}$ meets the requirements of Theorem 4.21.

Remark 6.5 It often will happen that the step collapsing a cell $F_{m}$ will also collapse some additional cells. However, each collapsing step will have one 
cell among those being collapsed at that step such that all others being collapsed at that step are in its closure.

Definition 6.6 Given the triple $\left(x\left(F_{m}\right),\left\{x_{i_{l}}, i_{i_{r_{m}}}\right\}\right)$ specifying the $m$-th collapse, call the collections of points

$$
\left\{\left(t_{1}, \ldots, t_{d}\right) \mid t_{l_{m}}+t_{r_{m}}=k \text { and } t_{j}=k_{j} \text { for } j \notin\left\{l_{m}, r_{m}\right\}\right\}
$$

in $\bar{F}_{m}$ for the various sets of constants $\left\{k, k_{1}, \ldots, \hat{k}_{l_{m}}, \ldots \hat{k}_{r_{m}}, \ldots, k_{d}\right\} \in[0,1]$ summing to 1 the level curves of $\bar{F}_{m}$.

Notation 6.7 If cells $G$ and $G^{\prime}$ are identified during one of the first $m-1$ collapsing steps, denote this by $G \sim_{m} G^{\prime}$.

Remark 6.8 The collapse given by $\left(x\left(F_{m}\right),\left\{x_{i_{l_{m}}}, x_{i_{r_{m}}}\right\}\right)$ will also collapse those cells in $\bar{F}_{m}$ given by subexpressions of $x\left(F_{m}\right)$ having both $t_{i_{m}}>0$ and $t_{i_{r_{m}}}>0$. In this manner, the collapse will identify faces having $t_{i_{m}}=0$ with ones instead having $t_{i_{r_{m}}}=0$.

To keep track combinatorially of which faces are identified by the collapses giving rise to $\sim_{C}$, define a slide-move, or simply a slide, to be the replacement of $S=\left\{j_{1}, \ldots, j_{s}\right\}$ by $S^{\prime}=\left\{k_{1}, \ldots, k_{s}\right\}$ for $j_{1}<\cdots<j_{s}$ and $k_{1}<\cdots<k_{s}$ with $j_{i}=k_{i}$ for $i \neq r$ for some fixed $r$ and $i_{j_{r}}=i_{k_{r}}$. An example in type A for $\left(i_{1}, \ldots, i_{d}\right)=(1,2,3,1,2)$ is $S=\{1,5\}$ and $S^{\prime}=\{4,5\}$. An exchange is the replacement of one letter by another letter than can be accomplished by a series of slide-moves and commutation moves.

Now we use combinatorics to verify that the hypotheses needed for topological collapses introduced in Sect. 4 are indeed met. Condition 1 of Definition 4.7 follows immediately from our set-up. The next two lemmas check conditions 2 and 3, respectively, by checking the distinct endpoints condition (DE) and distinct initial points condition (DIP).

Lemma 6.9 The collapses inducing $\sim_{C}$ satisfy Condition $4.11(D E)$.

Proof What we must prove is that the two endpoints of any nontrivial level curve across which a cell $F_{i}$ is collapsed live in distinct cells just prior to the collapse. Suppose $G_{1} \subseteq \overline{F_{i}}$ with $t_{l_{i}}>0$ and $t_{r_{i}}=0$ had been identified already with the face $G_{2} \subseteq \overline{F_{i}}$ instead having $t_{r_{i}}>0$ and $t_{l_{i}}=0$. This would have required a series of earlier slides, including one of the form $r_{i} \rightarrow r$ for some $r<r_{i}$. Our collapsing order implies $r_{i}-r<r_{i}-l_{i}$. By our dimension maximizing assumption in our collapsing order, the last of these slide moves shifting the right element of our deletion pair to the left would have also collapsed $F_{i}$, by virtue of identifying it with a face already collapsed, a contradiction. 
Lemma 6.10 Suppose a cell $H_{1} \subseteq G_{1}$ is collapsed prior to the collapse of $F_{j}$, where $H_{1}$ is identified with $H_{2} \subseteq G_{2}$ in the collapsing step given by $\left(F_{j},\left\{x_{i_{r}}, x_{i_{s}}\right\}\right)$ by an exchange of $x_{i_{r}} \in H_{1} \subseteq G_{1}$ for $x_{i_{s}} \in H_{2} \subseteq G_{2}$ with $r<s$. Then the least upper bound $H_{1} \vee H_{2}$ will have also been collapsed prior to the collapse of $F_{j}$, and in such a way that any two level curves in $H_{1} \vee H_{2}$ having the same endpoint in $H_{1}$ are identified prior to the collapse $\left(F_{j},\left\{x_{i_{r}}, x_{i_{s}}\right\}\right)$ with coinciding parametrizations.

Proof The fact that $H_{1}$ is collapsed before $F_{j}$ means that $\left.x\left(H_{1}\right)\right|_{x_{i_{1}} \cdots x_{i_{s}}}$ contains an omittable pair. However, $\left.x\left(H_{1}\right)\right|_{x_{i_{1}} \cdots x_{i_{s}}}=\left.x\left(H_{1}\right)\right|_{x_{i_{1}} \cdots x_{i_{s-1}}}$, implying $\left.x\left(H_{1}\right)\right|_{x_{1} \cdots x_{i_{s-1}}}$ contains an omittable pair based upon which $H_{1}$ is collapsed. By our prioritization of higher dimensional faces in our collapsing order, the face $H_{1} \vee\left\{x_{i_{s}}\right\}=H_{1} \vee H_{2}$ will have been collapsed in the same way, yielding the desired result.

Next we verify (LUB), after first giving an example showing the idea.

Example 6.11 The cell $F$ with $x(F)=x_{1} x_{1} x_{1}$ is collapsed based on the deletion pair comprised of its leftmost two $x_{1}$ 's, identifying $x_{1} \cdot 1 \cdot x_{1}$ with $1 \cdot x_{1} \cdot x_{1}$ and in the process also identifying $x_{1} \cdot 1 \cdot 1$ with $1 \cdot x_{1} \cdot 1$. The region with expression $1 \cdot x_{1} \cdot x_{1}$ is collapsed based on its pair of $x_{1}$ 's, identifying $1 \cdot x_{1} \cdot 1$ with $1 \cdot 1 \cdot x_{1}$. Composing face identifications based on these two steps causes $x_{1} \cdot 1 \cdot 1$ to be identified with $1 \cdot 1 \cdot x_{1}$, potentially causing the attaching map for the face given by $x_{1} \cdot 1 \cdot x_{1}$ no longer to be injective; however, this face will itself have been collapsed by this time, by virtue of having already been identified with the face $1 \cdot x_{i} \cdot x_{i}$ which was already collapsed.

Lemma 6.12 The collapses inducing $\sim_{C}$ satisfy condition $4.19(L U B)$.

Proof Suppose that $G_{1}$ and $G_{2}$ are identified during the collapse of $F$ via deletion pair $\left\{x_{i_{l_{j}}}, x_{i_{r_{j}}}\right\}$, for $x_{i_{l_{j}}} \in x\left(G_{1}\right), x_{i_{r_{j}}} \in x\left(G_{2}\right)$, and $F^{\prime}$ is any face that is a least upper bound for $G_{1}$ and $G_{2}$ in the closure poset just prior to the collapse of $F$. We must show that $F^{\prime}$ is also collapsed by the end of the step collapsing $F$ or is identified with $F$ prior to this collapse. By virtue of our set-up, $x\left(\overline{F^{\prime}}\right)$ must have subexpressions $x\left(G_{1}^{\prime}\right)$ and $x\left(G_{2}^{\prime}\right)$ with $G_{1}^{\prime} \sim_{j} G_{1}$ and $G_{2}^{\prime} \sim_{j} G_{2}$. Consider $x\left(F^{\prime}\right)$ and its earliest subexpressions (in our collapsing ordering on triples) which are $x$-expressions for some such $G_{1}^{\prime} \sim_{j} G_{1}$ and $G_{2}^{\prime} \sim_{j} G_{2}$.

Suppose $x_{i_{r_{j}}} \notin x\left(F^{\prime}\right)$. This implies that $x_{i_{r_{j}}}$ must have been exchanged with a letter $x_{i_{r}}$ to its left during an earlier identification step. Then $x\left(F^{\prime}\right)$ will have an omittable pair $\left\{x_{i_{l}}, x_{i_{r}}\right\}$ for some $r<r_{j}$, causing $F^{\prime}$ to have been collapsed strictly before the collapse of $F$. 
Now consider the case $x_{i_{r_{j}}} \in x\left(F^{\prime}\right) . F^{\prime}$ will again be collapsed during or prior to the collapse of $F$ unless $x_{i_{r_{j}}}$ is the right letter in the leftmost deletion pair of $F^{\prime}$ and $x_{i_{l_{j}}}$ has been exchanged for a letter $x_{i_{l}} \in x\left(F^{\prime}\right)$ to its left appearing instead in $F^{\prime}$. But then by the fact that our collapsing order maximizes dimension among faces with the same omittable pair, this exchange $x_{i_{l_{j}}} \rightarrow x_{i_{l}}$ (or each such exchange in a series) would extend to a face including $x_{i_{r_{j}}}$, thereby identifying faces including $x_{i_{l}}$ and $x_{i_{r_{j}}}$ with ones instead including $x_{i_{l_{j}}}$ and $x_{i_{r_{j}}}$. In particular, this identifies $F^{\prime}$ with $F$ prior to the collapse of $F$.

Combining the above results will yield:

Theorem 6.13 $\left(\mathbb{R}_{\geq 0}^{d} \cap S_{1}^{d-1}\right) / \sim_{C}$ is a regular $C W$ complex homeomorphic to a ball.

Proof We will use Theorem 4.21 to prove that each collapse on $\mathbb{R}_{\geq 0}^{d} \cap S_{1}^{d-1}$ may be accomplished in turn by a map that preserves homeomorphism type and regularity, provided that all earlier collapses also met the requirements of Theorem 4.21. This will imply that the series of collapses producing $\left(\mathbb{R}_{\geq 0}^{d} \cap\right.$ $\left.S_{1}^{d-1}\right) / \sim_{C}$ yields a regular CW complex homeomorphic to a ball.

The parallel-like curves that we will use for the $(i+1)$-st collapsing step will be of the form given in Definition 6.6; they are the images under $g_{i} \circ \cdots \circ g_{1}$ of parallel line segments covering a closed cell of $\mathbb{R}_{\geq 0}^{d} \cap S_{1}^{d-1}$. To see that $g_{i} \circ \cdots \circ g_{1}$ acts on each level curve either homeomorphically or by sending it to a point, notice that by definition the interior of any nontrivial level curve lives entirely in some open cell $F \subseteq \overline{F_{i}}$, hence a cell upon which all earlier collapses act homeomorphically. Lemmas 6.9, 6.10, and 6.12 confirm the distinct endpoints condition (DE), distinct initial points condition (DIP), and least upper bound condition (LUB), respectively, i.e. the requirements of Theorem 6.13 .

Proposition 6.14 Suppose $x\left(R_{S}\right)$ and $x\left(R_{T}\right)$ are not commutation equivalent to stuttering expressions. Then $R_{S} \sim_{C} R_{T}$ iff $S$ and $T$ differ from each other by a series of commutation moves and slide moves.

Proof Let $S=\left\{j_{1}, \ldots, j_{S}\right\}$ and $T=\left\{k_{1}, \ldots k_{S}\right\}$. We begin with pairs of words $x\left(R_{S}\right), x\left(R_{T}\right)$ differing by a single slide, so $S \cap T=S \backslash\left\{j_{r}\right\}=T \backslash\left\{k_{r}\right\}$ for some $r$ with $i_{j_{r}}=i_{k_{r}}$. But then $x\left(R_{S \cup T}\right)$ is stuttering, implying $R_{S \cup T}$ was collapsed by $\sim_{C}$. The fact that $x\left(R_{S}\right), x\left(R_{T}\right)$ are not commutation equivalent to stuttering expressions implies $R_{S \cup T}$ could have only been collapsed by identifying $R_{S}$ with $R_{T}$. By transitivity of $\sim_{C}, S$ and $T$ differing by a 
series of slide moves likewise give rise to $R_{S}, R_{T}$ with $R_{S} \sim_{C} R_{T}$. Applying commutation moves to $x\left(R_{S}\right)$ as well to produce $\sigma\left(x\left(R_{\sigma(S)}\right)\right)$ which is slide equivalent to $x\left(R_{T}\right)$ ensures $x\left(R_{S \cup T}\right)$ also admits the same commutation moves leading to a stuttering word, and again $x\left(R_{S \cup T}\right)$ does not admit any other stuttering pairs, so again $R_{S} \sim_{C} R_{T}$.

Example 6.15 (a) For $\left(i_{1}, \ldots, i_{d}\right)=(1,2,1)$ in type $\mathrm{A}, R_{\{1\}}$ is identified with $R_{\{3\}}$ during the collapse of $R_{\{1,3\}}$. (b) If $\left(i_{1}, \ldots, i_{d}\right)=(1,3,1)$, then $R_{\{1,2\}}$ is identified with $R_{\{2,3\}}$ during the collapse of $R_{\{1,2,3\}}$.

\subsection{Collapsing a simplex to obtain $\left(\mathbb{R}_{\geq 0}^{d} \cap S_{1}^{d-1}\right) / \sim$}

Now we turn to the identifications $\sim$ induced by a series of collapses which collapse all faces whose words are nonreduced, i.e. starting afresh so as now to incorporate those collapses requiring long braid moves.

Definition 6.16 Given a deletion pair $\left\{x_{i_{r}}, x_{i_{s}}\right\}$ with $r<s$ in $x(F)$, let $c\left(\left\{x_{i_{r}}, x_{i_{s}}\right\} ; x(F)\right)$ be the smallest number of long braid moves needed in a series of braid moves applied to $x_{i_{r}} \cdots x_{i_{s-1}}$ yielding an expression whose last letter comprises a stutter with $x_{i_{s}}$.

Lemma 5.5 combined with Theorem 2.18 guarantees existence and finiteness of $c\left(\left\{x_{i_{r}}, x_{i_{s}}\right\} ; x(F)\right)$.

Example 6.17 In type $\mathrm{A}$, we have $c\left(\left\{x_{i_{1}}, x_{i_{4}}\right\} ; x_{1} x_{2} x_{1} x_{2}\right)=1$, because we may apply the relation $x_{1} x_{2} x_{1} \rightarrow x_{2} x_{1} x_{2}$ to obtain $x_{2} x_{1} x_{2} x_{2}$.

Now let us order triples $\left(x(F),\left\{x_{i_{l}}, x_{i_{r}}\right\}\right)$ where $\left\{x_{i_{l}}, x_{i_{r}}\right\}$ is a deletion pair of $x(F)$ in preparation for our choice of a collapsing order on nonreduced faces. By convention, say $l<r$. Letting the statistics listed earliest take highest priority, with later statistics used to break ties, order the triples ( $\left.x(F),\left\{x_{i_{l}}, x_{i_{r}}\right\}\right)$ by: (1) linear order on $r$, (2) linear order on $r-l$, (3) linear order on $c\left(\left\{x_{i_{1}}, x_{i_{r}}\right\} ; x(F)\right)$, and (4) reverse linear order on $\operatorname{dim} F$. We may break any remaining ties arbitrarily.

We repeatedly choose the earliest triple $\left(x(F),\left\{x_{i_{l}}, x_{i_{r}}\right\}\right)$ among those for faces $F$ not yet collapsed. Denote the $k$-th such triple chosen by $\left(x\left(F_{k}\right),\left\{x_{i_{l_{k}}}, x_{i_{r_{k}}}\right\}\right)$. We will use Theorem 4.21 to accomplish the collapse of $F_{k}$ by a collapsing map $g_{k}$ applied to $\left(\mathbb{R}_{\geq 0}^{d} \cap S_{1}^{d-1}\right) / \sim_{k}$, letting $\sim_{k}$ be the equivalence relation comprised of the identifications that result from the first $k-1$ collapsing steps, described shortly. First we will need some results regarding change of coordinate maps.

Denote by $\sim^{s}$ the set of all possible identifications $\left(t_{1}, \ldots, t_{d}\right) \sim\left(t_{1}^{\prime}\right.$, $\left.\ldots, t_{d}^{\prime}\right)$ under $\sim$ where $f_{\left(i_{1}, \ldots, i_{s}\right)}\left(t_{1}, \ldots, t_{s}\right)=f_{\left(i_{1}, \ldots, i_{s}\right)}\left(t_{1}^{\prime}, \ldots, t_{s}^{\prime}\right)$ and $t_{j}=t_{j}^{\prime}$ 
for all $j>s$. That is, $\sim^{s}$ consists of all possible identifications based on the leftmost $s$ letters. By our inductive hypothesis based on length, we will be able to assume $\sim^{s}$ is exactly the identifications accomplished by collapses based on deletion pairs involving only the leftmost $s$ letters. The way our collapsing order was chosen implies then that for each $s$ there will be some $k$ such that $\sim^{s}=\sim_{k}$, meaning that $\sim^{s}$ is the equivalence relation resulting from the first $k-1$ collapses. For this same pair $k$ and $s$, let us also introduce the notation $g^{s}$ for the composition of maps $g^{s}=g_{k-1} \circ \cdots \circ g_{1}$. Let us also establish the notation $g_{\left(i_{1}, \ldots, i_{d}\right)}^{s}$ as the map $g^{s}$ given by the reduced word $\left(i_{1}, \ldots, i_{d}\right)$. Let $\sim_{\left(i_{1}, \ldots, i_{d}\right)}$ denote the equivalence relation given by reduced word $\left(i_{1}, \ldots, i_{d}\right)$ after all possible collapses, and let $\sim_{\left(i_{1}, \ldots, i_{d}\right)}^{s}$ denote the equivalence relation $\sim^{s}$ consisting of identifications based on the leftmost $s$ letters again with respect to initial choice of reduced word $\left(i_{1}, \ldots, i_{d}\right)$.

Definition 6.18 Given the triple $\left(x\left(F_{k}\right),\left\{x_{i_{l_{k}}}, x_{i_{r_{k}}}\right\}\right)$, choose a sequence of braid moves on $\left(i_{l_{k}}, \ldots, i_{r_{k}-1}\right)$ using exactly $c\left(x(F),\left\{x_{i_{l_{k}}}, x_{i_{r_{k}}}\right\}\right)$ long braid moves to transform $\left(i_{1}, \ldots, i_{d}\right)$ into $\left(j_{1}, \ldots, j_{d}\right)$ with a stutter $j_{r_{k}-1}=j_{r_{k}}$. Obtain new coordinates $\left(u_{1}, \ldots, u_{d}\right)$ on $\overline{F_{k}} / \sim_{k}$ as the unique solution (up to equivalence relation $\sim_{k}$ ) to

$$
f_{\left(i_{1}, \ldots, i_{d}\right)}\left(t_{1}, \ldots, t_{d}\right)=f_{\left(j_{1}, \ldots, j_{d}\right)}\left(u_{1}, \ldots, u_{d}\right)
$$

which has $u_{i}=t_{i}$ for $i \notin\left\{l_{k}, \ldots, r_{k}-1\right\}$, as justified by Lemmas 6.20 and 6.24. The level curves for this triple are the collections of points

$$
\left\{\left(u_{1}, \ldots, u_{d}\right) \mid u_{r_{k}-1}+u_{r_{k}}=c \text { and } u_{m}=c_{m} \text { for all } m \notin\left\{r_{k}-1, r_{k}\right\}\right\}
$$

for the various choices of constants $c, c_{1}, \ldots, \hat{c}_{r_{k}-1}, \hat{c}_{r_{k}}, \ldots, c_{d} \geq 0$.

We will eventually prove that these level-curves are parallel-like, in the sense of Definition 4.7.

Example 6.19 Applying braid moves to $x_{1} x_{2} x_{1} x_{3} x_{2} x_{3}$ yields the expression $x_{2} x_{1} x_{3} x_{2}\left(x_{3} x_{3}\right)$. Collapsing based on the resulting stuttering pair will cause the proper face $1 \cdot x_{2} x_{1} x_{3} x_{2} x_{3}$ to be identified with the face $x_{1} x_{2} x_{1} x_{3} x_{2} \cdot 1$. The proper face $x_{1} x_{2} \cdot 1 \cdot 1 \cdot 1 \cdot x_{3}$ is neither collapsed nor identified with another face in the process since the first and last letters do not form a deletion pair. On th e other hand, the face $x_{1} x_{2} \cdot 1 \cdot x_{3} x_{2} x_{3}$ would have already been collapsed at an earlier step, hence need not be considered in the next lemma as part of the boundary of the cell indexed by $x_{1} x_{2} x_{1} x_{3} x_{2} x_{3}$.

Lemma 6.20 Consider the reduced expression $s_{i} s_{j} \ldots$ of length $m(i, j)$ comprised of alternating $s_{i}$ 's and $s_{j}$ 's. Then the resulting regular $C W$ complex $\Delta=\left(\mathbb{R}_{\geq 0}^{m(i, j)} \cap S_{1}^{m(i, j)-1}\right) / \sim_{C}$ given by $(i, j, \ldots)$ is homeomorphic 
via the map $f_{(j, i, \ldots)}^{-1} \circ f_{(i, j, \ldots)}$ to the regular $C W$ complex $\Delta^{\prime}=\left(\mathbb{R}_{\geq 0}^{m(i, j)} \cap\right.$ $\left.S_{1}^{m(i, j)-1}\right) / \sim_{C^{\prime}}$ given by $(j, i, \ldots)$.

Proof We may use the fact that $f_{(i, j, \ldots)}$ and $f_{(j, i, \ldots)}$ act homeomorphically on the interior of the big cell for $\Delta$ and $\Delta^{\prime}$, respectively. Each point $x \in \Delta$ not in the interior of the big cell must instead belong to a region $R_{\left\{i_{j_{1}}, \ldots, i_{j_{k}}\right\}}$ whose associated Coxeter group element $w\left(x_{i_{1}} \cdots x_{i_{j_{k}}}\right)$ has a unique reduced expression, namely one with the appropriate alternation of $s_{i}$ 's and $s_{j}$ 's. Thus, $x$ must be sent to a point in $\Delta^{\prime}$ having this same reduced expression, so that by Proposition 6.14 the only choices to be made are equivalent to each other under $\sim_{C^{\prime}}$. This map from $\Delta$ to $\Delta^{\prime}$ is therefore a composition of two homeomorphisms, namely $f_{(i, j, \ldots)}$ and $f_{(j, i, \ldots)}^{-1}$, and hence is itself a homeomorphism.

Example 6.21 The type A relation $s_{i} s_{i+1} s_{i}=s_{i+1} s_{i} s_{i+1}$ gives rise to the map $\left(t_{1}, t_{2}, t_{3}\right) \rightarrow\left(t_{1}^{\prime}, t_{2}^{\prime}, t_{3}^{\prime}\right)$ for $\left(t_{1}^{\prime}, t_{2}^{\prime}, t_{3}^{\prime}\right)=\left(\frac{t_{2} t_{3}}{t_{1}+t_{3}}, t_{1}+t_{3}, \frac{t_{1} t_{2}}{t_{1}+t_{3}}\right)$ on the interior of $\left\{f_{(1,2,1)}\left(t_{1}, t_{2}, t_{3}\right) \mid t_{1}, t_{2}, t_{3} \geq 0\right\}$. The above proposition shows that this map extends to the boundary, i.e. extends to a map from $\left(\mathbb{R}_{\geq 0}^{d} \cap S_{1}^{d-1}\right) / \sim_{C}$ to $\left(\mathbb{R}_{\geq 0}^{d} \cap S_{1}^{d-1}\right) / \sim_{C^{\prime}}$. For instance, it sends $\left(t_{1}, t_{2}, 0\right)$ to $\left(0, t_{1}, t_{2}\right)$ for $t_{1}, t_{2}>0$, and it sends $\left(0, t_{2}, 0\right)$ to the $\sim_{C^{\prime}}$-equivalence class $\left\{\left(t_{1}^{\prime}, 0, t_{3}^{\prime}\right) \mid t_{1}^{\prime}+t_{3}^{\prime}=t_{2}\right\}$.

Lemma 6.22 Given a reduced word $\left(i_{1}, \ldots, i_{d}\right)$ and some $d^{\prime}<d$ such that the series of collapses for $\left(i_{1}, \ldots, i_{d^{\prime}}\right)$ successfully applies to $\mathbb{R}_{\geq 0}^{d^{\prime}} \cap S_{1}^{d^{\prime}-1}$, with each collapse preserving regularity and homeomorphism type, then the extension to $\mathbb{R}_{\geq 0}^{d} \cap S_{1}^{d-1}$ of this same series of collapses may be successfully performed on $\mathbb{R}_{\geq 0}^{d} \cap S_{1}^{d-1}$.

Proof First apply each collapse to the subcomplex of $\mathbb{R}_{\geq 0}^{d} \cap S_{1}^{d-1}$ in which $t_{d^{\prime}+1}=\cdots=t_{d}=0$, since this is exactly $\mathbb{R}_{\geq 0}^{d^{\prime}} \cap S_{1}^{d^{\prime}-1}$. Then extend continuously to the family of subspaces with $t_{d^{\prime}+1}=\cdots=t_{d}=k$ for $0 \leq k \leq \frac{1}{d-d^{\prime}+1}$, using that each of these for $k<\frac{1}{d-d^{\prime}+1}$ is also isomorphic to $\mathbb{R}_{\geq 0}^{d^{\prime}} \cap S_{1}^{d^{\prime}-1}$ and that the slice given by $k=\frac{1}{d-d^{\prime}+1}$ is a 1-point space. Geometrically, we are adding a cone point and extending the collapse to the coned complex. Continuity follows from the fact that the level curves we collapse across each hold the values $t_{d^{\prime}+1}, \ldots, t_{d}$ constant, hence the collapses apply to all (nontrivial) cross-sectional slices in the same way.

Lemma 6.23 Suppose that the $k$-th collapsing step uses deletion pair $\left\{x_{i_{l_{k}}}, x_{i_{r_{k}}}\right\}$ for $l_{k}<r_{k}$. Then $\sim^{r_{k}-1}=\sim_{m}$ where $m$ is the largest possible positive integer such that the triple $\left(x\left(F_{m}\right),\left\{x_{i_{l_{m}}}, x_{i_{r_{m}}}\right\}\right)$ has $r_{m}<r_{k}$. 
Proof Consider the map $f_{\left(i_{1}, \ldots, i_{r_{k}-1}\right)}$ on just the leftmost $r_{k}-1$ positions in our reduced word. By induction on wordlength, specifically our assumption of all results in the paper for all $d^{\prime}<d$, we may use Theorem 6.34 to deduce that $\overline{f_{\left(i_{1}, \ldots, i_{r_{k}-1}\right)}}$ is a homeomorphism from $\left(\mathbb{R}_{\geq 0}^{r_{k}-1} \cap S_{1}^{r_{k}-2}\right) / \sim$ to $Y_{s_{i_{1}} \cdots s_{i_{r_{k}-1}}}$. By Lemma 6.22, this means in particular that the collapses based on deletion pairs using only positions $1, \ldots, r_{k}-1$ are enough to accomplish all the desired identifications in $\sim_{k}^{r_{k}-1}$.

Lemma 6.24 For each $s<d$, there is a cell structure preserving homeomorphism ch from $\left(\mathbb{R}_{\geq 0}^{d} \cap S_{1}^{d-1}\right) / \sim_{\left(i_{1}, \ldots, i_{d}\right)}^{s}$ to $\left(\mathbb{R}_{\geq 0}^{d} \cap S_{1}^{d-1}\right) / \sim_{\left(j_{1}, \ldots, j_{d}\right)}^{s}$ where $\left(j_{1}, \ldots, j_{d}\right)$ is obtained from $\left(i_{1}, \ldots, i_{d}\right)$ by braid moves involving only the leftmost $s$ letters in $\left(i_{1}, \ldots, i_{d}\right)$.

Proof The case of short braid moves is obvious, since we just switch the order of the parameters. Now by induction on $d$, we may assume the main results of the paper for all $s<d$ in our proof of all these results for our given $d$, provided we check the base case of the induction. In particular, this inductive usage of Theorem 6.34 for $s<d$ together with Lemma 6.22 and the present lemma for $s<d$ gives that the two complexes under consideration, namely $\left(\mathbb{R}_{\geq 0}^{d} \cap S_{1}^{d-1}\right) / \sim_{\left(i_{1}, \ldots, i_{d}\right)}^{s}$ and $\left(\mathbb{R}_{\geq 0}^{d} \cap S_{1}^{d-1}\right) / \sim_{\left(j_{1}, \ldots, j_{d}\right)}^{s}$, both are homeomorphic by cell preserving homeomorphisms to the join $Y_{u} * \Delta_{d-s-1}$ where $\left(i_{1}, \ldots, i_{s}\right)$ and $\left(j_{1}, \ldots, j_{s}\right)$ are both reduced words for Coxeter group element $u$ and $\Delta_{d-s-1}$ is a simplex having $d-s$ vertices. Thus, by composing homeomorphisms they are also homeomorphic to each other. The base case of this induction follows immediately from Lemma 6.20.

Remark 6.25 For any braid relation $\left(s_{i} s_{j}\right)^{m(i, j)}=e$ in $W$ and any positive reals $t_{2}, \ldots, t_{m(i, j)}>0$, there is a unique $\left(t_{1}^{\prime}, \ldots, t_{m(i, j)}^{\prime}\right) \in \mathbb{R}_{\geq 0}^{m(i, j)}$ satisfying

$$
x_{i}(0) x_{j}\left(t_{2}\right) x_{i}\left(t_{3}\right) \cdots=x_{j}\left(t_{1}^{\prime}\right) x_{i}\left(t_{2}^{\prime}\right) x_{j}\left(t_{3}^{\prime}\right) \cdots,
$$

namely $t_{1}^{\prime}=t_{2}, t_{2}^{\prime}=t_{3}, \ldots, t_{m(i, j)-1}^{\prime}=t_{m(i, j)}$, and $t_{m(i, j)}^{\prime}=0$.

Remark 6.26 The proof of Lemma 6.24 immediately implies for each face $F$ with associated word $\left(i_{1}^{\prime}, \ldots, i_{d^{\prime}}^{\prime}\right)$ that is a subword of $\left(i_{1}, \ldots, i_{d}\right)$ and for any word $\left(j_{1}^{\prime}, \ldots, j_{d^{\prime}}^{\prime}\right)=\operatorname{ch}\left(i_{1}^{\prime}, \ldots, i_{d^{\prime}}^{\prime}\right)$ obtained by braiding the leftmost $s$ letters in $\left(i_{1}^{\prime}, \ldots, i_{d^{\prime}}^{\prime}\right)$ for $s \leq \min \left(d-1, d^{\prime}\right)$ using the same braid moves as above that $g_{\left(i_{1}^{\prime}, \ldots, i_{d^{\prime}}^{\prime}\right)}^{s}\left(\bar{F}_{k}\right)$ is homeomorphic to $\left.\left(\mathbb{R}_{\geq 0}^{d^{\prime}} \cap S_{1}^{d^{\prime}-1}\right) / \sim_{\left(j_{1}^{\prime}, \ldots, j_{d^{\prime}}^{\prime}\right.}^{s}\right)$.

Next we check a condition that will be helpful for verifying our various requirements for performing collapses, i.e. for checking the hypotheses of 
Corollary 4.24, assuming that all earlier collapses were performed successfully. Note that in the Lemma 6.27 we do not require $F$ to be the maximal face that is collapsed in this step.

Lemma 6.27 If a cell $F$ is collapsed across level curves each having one endpoint in $H_{1}$ and the other endpoint in $H_{2}$, then $\operatorname{dim}\left(H_{1}\right)=\operatorname{dim}\left(H_{2}\right)=$ $\operatorname{dim}(F)-1$, with neither $H_{1}$ nor $H_{2}$ collapsed earlier.

Proof Let $\left\{x_{i_{r}}, x_{i_{s}}\right\}$ be the deletion pair inducing the collapse of $F$, with $x_{i_{r}} \in x\left(H_{1}\right)$ and $x_{i_{s}} \in x\left(H_{2}\right)$. Then $\left.x\left(H_{1}\right)\right|_{x_{1} \cdots x_{i s}}$ must be reduced, since otherwise $H_{1}$ and likewise $F$ would have been collapsed earlier. This implies $\left.x\left(H_{2}\right)\right|_{x_{i_{1}} \cdots x_{i_{s}}}$ must also be reduced. Thus, neither $H_{1}$ nor $H_{2}$ will have been collapsed earlier, from which the result follows by comparing wordlengths.

Now we check the various hypotheses of Theorem 4.21 (as extended in Corollary 4.24), using our collapses and parallel-like curves from Definition 6.18 and from the discussion just after Example 6.17 as applied to our framework. To check these conditions for the $k$-th collapse, we assume by induction that all earlier collapses were performed successfully. In particular, this means that we assume that we had a regular CW complex after each earlier collapsing step and hence that (LUB) held after each earlier collapsing step. However, we do not need to check (DIP) or (DE) for the curves used in the $k$-th collapse at each earlier step. Rather, it suffices to check these conditions just before the $k$-th collapse for the curves used to accomplish the $k$-th collapse.

The fact that the level curves to be used in our collapses, defined in Definition 6.18, satisfy the first requirement for parallel-like curves is immediate from our set-up. Now let us confirm that the level curves used for the $k$-th collapse also satisfy the third requirement of parallel-like curves, the (DIP) condition, just prior to the $k$-th collapsing step, using that earlier collapses were all performed successfully.

When a collapse identifies cells $A$ and $A^{\prime}$ via a deletion pair $\left\{x_{i_{u}}, x_{i_{v}}\right\}$, we say that $x_{i_{u}}$ is exchanged for $x_{i_{v}}$, denoted $x_{i_{u}} \rightarrow x_{i_{v}}$.

Lemma 6.28 If a cell $H_{1} \subseteq G_{1}$ is collapsed prior to the collapse of $F_{j}$, where $H_{1}$ is to be identified with $H_{2}$ in the collapsing step given by $\left(F_{j},\left\{x_{i_{r}}, x_{i_{s}}\right\}\right)$ by an exchange of $x_{i_{r}} \in H_{1}$ for $x_{i_{s}} \in H_{2}$ for $r<s$, then $H_{1} \vee H_{2}$ is also collapsed prior to $F_{j}$, and in such a way that any two level curves with the same endpoint in $H_{1}$ will have already been identified with each other in a manner that preserves the parametrization.

Proof Given the two $x$-expressions $\left.x\left(H_{2}\right)\right|_{x_{i_{1}} \cdots x_{i_{s}}}=x_{i_{j_{1}}} \cdots \hat{x}_{i_{r}} \cdots x_{i_{s}}$ and $\left.x\left(H_{1}\right)\right|_{x_{1} \cdots x_{i_{s}}}=x_{i_{j_{1}}} \cdots x_{i_{r}} \cdots \hat{x}_{i_{s}}$, the fact that $H_{1}$ has already been collapsed 
means $x_{i_{j_{1}}} \cdots x_{i_{r}} \cdots \hat{x}_{i_{s}}$ is not reduced, implying that $x_{i_{j_{1}}} \cdots x_{i_{r}} \cdots x_{i_{s}}=$ $x\left(H_{1} \vee H_{2}\right)$ also is not reduced. Moreover, $H_{1}$ will have been collapsed based on a deletion pair strictly to the left of $x_{i_{s}}$, which implies the same for $H_{1} \vee H_{2}$. Our collapsing order ensures that $H_{1} \vee H_{2}$ will also have been collapsed prior to the collapse of $F_{j}$, using the same deletion pair and the same series of braid moves as in $H_{1}$, hence the same parametrization for each curve in $H_{1}$ as in the curves with which it is identified in $H_{1} \vee H_{2}$.

Next we verify that the second requirement for parallel-like curves, Condition (DE), holds just prior to the $k$-th collapse for the curves used to accomplish the $k$-th collapse.

Lemma 6.29 Condition 4.11 (DE) holds at the $k$-th collapsing step, provided that the earlier collapses were performed successfully.

Proof Suppose $F$ is collapsed during the $k$-th collapsing step via deletion pair $\left\{x_{l_{k}}, x_{r_{k}}\right\}$ having parameters $\left\{t_{l_{k}}, t_{r_{k}}\right\}$. We are not assuming $F$ is necessarily the maximal face $F_{k}$ among those faces which are collapsed at this step. Let $\left\{u_{r_{k}-1}, u_{r_{k}}\right\}$ be the new parameters for the stuttering pair obtained from the deletion pair $\left\{x_{l_{k}}, x_{r_{k}}\right\}$ by a suitable coordinate change as in Lemma 6.24. Let $G_{1}$ and $G_{2}$ be the closed faces containing opposite endpoints of the curves across which $F$ is collapsed, i.e. the curves introduced in Definition 6.18. Thus, $G_{1}$ has $t_{r_{k}}=0$ and $G_{2}$ has $t_{l_{k}}=0$, with $x\left(G_{1}\right), x\left(G_{2}\right)$ and $x(F)$ agreeing at all other positions. In the new coordinates, $G_{1}$ has $u_{r_{k}}=0$ while $G_{2}$ instead has $u_{r_{k}-1}=0$.

What we must prove is that $G_{1}$ and $G_{2}$ are not identified in an earlier collapse. Suppose otherwise. By induction, we may assume that the complex is regular immediately after each earlier collapsing step. This precludes $G_{1}$ and $G_{2}$ from being incomparable in the closure poset just prior to their identification, unless there is a face $G$ having $G_{1}, G_{2} \subseteq \bar{G}$ which is also identified with both of them at that same earlier step by collapsing $G$ across parallellike curves each having one endpoint in $G_{1}$ and the other endpoint in $G_{2}$. But then we may use (LUB) for that earlier collapse to deduce that all least upper bounds for $G_{1}$ and $G_{2}$ must have been collapsed at this earlier step, which would necessarily include a face in $\bar{F}$ other than $F$ itself. But this implies that $G_{1}$ and $G_{2}$ both have dimension at least two less than $F$, contradicting what we just proved in Lemma 6.27. Suppose on the other hand we have $G_{1} \subseteq \overline{G_{2}}$ or $G_{2} \subseteq \overline{G_{1}}$ just prior to their identification. But this implies $\operatorname{dim}\left(G_{1}\right) \neq \operatorname{dim}\left(G_{2}\right)$, again contradicting our result from Lemma 6.27 that $\operatorname{dim}\left(G_{1}\right)=\operatorname{dim}\left(G_{2}\right)=\operatorname{dim}(F)-1$.

In the proof of the next lemma, we often speak of a cell $A \in\left(\mathbb{R}_{\geq 0}^{d} \cap\right.$ $\left.S_{1}^{d-1}\right) / \sim_{k}$, whereby we mean the equivalence class under $\sim_{k}$ that contains 
the cell $A$ from $\mathbb{R}_{\geq 0}^{d} \cap S_{1}^{d-1}$. Referring in this manner to particular equivalence class representatives has the advantage that there is a unique associated $x$-expression $x(A)$ for that representative and also allows us to make sense of $A \vee B$ as being the equivalence class under $\sim_{k}$ that includes the join of the representatives $A$ and $B$ where join is taken in the original simplex $\mathbb{R}_{\geq 0}^{d} \cap S_{1}^{d-1}$. Thus, $x(A) \vee x(B)=x(A \vee B)$ is the $x$-expression comprised of the union of the $x$-expressions for $A$ and $B$. In particular, $x\left(F_{k}\right)$ denotes the $x$-expression for the $\sim_{k}$-equivalence class representative with respect to which $F_{k}$ is collapsed, namely one yielding optimal $x$-expression in terms of our collapsing order.

Lemma 6.30 Condition 4.19 (LUB) holds at the $k$-th collapsing step, provided that all earlier collapsing steps were performed successfully.

Proof Suppose that the collapsing step given by a triple $\left(F_{k},\left\{x_{i_{l_{k}}}, x_{i_{r_{k}}}\right\}\right)$ causes a pair of cells $A$ and $B$ to be identified where neither cell has been collapsed yet and neither is in the closure of the other (the latter of which would make the result trivial). By virtue of our collapsing process, this implies that we may choose $x(A)$ and $x(B)$ to be subexpressions of $x\left(F_{k}\right)$ which coincide except in that $x(A)$ includes the letter $x_{i_{r_{k}}}$ whereas $x(B)$ instead includes the letter $x_{i_{l_{k}}}$. In other words, there must exist $\sim_{k}$ equivalence class representatives with this property. Let $F$ be the cell such that $x(F)=x(B) \vee\left\{x_{i_{r_{k}}}\right\}=x(A) \vee\left\{x_{i_{l_{k}}}\right\}$. We note that $F$ might equal $F_{k}$, or $F$ might be a lower-dimensional cell contained in the closure $\bar{F}_{k}$. By definition, $F$ has dimension exactly one more than $A$ and $B$. Our collapsing order ensures that $F$ could not have been collapsed prior to our current step collapsing $F_{k}$ unless it were done through the earlier identification of $F$ with another cell $F^{\prime}$ designated for collapse earlier than $F$, where $F^{\prime}$ is obtained from $F$ by replacing $x_{i_{r_{k}}}$ with some $x_{i_{l^{\prime}}}$ with $l_{k}<l^{\prime}<r_{k}$; however, such a step would have also identified $A$ with $B$ earlier, a contradiction. Thus, we are assured of the existence of such an $F$ which is not collapsed prior to step $k$ and which satisfies $x(F)=x(A) \vee x(B)$ and $\operatorname{dim}(F)=\operatorname{dim}(A)+1=\operatorname{dim}(B)+1$.

By virtue of our collapsing process, the collapse of $F_{k}$ will induce at the same time the collapse of $F$ across curves as defined in Definition 6.18 (which we will eventually prove are parallel-like) with each curve having one endpoint in $A$ and the other endpoint in $B$. Lemma 6.23 allows us to assume that all possible identifications present in $\sim^{r_{k}-1}$ have already been accomplished before the $k$-th collapse. Let $x\left(F^{\prime}\right)$ be an $x$-expression for any other cell besides $F$ that is a least upper bound for $A$ and $B$ just prior to the $k$-th collapse. If there is such an $F^{\prime}$ distinct from $F$, then there must be cells $A_{u}, B_{v} \subseteq \bar{F}^{\prime}$ having $x\left(A_{u}\right)$ and $x\left(B_{v}\right)$ as subexpressions of $x\left(F^{\prime}\right)$ with $x\left(F^{\prime}\right)=x\left(A_{u}\right) \vee x\left(B_{v}\right), A \sim_{k} A_{u}$ and $B \sim_{k} B_{v}$. Our plan now is to obtain 
from this a contradiction so as to deduce that no such $F^{\prime}$ exists, thereby proving (LUB). To this end, we will prove either (1) that $F^{\prime}$ was already identified with $F$ at an earlier step, (2) that $F^{\prime}$ is not a least upper bound for $A$ and $B$ just prior to the $k$-th collapse due to the presence of an upper bound strictly contained in it, or (3) that $A_{u} \sim_{k} B_{v}$.

The fact that we have only done identifications based on deletion pairs whose right endpoint is at or to the left of $x_{i_{r_{k}}}$ prior to the $k$-th collapsing step implies that $A, A_{u}, B$, and $B_{v}$ all must coincide with each other to the right of $x_{i_{r_{k}}}$. We also know that $w\left(\left.A_{u}\right|_{x_{i_{1}} \cdots x_{i_{r_{k}}}}\right)=w\left(\left.A\right|_{x_{i_{1}} \cdots x_{i_{k}}}\right)$ and $w\left(\left.B_{v}\right|_{x_{i_{1}} \cdots x_{i_{r_{k}}}}\right)=w\left(\left.B\right|_{x_{i_{1}} \cdots x_{i_{k}}}\right)$, since $A \sim_{k} A_{u}$ and $B \sim_{k} B_{v}$. By definition of deletion pair, we have $w\left(\left.A\right|_{x_{i_{1}} \cdots x_{i_{r_{k}}}}\right)=w\left(\left.B\right|_{x_{i_{1}} \cdots x_{i_{r_{k}}}}\right)$, which implies $w\left(\left.A_{u}\right|_{x_{i_{1}} \cdots x_{i_{k}}}\right)=w\left(\left.B_{v}\right|_{x_{i_{1}} \cdots x_{i_{k}}}\right)$ as well.

Now consider the case where $x_{i_{r_{k}}} \notin x\left(F^{\prime}\right)=x\left(A_{u}\right) \vee x\left(B_{v}\right)$. It follows immediately from $w\left(\left.x(A)\right|_{x_{i_{1}} \cdots x_{r_{r_{k}}}}\right)=w\left(x(B)_{x_{i_{1}} \cdots x_{r_{r_{k}}}}\right)$ that we also have $w\left(\left.A_{u}\right|_{x_{i_{1}} \cdots x_{r_{r_{k}-1}}}\right)=w\left(\left.B_{v}\right|_{x_{i_{1}} \cdots x_{r_{r_{k}-1}}}\right)$. But now we may use Lemma 6.23 to conclude that $A_{u}$ must get identified with $B_{v}$ under one of the steps leading to $\sim^{r_{k}-1}$ and hence prior to step $k$. But by Lemma 6.22 , this implies $A_{u} \sim_{k} B_{v}$, completing this case.

The remainder of the proof deals with the case $x_{i_{r_{k}}} \in x\left(F^{\prime}\right)=x\left(A_{u} \vee\right.$ $\left.B_{v}\right)=x\left(A_{u}\right) \vee x\left(B_{v}\right)$. First suppose that both $x\left(A_{u}\right)$ and $x\left(B_{v}\right)$ include the letter $x_{i_{r_{k}}}$. Notice that $\left.x\left(A_{u}\right)\right|_{x_{i_{1}} \cdots x_{i_{r_{k}}-1}}$ and $\left.x\left(B_{v}\right)\right|_{x_{i_{1}} \cdots x_{r_{r_{k}-1}}}$ must both be reduced, since neither of these cells has been collapsed yet. We also have $w\left(\left.A_{u}\right|_{x_{i_{1}} \cdots x_{i_{r_{k}}}}\right)=w\left(\left.B_{v}\right|_{x_{i_{1}} \cdots x_{i_{r_{k}}}}\right)$. Hence, if $x_{i_{r_{k}}}$ is nonredundant in both $A_{u}$ and $B_{v}$, then this together implies $w\left(\left.A_{u}\right|_{x_{i_{1}} \cdots x_{r_{r_{k}-1}}}\right)=w\left(\left.B_{v}\right|_{x_{i_{1}} \cdots x_{i_{r_{k}-1}}}\right)$. These two words $x\left(A_{u}\right)$ and $x\left(B_{v}\right)$ also coincide on the subexpression consisting of $x_{i_{r_{k}}}$ and all letters to its right, implying $A_{u} \sim^{r_{k}-1} B_{v}$ and hence $A_{u} \sim_{k} B_{v}$, a contradiction. Next suppose that $x_{i_{r_{k}}}$ is present but redundant in $x\left(B_{v}\right)$, that is, suppose $w\left(\left.x\left(B_{v}\right)\right|_{x_{i_{1}} \cdots x_{i_{r_{k}-1}}}\right)=w\left(\left.x\left(B_{v}\right)\right|_{x_{i_{1}} \cdots x_{i_{r_{k}}}}\right)$; also suppose $x_{i_{r_{k}}}$ is present in $x\left(A_{u}\right)$. Then consider $x\left(B_{v}^{\prime}\right)$ obtained from $x\left(B_{v}\right)$ by deleting $x_{i_{r_{k}}}$. Then $A_{u} \vee B_{v}=A_{u} \vee B_{v}^{\prime}$, which means that it suffices to show that $A_{u} \vee B_{v}^{\prime}$ is collapsed by the end of the $k$-th collapsing step so as to deduce that $A_{u} \vee B_{v}^{\prime}$ is also collapsed by the end of the $k$-th collapsing step. Our remaining arguments will cover the case of such $A_{u} \vee B_{v}^{\prime}$.

Henceforth, we assume $x_{i_{r_{k}}} \in x\left(A_{u}\right)$ and $x_{i_{r_{k}}} \notin x\left(B_{v}\right)$. Notice that $x\left(A_{u}\right) \vee$ $\left.x\left(B_{v}\right)\right|_{x_{i_{1}} \cdots x_{i_{r_{k}-1}}}$ must be reduced, since otherwise $A_{u} \vee B_{v}$ would have already been collapsed by the series of collapses yielding $\sim^{r_{k}-1}$, by virtue of our collapsing order and our use of induction on length. Thus, we have two cases left to consider, depending whether (a) $\left.\left(x\left(A_{u} \vee B_{v}\right)\right)\right|_{x_{i_{1}} \cdots x_{r_{r_{k}}}}$ is also reduced, or (b) $x_{i_{r_{k}}}$ forms a deletion pair with a letter to its left in $x\left(A_{u} \vee B_{v}\right)$. 
Now to (a), namely the case where $\left.x\left(A_{u} \vee B_{v}\right)\right|_{x_{i_{1}} \cdots x_{i_{r_{k}}}}$ is reduced. The Coxeter group element $w\left(\left.x\left(A_{u}\right)\right|_{x_{i_{1}} \cdots x_{i_{r_{k}}}}\right)=w\left(\left.x\left(B_{v}\right)\right|_{x_{i_{1}} \cdots x_{i_{r_{k}}}}\right)=$ $w\left(\left.x\left(B_{v}\right)\right|_{x_{i_{1}} \cdots x_{r_{r_{k}-1}}}\right)$ must in this case be strictly less than $w\left(\left(A_{u} \vee\right.\right.$ $\left.\left.B_{v}\right)\left.\right|_{x_{1} \cdots x_{i_{k}}}\right)$ in Bruhat order. It follows from this that reading $x\left(A_{u} \vee\right.$ $\left.B_{v}\right)\left.\right|_{x_{1} \cdots x_{i_{r_{k}}}}$ from left to right, we must encounter a leftmost letter $x_{i_{j}}$ whose associated reflection is not one of the associated reflections for $\left.x\left(A_{u}\right)\right|_{x_{1} \cdots x_{i_{k}}}$ and likewise for $\left.x\left(B_{v}\right)\right|_{x_{i_{1}} \cdots x_{i_{k}}}$, hence a letter $x_{i_{j}}$ which may be deleted to obtain a new $x$-expression $\left.x\left(A_{u} \vee B_{v}\right)\right|_{x_{i_{1}} \cdots x_{r_{r_{k}}}} \backslash x_{i_{j}}$ where just the letter $x_{i_{j}}$ has been deleted; this expression will have the property that its associated Coxeter group element is again greater than or equal to both $w\left(\left.x\left(A_{u}\right)\right|_{x_{i_{1}} \cdots x_{i_{r_{k}}}}\right)$ and $w\left(\left.x\left(B_{v}\right)\right|_{x_{i_{1}} \cdots x_{r_{r_{k}}}}\right)$ in Bruhat order. Choose the leftmost such letter $x_{i_{j}}$

If $j \neq r_{k}$, we must therefore have $A_{u} \sim r_{k}-1 A^{\prime}$ for some $A^{\prime}$ such that $x\left(A^{\prime}\right)$ is a subexpression of $x\left(A_{u} \vee B_{v}\right) \backslash x_{i_{j}}$, with $x\left(A^{\prime}\right)$ including $x_{i_{r_{k}}}$, omitting $x_{i_{j}}$, and satisfying $w\left(\left.x\left(A^{\prime}\right)\right|_{x_{i_{1}} \cdots x_{i_{r_{k}}-1}}\right)=w\left(\left.x\left(A_{u}\right)\right|_{x_{i_{1}} \cdots x_{i_{r_{k}}-1}}\right)$, by virtue of Lemma 6.23 together with the definition of Bruhat order as an order based on taking subwords. Similarly, there must exist $B^{\prime}$ with $x\left(B^{\prime}\right)$ also a subexpression of $x\left(A_{u} \vee B_{v}\right) \backslash x_{i_{j}}$ such that $B_{v} \sim^{r_{k}-1} B^{\prime}$ for $x\left(B^{\prime}\right)$ omitting both $x_{i_{j}}$ and $x_{i_{r_{k}}}$, with $w\left(\left.x\left(B_{v}\right)\right|_{x_{i_{1}} \cdots x_{i_{r_{k}-1}}}\right)=w\left(\left.x\left(B^{\prime}\right)\right|_{x_{i_{1}} \cdots x_{i_{r_{k}-1}}}\right)$. Thus, $x\left(A_{u} \vee B_{v}\right) \backslash x_{i_{j}}$ gives an upper bound for $x\left(A_{u}\right)$ and $x\left(B_{v}\right)$ which is strictly contained in $x\left(F^{\prime}\right)=x\left(A_{u} \vee B_{v}\right)$ with $w\left(x\left(A_{u} \vee B_{v}\right)\right) \neq w\left(x\left(A_{u} \vee B_{v}\right) \backslash x_{i_{j}}\right)$, contradicting $F^{\prime}$ being a least upper bound for $A_{u}$ and $B_{v}$.

If $j=r_{k}$, then $l\left(w\left(\left.x\left(A_{u} \vee B_{v}\right)\right|_{x_{i_{1}} \cdots x_{i_{k}}}\right)\right)=l\left(w\left(\left.x\left(A_{u}\right)\right|_{x_{i_{1}} \cdots x_{i_{r_{k}}}}\right)\right)+1=$ $l\left(w\left(\left.x\left(B_{v}\right)\right|_{i_{1} \cdots x_{i_{k}}}\right)\right)+1$ where $l$ denotes Coxeter-theoretic length. But since $\left.x\left(A_{u} \vee B_{v}\right)\right|_{x_{1} \cdots x_{i_{k}}}$ is reduced, we cannot delete from it one letter to obtain $\left.x\left(A_{u}\right)\right|_{x_{1} \cdots x_{i_{k}}}$ and a different individual letter to obtain $\left.x\left(B_{v}\right)\right|_{x_{i_{1}} \cdots x_{i_{r_{k}}}}$, since that would imply $w\left(\left.x\left(A_{u}\right)\right|_{x_{i_{1}} \cdots x_{i_{r_{k}}}}\right) \neq w\left(\left.x\left(B_{v}\right)\right|_{x_{i_{1}} \cdots x_{i_{k}}}\right)$ by the exchange axiom for Coxeter groups, a contradiction. This completes our proof in case (a).

Now to case (b), i.e. the case where $x_{i_{r_{k}}}$ is redundant in $\left.x\left(A_{u} \vee B_{v}\right)\right|_{i_{i_{1}} \cdots x_{i_{r_{k}}}}$ and where $\left.x\left(A_{u} \vee B_{v}\right)\right|_{x_{i_{1}} \cdots x_{i_{r_{k}-1}}}$ is reduced. We then have $A_{u} \vee B_{v} \sim r_{k}-1$ $A \vee B$ unless $w\left(\left.x\left(A_{u} \vee B_{v}\right)\right|_{x_{i_{1}} \cdots x_{i_{r_{k}}-1}}\right) \neq w\left(\left.x(A \vee B)\right|_{x_{i_{1}} \cdots x_{i_{r_{k}}-1}}\right)$, since $x_{i_{r_{k}}}$ appears both in $\left.x(A \vee B)\right|_{x_{i_{1}} \cdots x_{i_{k}}}$ and in $\left.x\left(A_{u} \vee B_{v}\right)\right|_{x_{i_{1}} \cdots x_{i_{k}}}$ and is redundant in both. But $w\left(\left.x(A \vee B)\right|_{x_{i_{1}} \cdots x_{i_{r_{k}}}}\right)$ also equals $w\left(\left.B_{v}\right|_{x_{i_{1}} \cdots x_{i_{r_{k}}-1}}\right)$ which is a subword of $w\left(\left.x\left(A_{u} \vee B_{v}\right)\right|_{x_{i_{1}} \cdots x_{i_{r_{k}-1}}}\right)$, so this implies that $w\left(\left.x(A \vee B)\right|_{x_{i_{1}} \cdots x_{i_{r_{k}}}}\right)$ must be strictly less than $w\left(\left.x\left(A_{u} \vee B_{v}\right)\right|_{x_{i_{1}} \cdots x_{r_{r_{k}-1}}}\right)$ in Bruhat order. But this means there is a letter $x_{i_{j}}$ we may delete from $\left.x\left(A_{u} \vee B_{v}\right)\right|_{x_{i_{1}} \cdots x_{i_{r_{k}-1}}}$ to obtain a word whose associated Coxeter group element is still greater than or equal to $w\left(\left.x\left(B_{v}\right)\right|_{x_{i_{1}} \cdots x_{r_{r_{k}-1}}}\right)$ in Bruhat order, and hence is also greater than or equal 
to $w\left(\left.x\left(A_{u}\right)\right|_{x_{i_{1} \cdots x_{r_{k}-1}}}\right)$ in Bruhat order since

$$
w\left(\left.x\left(A_{u}\right)\right|_{x_{i_{1}} \cdots x_{i_{r_{k}}-1}}\right) \leq \text { Bruhat } w\left(\left.x\left(A_{u}\right)\right|_{x_{i_{1}} \cdots x_{r_{k}}}\right)=w\left(\left.x\left(B_{v}\right)\right|_{x_{i_{1}} \cdots x_{i_{r_{k}}-1}}\right) .
$$

But then deleting this $x_{i_{j}}$ from $x\left(A_{u} \vee B_{v}\right)$ yields an upper bound for $A_{u}$ and $B_{v}$ just prior to the $k$-th collapse whose $x$-expression is strictly contained in $x\left(A_{u} \vee B_{v}\right)$ with distinct associated Coxeter group elements, i.e. with $w\left(\left.x\left(A_{u} \vee B_{v}\right) \backslash x_{i_{j}}\right|_{x_{i_{1}} \cdots x_{i_{r_{k}-1}}}\right) \neq w\left(\left.x\left(A_{u} \vee B_{v}\right)\right|_{x_{i_{1}} \cdots x_{i_{r_{k}}-1}}\right)$. Thus, we get a cell that is an upper bound for $A_{u}$ and $B_{v}$ that is strictly contained in $A_{u} \vee B_{v}$, contradicting $A_{u} \vee B_{v}$ being a least upper bound. This completes case (b).

Next, we give a projection map $\pi_{u}^{v}$ from any closed cell $\bar{\sigma}_{v}$ in $\left(\mathbb{R}_{\geq 0}^{d} \cap\right.$ $\left.S_{1}^{d-1}\right) / \sim$ onto an open cell $\sigma_{u}$ in its boundary, choosing our notation to reflect that $\overline{\sigma_{v}}$ is mapped by $\overline{f_{\left(i_{1}, \ldots, i_{d}\right)}}$ to $Y_{v}$ while $\sigma_{u}$ is mapped by $\overline{f_{\left(i_{1}, \ldots, i_{d}\right)}}$ to $Y_{u}^{o}$. To be more precise, $\pi_{u}^{v}$ applies to the union of open cells contained in $\bar{\sigma}_{v}$ that have $\sigma_{u}$ in their closure. Once equipped with this projection map, we may define the links of cells using the notion of link provided in Definition 4.25 which is based on ideas from stratified Morse theory (cf. [14]). It is most natural to define this projection map using all of $\mathbb{R}_{>0}^{d}$ at once and its quotient spaces $\mathbb{R}_{\geq 0} / \sim$ as well as $\mathbb{R}_{\geq 0} / \sim_{k}$ induced by the collapsing process that we have developed over the course of this section. This is the approach that we take.

Definition 6.31 Consider any $x=\left(t_{1}, \ldots, t_{d}\right)$ in the aforementioned domain of $\pi_{u}^{v}$, choosing the representative for the equivalence class of $x$ under $\sim$ whose only nonzero parameters appear in the positions of letters in the rightmost reduced word for $v$ appearing as a subword of $\left(i_{1}, \ldots, i_{d}\right)$. Let $\left(i_{j_{1}}, \ldots, i_{j_{r}}\right)$ be this subword. The projection map given by $u \subseteq \bar{v}$, denoted $\pi_{u}^{v}$, sets to 0 each $t_{i}$ not appearing in either the rightmost subword of $\left(i_{j_{1}}, \ldots, i_{j_{r}}\right)$ that is a reduced word for $u$ or obtained by reading our word from right to left, including additionally just those letters which are redundant (in the sense of not increasing the 0-Hecke algebra theoretic length) when appended to the word comprised of those letters to its right that have already been chosen.

For intermediate stages in the collapsing process, instead of projecting onto a cell indexed by $u \in W$, we project to a $\sim_{k}$-equivalence class of faces of

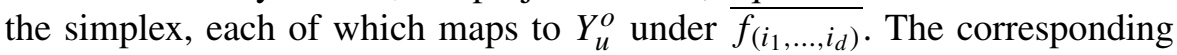
intermediate projection maps are defined completely analogously to $\pi_{u}^{v}$, but now using only those subwords belonging to the allowed equivalence classes mapping under $\overline{f_{\left(i_{1}, \ldots, i_{d}\right)}}$ to cells $Y_{u^{\prime}}^{o}$ with $u \leq u^{\prime} \leq v$. 
We now prove the main theorem of this section, which is largely a matter of pulling together the various lemmas we have proven already. This will require some further notation. Let $\left(i_{1}^{\prime}, \ldots, i_{d^{\prime}}^{\prime}\right)$ be the subword of $\left(i_{1}, \ldots, i_{d}\right)$ associated to the maximal cell $F_{k}$ to be collapsed at the $k$-th collapsing step, so $d^{\prime}$ is the wordlength of $x\left(F_{k}\right)$. Let $\left(j_{1}^{\prime}, \ldots, j_{d^{\prime}}^{\prime}\right)$ be the word obtained from $\left(i_{1}^{\prime}, \ldots, i_{d^{\prime}}^{\prime}\right)$ by applying the series of braid moves giving rise to the change of coordinates homeomorphism $c h$, i.e. the chosen series of (long and short) braid moves applied to $\left(i_{1}^{\prime}, \ldots, i_{d^{\prime}}^{\prime}\right)$ yielding a stutter between positions $r-1$ and $r$ to be used to induce our collapse of $F_{k}$. Note that there must be some $r$ such that $\left(i_{r}^{\prime}, \ldots, i_{d^{\prime}}^{\prime}\right)=\left(i_{r_{k}}, \ldots, i_{d}\right)=\left(j_{r}^{\prime}, \ldots, j_{d^{\prime}}^{\prime}\right)$. The restriction of $\sim_{\left(i_{1}, \ldots, i_{d}\right)}^{r_{k}-1}$ to $\bar{F}_{k}$ equals the equivalence relation $\sim_{\left(i_{1}^{\prime}, \ldots, i_{d^{\prime}}^{\prime}\right)}^{r-1}$. This also carries out exactly the same identifications as $\underset{\left(j_{1}^{\prime}, \ldots, j_{d^{\prime}}^{\prime}\right)}{\sim^{\prime}-1}$ after suitable change of coordinates.

Theorem 6.32 $\left(\mathbb{R}_{\geq 0}^{d} \cap S_{1}^{d-1}\right) / \sim$ is a regular $C W$ complex homeomorphic to a ball. Moreover, the link of any cell is also a regular CW complex homeomorphic to a ball.

Proof We start our proof with the simplex $K_{0}=\mathbb{R}_{\geq 0}^{d} \cap S_{1}^{d-1}$. We perform on $K_{0}$ a series of collapses, using Theorem 4.21 and its generalization described in Corollary 4.24 to justify that each of these collapses preserves homeomorphism type as well as the property of having a regular CW complex. These collapses use the families of curves introduced in Definition 6.18. We assume inductively that all earlier collapses were performed successfully in order to justify that the $k$-th collapse preserves homeomorphism type, regularity, and all the requisite properties for our inductive step. We also assume inductively all results in the paper for all strictly smaller $d$.

We use the cell collapsing order given just after Example 6.17. The $k$-th collapsing step is specified by a deletion pair $\left\{x_{i_{l_{k}}}, x_{i_{r_{k}}}\right\}$ in an $x$ expression $x\left(F_{k}\right)$, with the cell $\bar{F}_{k} / \sim_{k} \in\left(\mathbb{R}_{\geq 0}^{d} \cap S_{1}^{d-1}\right) / \sim_{k}$ collapsed across curves each having one endpoint in the closed cell $\bar{G}_{1} / \sim_{k}$ which consists of the points of $\overline{F_{k}} / \sim_{k}$ with $t_{l_{k}}=0$ and the other endpoint in the closed cell $\bar{G}_{2} / \sim_{k}$ instead having $t_{r_{k}}=0$. The series of lemmas we have just proven will allow us to verify all the requirements for our curves to be a parallel-like family of curves (in the extended sense of Corollary 4.24) and to check all of the hypotheses of Theorem 4.21 (again as extended in Corollary 4.24), once we show how to incorporate the requisite change of coordinates homeomorphisms $c h$ into the picture. This change of coordinates which will help us accomplish the $k$-th collapse will be done on $\left(\mathbb{R}_{\geq 0}^{d} \cap S_{1}^{d-1}\right) / \sim^{r_{k}-1}$ to create a stutter, so in particular will be done prior to the $k$-th collapse.

The fact that $i_{r_{k}}=i_{r}^{\prime}$ is the leftmost right endpoint of a deletion pair in $x\left(F_{k}\right)$ implies that $\left(i_{1}^{\prime}, \ldots, i_{r-1}^{\prime}\right)$ is reduced and hence that $\left(j_{1}^{\prime}, \ldots, j_{r-1}^{\prime}\right)$ is 
also reduced with $w\left(i_{1}^{\prime}, \ldots, i_{r-1}^{\prime}\right)=w\left(j_{1}^{\prime}, \ldots, j_{r-1}^{\prime}\right)$ and hence $w\left(i_{1}^{\prime}, \ldots, i_{d^{\prime}}^{\prime}\right)$ $=w\left(j_{1}^{\prime}, \ldots, j_{d^{\prime}}^{\prime}\right)$. Let us also now choose a reduced word $\left(j_{1}, \ldots, j_{d}\right)$ such that $j_{i}=j_{i}^{\prime}$ for all $i \leq d^{\prime}$. The point of extending $\left(j_{1}^{\prime}, \ldots, j_{d^{\prime}}^{\prime}\right)$ to this longer reduced word $\left(j_{1}, \ldots, j_{d}\right)$ is to have at our disposal a regular $\mathrm{CW}$ ball $\left(\mathbb{R}_{\geq 0}^{d} \cap S_{1}^{d-1}\right) / \sim_{\left(j_{1}, \ldots, j_{d}\right)}^{r-1}$ given by $\left(j_{1}, \ldots, j_{d}\right)$ with this ball of the correct dimension so as to be homeomorphic to the complex $\left(\mathbb{R}_{\geq 0}^{d} \cap S_{1}^{d-1}\right) / \sim_{m}$ given by $\left(i_{1}, \ldots, i_{d}\right)$ for each $m>0$ and which for $m \geq \bar{k}$ will also have $\left(\mathbb{R}_{\geq 0}^{d^{\prime}} \cap S_{1}^{d^{\prime}-1}\right) / \sim_{\left(j_{1}^{\prime}, \ldots, j_{d^{\prime}}^{\prime}\right)}$ as a subcomplex.

The image under $c h$ of each curve in our family covering $\bar{F}_{k} / \sim_{\left(i_{1}^{\prime}, \ldots, i_{d^{\prime}}\right)}^{r-1}$ will be a collection of points $\left(t_{1}^{\prime}, \ldots, t_{d}^{\prime}\right)$ with $t_{r_{k}-1}^{\prime}+t_{r_{k}}^{\prime}$ held constant and each $t_{i}^{\prime}$ for $i \notin\left\{r_{k}-1, r_{k}\right\}$ also held constant, all within $\left(\mathbb{R}_{\geq 0}^{d^{\prime}} \cap\right.$ $\left.S_{1}^{d^{\prime}-1}\right) / \sim_{\left(i_{1}^{\prime}, \ldots, i_{d^{\prime}}^{\prime}\right)}^{r-1}$. This will imply that $c h$ maps our family of curves to the images under a series of collapsing maps of a family of parallel line segments covering the cell having the structure $\left(\mathbb{R}_{\geq 0}^{d^{\prime}} \cap S_{1}^{d^{\prime}-1}\right) / \sim_{\left(j_{1}^{\prime}, \ldots, j_{d^{\prime}}^{\prime}\right.}^{r-1}$ within the regular CW complex $\left(\mathbb{R}_{\geq 0}^{d} \cap S_{1}^{d-1}\right) / \sim_{\left(j_{1}, \ldots, j_{d}\right)}^{r-1}$. In particular, $c h^{-1}$ also thereby will induce a transfer of a parametrization function to each nontrivial curve in the closed cell $\bar{F}_{k} / \sim_{\left(i_{1}, \ldots, i_{d}\right)}^{r_{k}-1}$ in $\left(\mathbb{R}_{\geq 0}^{d} \cap S_{1}^{d-1}\right) / \sim_{\left(i_{1}, \ldots, i_{d}\right)}^{r_{k}-1}$ which is a continuous function to $[0,1]$ on the union of these nontrivial curves. Moreover, condition (DE) together with the fact that collapses subsequent to those producing $\sim^{r_{k}-1}$ and leading to $\sim_{k}$ restrict to homeomorphisms on each closed cell that they do not collapse will imply that these collapses will also carry forward these curve parametrizations for all curves that stay nontrivial under the intermediate collapses to the quotient complex $\left(\mathbb{R}_{\geq 0}^{d} \cap S_{1}^{d-1}\right) / \sim_{k}$ from the quotient complex $\left(\mathbb{R}_{\geq 0}^{d} \cap S_{1}^{d-1}\right) / \sim_{k}^{r_{k}-1}$. Thus, our parallel-like curves for $\bar{F}_{k} / \sim_{k}$ will be the images under $c h^{-1}$ of these parallel-like curves from $\left(\mathbb{R}_{\geq 0}^{d} \cap S_{1}^{d-1}\right) / \sim_{\left(j_{1}, \ldots, j_{d}\right)}^{r-1}$, pushed forward by the collapses yielding $\sim_{k}$ from $\sim_{\left(i_{1}, \ldots, i_{d}\right)}^{r_{k}-1}$. Now let us carefully define the change of coordinates map $c h$ (and thereby $c h^{-1}$ ).

This map $c h$ is most naturally defined on the closed cell $\bar{F}_{k} / \sim_{\left(i_{1}, \ldots, i_{d}\right)}^{r_{k}-1}$. Lemma 6.24 (as explained in Remark 6.26) proves $c h$ is a cell structure preserving homeomorphism from $\bar{F}_{k} / \sim_{\left(i_{1}^{\prime}, \ldots, i_{d^{\prime}}\right)}^{r-1}=\bar{F}_{k} / \sim_{\left(i_{1}, \ldots, i_{d}\right)}^{r_{k}-1}$ to $\left(\mathbb{R}_{\geq 0}^{d^{\prime}} \cap S_{1}^{d^{\prime}}\right) / \sim_{\left(j_{1}^{\prime}, \ldots, j_{d^{\prime}}^{\prime}\right)}^{r-1}$. Let us now show how $c h$ (or equivalently $c h^{-1}$ ) may be extended to a homeomorphism from the entire quotient complex $\left(\mathbb{R}_{\geq 0}^{d} \cap S_{1}^{d-1}\right) / \sim_{\left(i_{1}, \ldots, i_{d}\right)}^{r_{k}-1}$ to the quotient complex $\left(\mathbb{R}_{\geq 0}^{d} \cap S_{1}^{d-1}\right) / \sim_{\left(j_{1}, \ldots, j_{d}\right)}^{r_{k}-1}$ given by the word $\left(j_{1}, \ldots, j_{d}\right)$. It will be a necessity for our upcoming approach to collapsing $\bar{F}_{k} / \sim_{k}$ via transfer of parallel-like curves carried out by the map $c h^{-1}$ that $f_{\left(i_{1}^{\prime}, \ldots, i_{d^{\prime}}^{\prime}\right)}(x)=f_{\left(j_{1}^{\prime}, \ldots, j_{d^{\prime}}^{\prime}\right)}(c h(x))$ for each $x \in$ 
$\bar{F}_{k} / \sim_{\left(i_{1}, \ldots, i_{d}\right)}^{r_{k}-1}$; however, this relationship between $x$ and $\operatorname{ch}(x)$ will not be needed for the extension of $c h^{-1}$ to points $x$ outside of $\bar{F}_{k} / \sim_{k}$, because the collapsing map we will use to collapse $F_{k}$ will restrict to an injection outside of $\bar{F}_{k} / \sim_{k}$. Thus, we may simply extend $c h^{-1}$ to a neighborhood $N$ of $\bar{F}_{k} / \sim_{k}$ by thickening the boundary of $\bar{F}_{k} / \sim_{k}$ to $\partial\left(\bar{F}_{k}\right) / \sim_{k} \times[0, \epsilon)$ and letting $c h^{-1}(x, t)=\left(c h^{-1}(x), t\right)$ for each $t \in[0, \epsilon)$. This thickening is possible since the closed complement of $\bar{F}_{k}$ within the boundary of a cell of dimension one higher is a topological manifold with boundary, by Lemma 4.21 applied to the earlier collapse, hence has a collar by Theorem 2.8 .

It is worth emphasizing that the transfer of curve parametrizations resulting from $c h^{-1}$ will not in any way actually modify the collapsing procedure that we already indicated we would use and which has been described and analyzed in detail earlier in this section of the paper. Rather, the map $c h^{-1}$ on $\bar{F}_{k}$ is used to justify that we indeed have parallel-like curves in a suitable sense (i.e. as in Corollary 4.24) to enable the collapse of $F_{k}$ even when long braid moves are needed to create a stutter in $x\left(F_{k}\right)$; we do this by giving an alternate way that we could have obtained the closed cell $\overline{F_{k}} / \sim_{\left(i_{1}^{\prime}, \ldots, i_{d^{\prime}}\right)}^{r-1}$. This in turn gives an alternate way we could have obtained the closed cell $\overline{F_{k}} / \sim_{k}$ by performing exactly the collapses on $\left(\mathbb{R}_{\geq 0}^{d} \cap S_{1}^{d-1}\right) / \sim_{\left(i_{1}, \ldots, i_{d}\right)}^{r_{k}-1}$ (and in the process also on $\overline{F_{k}} / \sim_{\left(i_{1}^{\prime}, \ldots, i_{d^{\prime}}^{\prime}\right)}^{r-1}$ that we had planned for word $\left(i_{1}, \ldots, i_{d}\right)$ to get from the equivalence relation $\sim^{r_{k}-1}$ to the equivalence relation $\sim_{k}$, but having incorporated the transfer map $c h^{-1}$ at the step where we had equivalence relation $\sim^{r_{k}-1}$. In other words, we regard $\left(\mathbb{R}_{\geq 0}^{d} \cap S_{1}^{d-1}\right) / \sim_{\left(i_{1}, \ldots, i_{d}\right)}^{r_{k}-1}$ as our starting point complex $K_{0}$ for purpose of justifying the collapse of $\bar{F}_{k} / \sim_{k}$.

Having now realized our curves from Definition 6.18 as the images of parallel line segments in a suitable sense, i.e. with a transfer from one complex to another complex potentially involved, we now turn to the remaining requirements for parallel-like curves. Lemmas 6.29, 6.30 and 4.19 confirm that the requirements (DIP), (DE) (as defined in Conditions 4.9 and 4.11, respectively) and condition (LUB) hold for the family of curves to be collapsed in the $k$-th collapse just prior to this collapse, assuming all earlier collapses were done successfully. Thus, all of the requirements of Theorem 4.21 (or at least their relaxations as in Corollary 4.24) are met, enabling us to repeatedly collapse cells until all cells given by non-reduced subwords of $\left(i_{1}, \ldots, i_{d}\right)$ have been eliminated, preserving homeomorphism type and regularity at each step. Thus, the end result is a regular CW complex homeomorphic to a ball. Regularity and homeomorphism type for links, as defined in Definition 4.25, follow from Lemma 6.27, since it allows us to invoke Lemma 4.26, noting that the transversality requirements follow easily from the definition of our series of projection maps. 


\subsection{Regularity and homeomorphism type of $Y_{w}$}

We now finally turn to studying the topological structure of $Y_{w}$ itself. We will use the fact that $\left(\mathbb{R}_{\geq 0}^{d} \cap S_{1}^{d-1}\right) / \sim$ is a regular CW complex homeomorphic to a ball to prove now that $Y_{w}$ is as well. First we must verify Condition 4 of Theorem 1.3 for the characteristic maps $\overline{f_{\left(i_{1}, \ldots, i_{d}\right)}}:\left(\mathbb{R}_{\geq 0}^{d} \cap S_{1}^{d-1}\right) / \sim \rightarrow Y_{w}$ and their restrictions to the closed cells of $\left(\mathbb{R}_{\geq 0}^{d} \cap S_{1}^{d-1}\right) / \sim$. This is done in Lemma 6.33 below, which will only require the following properties of $\sim$, which are immediate from the definition of $\sim$ :

(1) Each $p \in \partial\left(\mathbb{R}_{\geq 0}^{d} \cap S_{1}^{d-1}\right)$ whose $x$-expression is not reduced is identified by $\sim$ with a point having more parameters set to 0

(2) $p \sim q$ implies $w\left(f_{\left(i_{1}, \ldots, i_{d}\right)}(p)\right)=w\left(f_{\left(i_{1}, \ldots, i_{d}\right)}(q)\right)$.

The points in a cell boundary, i.e. the preimage of one of the attaching maps, are obtained by setting some positive parameters to 0 .

Lemma 6.33 Given a reduced word $\left(i_{1}^{\prime}, \ldots, i_{d^{\prime}}^{\prime}\right)$ which is a subword of reduced word $\left(i_{1}, \ldots, i_{d}\right)$, then $\overline{f_{\left(i_{1}, \ldots, i_{d}\right)}}$ restricted to the codimension one faces of $F=\overline{R_{\left\{i_{1}^{\prime}, \ldots, i_{d^{\prime}}^{\prime}\right\}}} / \sim$ is an injection into $Y_{s_{i_{1}^{\prime}} \ldots s_{i_{d^{\prime}}^{\prime}}}$.

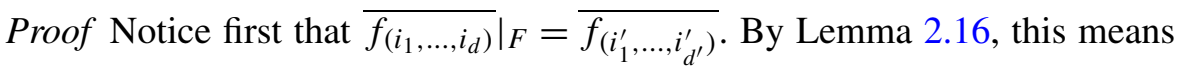
that $w\left(x_{i_{1}^{\prime}} \cdots \hat{x}_{i_{r}^{\prime}} \cdots x_{i_{d^{\prime}}^{\prime}}\right) \neq w\left(x_{i_{1}^{\prime}} \cdots \hat{x}_{i_{r^{\prime}}} \cdots x_{i_{d^{\prime}}^{\prime}}\right)$ for $r \neq r^{\prime}$, provided that both expressions are reduced, since that implies that the map $w$ just replaces each $x_{i}$ by simple reflection $s_{i}$. Consequently, boundary points obtained by sending distinct single parameters to 0 to obtain reduced expressions of length one shorter must belong to distinct cells, hence have distinct images under $\overline{f_{\left(i_{1}^{\prime}, \ldots, i_{d^{\prime}}^{\prime}\right)}}$. On the other hand, varying values of the nonzero parameters while keeping fixed which parameters are 0 and which are nonzero with the subexpression of nonzero parameters a reduced expression must also yield points with distinct images under $\overline{f_{\left(i_{1}^{\prime}, \ldots, i_{d^{\prime}}^{\prime}\right)}}$, since Lusztig proved that $f_{\left(i_{1}^{\prime}, \ldots, i_{d^{\prime}}^{\prime}\right)}$ acts homeomorphically on $\mathbb{R}_{>0}^{s}$ for $\left(i_{1}^{\prime}, \ldots, i_{d^{\prime}}^{\prime}\right)$ reduced. Combining yields that $\overline{f_{\left(i_{1}^{\prime}, \ldots, i_{d^{\prime}}^{\prime}\right)}}$ is injective upon restriction to the codimension one cells, as desired.

Now to our main result, Theorem 6.34. It is phrased in a somewhat technical way so as to enable proof by induction on the length $d$, and also to overcome the challenge that it was not known previously even that $\overline{f_{\left(i_{1}, \ldots, i_{d}\right)}}\left(\left(\mathbb{R}_{\geq 0}^{d} \cap S_{1}^{d-1}\right) / \sim\right)$ was a CW complex. Theorem 6.34 is immediately followed by corollaries with more natural statements.

To use Theorem 1.3 in proving Theorem 6.34, we will need the preimages of the various characteristic maps to be closed cells in $\left(\mathbb{R}_{\geq 0}^{d} \cap S_{1}^{d-1}\right) / \sim$, since 
this will give condition 5 of our regularity criterion. It is not clear that taking the closure in $\left(\mathbb{R}_{\geq 0}^{d} \cap S_{1}^{d-1}\right) / \sim$ of an open cell which is sent by $\overline{f_{\left(i_{1}, \ldots, i_{d}\right)}}$ to $Y_{\sigma}$ for a Coxeter group element $\sigma$ of length $d^{\prime}<d$ is the same as constructing a complex $\left(\mathbb{R}_{\geq 0}^{d^{\prime}} \cap S_{1}^{d^{\prime}-1}\right) / \sim$ for $\sigma$ itself directly; we overcome this issue by allowing flexibility in the choice of $\sim$ in the statement of the next theorem, in particular allowing the collapsing maps for the closure of a cell which is not top-dimensional to be induced from the collapsing maps on the entire complex.

Theorem 6.34 Let $\left(i_{1}, \ldots, i_{d}\right)$ be a reduced word for $w \in W$. Let $\sim$ be the identifications given by any series of face collapses (cf. Definition 4.1) on $\mathbb{R}_{>0}^{d} \cap S_{1}^{d-1}$ such that $(1) x \sim y$ implies $f_{\left(i_{1}, \ldots, i_{d}\right)}(x)=f_{\left(i_{1}, \ldots, i_{d}\right)}(y)$, and (2) the series of collapses eliminates all regions whose words are not reduced. Then $\overline{f_{\left(i_{1}, \ldots, i_{d}\right)}}:\left(\mathbb{R}_{\geq 0}^{d} \cap S_{1}^{d-1}\right) / \sim \rightarrow Y_{w}$ is a homeomorphism which preserves cell structure.

Proof The proof is by induction on $d$, with the case $d=1$ being trivial. Therefore, we may assume the result for all finite Coxeter group elements of length strictly less than $d$. Remark 2.5 enables us to deduce continuity of $\overline{f_{\left(i_{1}, \ldots, i_{d}\right)}}$ from continuity of $f_{\left(i_{1}, \ldots, i_{d}\right)}$. Notice that $f_{\left(i_{1}, \ldots, i_{d}\right)}$ restricts to any region obtained by setting some $t_{i}$ 's to 0 since $x_{i}(0)$ is the identity matrix. Whenever the resulting subword is reduced, results in [20] guarantee that $f_{\left(i_{1}, \ldots, i_{d}\right)}$ acts homeomorphically on this open cell. The requirements of Corollary 3.9 regarding closures of cells in the $(d-1)$-skeleton follow from our inductive hypothesis, along with the fact that any series of face collapses will restrict to one on the closure of any cell. Thus, we may apply Corollary 3.9 to deduce that $\overline{f_{\left(i_{1}, \ldots, i_{d}\right)}}\left(\left(\mathbb{R}_{\geq 0}^{d} \cap S_{1}^{d-1}\right) / \sim\right)$ is a finite CW complex with the restrictions of $\overline{f_{\left(i_{1}, \ldots, i_{d}\right)}}$ to the various cell closures in $\left(\mathbb{R}_{\geq 0}^{d} \cap S_{1}^{d-1}\right) / \sim$ giving the characteristic maps, and that this $\mathrm{CW}$ complex structure satisfies conditions 1,2 and 5 of Theorem 1.3. Lemma 6.33 confirmed condition 4 of Theorem 1.3, while the result of [6] that Bruhat order is shellable and thin gives condition 3. Thus, by Theorem $1.3, \overline{f_{\left(i_{1}, \ldots, i_{d}\right)}}\left(\left(\mathbb{R}_{\geq 0}^{d} \cap S_{1}^{d-1}\right) / \sim\right)$ is a regular CW complex with characteristic maps given by the restrictions of $\overline{f_{\left(i_{1}, \ldots, i_{d}\right)}}$ to the various cell closures, which is exactly what is needed.

Corollary 6.35 If $\left(i_{1}, \ldots, i_{d}\right)$ is a reduced word for $w$, then $f_{\left(i_{1}, \ldots, i_{d}\right)}$ induces a homeomorphism $\overline{f_{\left(i_{1}, \ldots, i_{d}\right)}}:\left(\mathbb{R}_{\geq 0}^{d} \cap S_{1}^{d-1}\right) / \sim \rightarrow Y_{w}$ which preserves cell structure. Hence, $Y_{w}$ is a regular $C W$ complex homeomorphic to a ball with Bruhat interval $(1, w]$ as its closure poset.

Proof By Theorem 6.32, $K=\left(\mathbb{R}_{\geq 0}^{d} \cap S_{1}^{d-1}\right) / \sim$ is a regular CW complex homeomorphic to a ball. We chose $\sim$ so that $f_{\left(i_{1}, \ldots, i_{d}\right)}(x)=f_{\left(i_{1}, \ldots, i_{d}\right)}(y)$ 
whenever $x \sim y$. Combining with Lusztig's result that $f_{\left(i_{1}, \ldots, i_{d}\right)}$ is continuous on $\mathbb{R}_{>0}^{d} \cap S_{1}^{d-1}$ and a homeomorphism on $\mathbb{R}_{>0}^{d} \cap S_{1}^{d-1}$ (see [20], Sect. 4]), as well as the fact that our collapsing maps are identification maps, it follows that $\overline{f_{\left(i_{1}, \ldots, i_{d}\right)}}$ is also continuous on $K$. In particular, $K$ meets all the requirements of Theorem 6.34 .

Corollary 6.36 For $\left(t_{1}, \ldots, t_{d}\right),\left(t_{1}^{\prime}, \ldots, t_{d}^{\prime}\right) \in \mathbb{R}_{\geq 0}^{d}$ and $\left(i_{1}, \ldots, i_{d}\right)$ any reduced word,

$$
x_{i_{1}}\left(t_{1}\right) \cdots x_{i_{d}}\left(t_{d}\right)=x_{i_{1}}\left(t_{1}^{\prime}\right) \cdots x_{i_{d}}\left(t_{d}^{\prime}\right) \quad \text { iff }\left(t_{1}, \ldots, t_{d}\right) \sim\left(t_{1}^{\prime}, \ldots, t_{d}^{\prime}\right) .
$$

Finally we consider $l k(u, w)$. Theorem 6.32 proved regularity and determined homeomorphism type for the links of the cells in $\left(\mathbb{R}_{\geq 0}^{d} \cap S_{1}^{d-1}\right) / \sim$, using the natural projection map in that context (cf. Definition 6.31). This immediately transfers to yield analogous results for links in $Y_{w}$ via our cell-

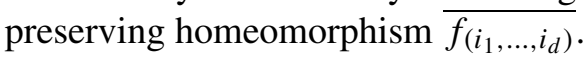

Corollary 6.37 For each $u<w$ in Bruhat order, the subcomplex $l k(u, w)$ of $Y_{w}$ obtained as the image under the cell-preserving homeomorphism $\overline{f_{\left(i_{1}, \ldots, i_{d}\right)}}$ of the corresponding link within $\mathbb{R}_{\geq 0}^{d} / \sim$ is a regular $C W$ complex homeomorphic to a ball having the Bruhat interval $(u, w]$ as its poset of closure relations.

We expect that this notion of link should coincide, at least up to homeomorphism, with the notion of link given by Fomin and Shapiro for $Y_{w}$ in [11] by virtue of Thom's first isotopy lemma (cf. [14]).

It would be interesting to understand better how $\operatorname{lk}(u, w)$ relates both to subword complexes (cf. [17-19]) and also to the synthetic CW complexes for Bruhat intervals studied by Reading in [27].

Acknowledgements The author was funded by NSF grants DMS-0500638, DMS-0757935, DMS-1002636 and DMS-1200730 as well as the Ruth I. Michler Prize of the Association for Women in Mathematics, the last of which provided a research semester at Cornell. She thanks the Cornell mathematics department for its warm hospitality and intellectually stimulating atmosphere during her stay there.

The author is grateful to Sara Billey, Anders Björner, Jim Davis, Sergey Fomin, Mark Goresky, Mike Hopkins, Nets Hawk Katz, Allen Knutson, Chuck Livingston, Mark McConnell, Sergey Melikhov, Ezra Miller, Nathan Reading, David Speyer and Lauren Williams for very helpful discussions and comments on various versions of the paper. She also thanks the anonymous referees for insightful questions and comments which led to substantial improvements in the paper.

Open Access This article is distributed under the terms of the Creative Commons Attribution License which permits any use, distribution, and reproduction in any medium, provided the original author(s) and the source are credited. 


\section{References}

1. Armstrong, D., Hersh, P.: Sorting orders, subword complexes, Bruhat order and total positivity. Adv. Appl. Math. 46, 46-53 (2011). (Special volume in honor of the 60th birthday of Dennis Stanton)

2. Berenstein, A., Zelevinsky, A.: Total positivity in Schubert varieties. Comment. Math. Helv. 72, 128-166 (1997). CMP 97:14

3. Bing, R.H.: A homeomorphism between the 3-sphere and the sum of two solid horned spheres. Ann. Math. (2) 56, 354-362 (1952)

4. Björner, A.: Posets, regular CW complexes and Bruhat order. Eur. J. Comb. 5(1), 7-16 (1984)

5. Björner, A., Brenti, F.: Combinatorics of Coxeter Groups. Graduate Texts in Mathematics, vol. 231. Springer, Berlin (2005)

6. Björner, A., Wachs, M.: Bruhat order of Coxeter groups and shellability. Adv. Math. 43(1), 87-100 (1982)

7. Bredon, G.: Topology and Geometry. Graduate Texts in Mathematics, vol. 139. Springer, Berlin (1993)

8. Connelly, R.: A new proof of Brown's collaring theorem. Proc. Amer. Math. Soc. 27, 180-182 (1971)

9. Danaraj, G., Klee, V.: Shellings of spheres and polytopes. Duke Math. J. 41, 443-451 (1974)

10. Fomin, S., Greene, C.: Noncommutative Schur functions and their applications. Discrete Math. 193, 179-200 (1998). (Special issue in honor of Adriano Garsia)

11. Fomin, S., Shapiro, M.: Stratified spaces formed by totally positive varieties. Dedicated to William Fulton on the occasion of his 60th birthday. Mich. Math. J. 48, 253-270 (2000)

12. Fomin, S., Stanley, R.: Schubert polynomials and the NilCoxeter algebra. Adv. Math. 103(2), 196-207 (1994)

13. Fomin, S., Zelevinsky, A.: Double Bruhat cells and total positivity. J. Am. Math. Soc. 12(2), 335-380 (1999)

14. Goresky, M., MacPherson, R.: Stratified Morse Theory. Ergebnisse der Mathematik und ihrer Grenzgebiete (3), vol. 14. Springer, Berlin (1988). xiv + 272 pp.

15. Humphreys, J.: Linear Algebraic Groups. Graduate Texts in Mathematics, vol. 21. Springer, Berlin (1975)

16. Humphreys, J.: Reflection Groups and Coxeter Groups. Cambridge Studies in Advanced Mathematics, vol. 29. Cambridge University Press, Cambridge (1990)

17. Knutson, A.: Schubert patches degenerate to subword complexes. Transform. Groups 13(3-4), 715-726 (2008)

18. Knutson, A., Miller, E.: Subword complexes in Coxeter groups. Adv. Math. 184(1), 161176 (2004)

19. Knutson, A., Miller, E.: Gröbner geometry of Schubert polynomials. Ann. Math. (2) 161(3), 1245-1318 (2005)

20. Lusztig, G.: Total positivity in reductive groups. In: Lie Theory and Geometry: in Honor of Bertram Kostant. Progress in Mathematics, vol. 123. Birkhäuser, Basel (1994)

21. Lusztig, G.: Introduction to total positivity. In: Positivity in Lie Theory: Open Problems. de Gruyter Exp. Math., vol. 26, pp. 133-145. de Gruyter, Berlin (1998)

22. Lusztig, G.: Total positivity in partial flag manifolds. Represent. Theory 2, 212-242 (2004)

23. Macdonald, I.G.: Notes on Schubert Polynomials. Publications du Laboratoire de Combinatoire et d'Informatique Mathematique, vol. 6. LACIM, Montreal (1991)

24. Munkres, J.: Elements of Algebraic Topology. Addison-Wesley, Reading (1984)

25. Postnikov, A.: Total positivity, Grassmannians, and networks. arXiv:math.CO/0609184

26. Postnikov, A., Speyer, D., Williams, L.: Matching polytopes, toric geometry and the nonnegative part of the Grassmannian. J. Algebr. Comb. 30(2), 173-191 (2009) 
27. Reading, N.: The cd-index of Bruhat intervals. Electron. J. Comb. 11(1), 74 (2004), 25 $\mathrm{pp}$.

28. Rietsch, K.: Closure relations for totally nonnegative cells in $G / P$. Math. Res. Lett. 13(56), 775-786 (2006)

29. Rietsch, K., Williams, L.: The totally nonnegative part of $G / P$ is a $C W$ complex. Transform. Groups 13, 839-853 (2008). (Special issue for Kostant's birthday)

30. Rietsch, K., Williams, L.: Discrete Morse theory for totally nonnegative flag varieties. Adv. Math. 223(6), 1855-1884 (2010)

31. Rourke, C.P., Sanderson, B.J.: Introduction to Piecewise-Linear Topology. Ergebnisse der Mathematik und ihrer Grenzgebiete, vol. 69. Springer, Berlin (1972)

32. Spanier, E.: Algebraic Topology. Springer, Berlin (1966)

33. Stanley, R.: Enumerative Combinatorics, vol. 1, 2nd edn. Cambridge University Press, Cambridge (2012)

34. Vick, J.: Homology Theory: An Introduction to Algebraic Topology. Springer, Berlin (1994)

35. Williams, L.: Shelling totally nonnegative flag varieties. J. Reine Angew. Math. 2007(609), $1-22(2007)$ 